San Jose State University

SJSU ScholarWorks

Master's Theses

Master's Theses and Graduate Research

Fall 2013

\title{
Real-time Auto Tuning of a Closed Loop Second Order System with Internal Time Delay Using Pseudo Random Binary Sequences
}

David Adams

San Jose State University

Follow this and additional works at: https://scholarworks.sjsu.edu/etd_theses

\section{Recommended Citation}

Adams, David, "Real-time Auto Tuning of a Closed Loop Second Order System with Internal Time Delay Using Pseudo Random Binary Sequences" (2013). Master's Theses. 4371.

DOI: https://doi.org/10.31979/etd.x2vp-ab9a

https://scholarworks.sjsu.edu/etd_theses/4371

This Thesis is brought to you for free and open access by the Master's Theses and Graduate Research at SJSU ScholarWorks. It has been accepted for inclusion in Master's Theses by an authorized administrator of SJSU ScholarWorks. For more information, please contact scholarworks@sjsu.edu. 
REAL-TIME AUTO TUNING OF A CLOSED-LOOP SECOND-ORDER SYSTEM WITH INTERNAL TIME-DELAY USING PSEUDO-RANDOM BINARY SEQUENCES

\author{
A Thesis \\ Presented to \\ The Faculty of the Department of Electrical Engineering \\ San José State University
}

\author{
In Partial Fulfillment \\ of the Requirements for the Degree \\ Master of Science
}

by

David M. Adams

December 2013 
(C) 2013

David M. Adams

ALL RIGHTS RESERVED 
The Designated Thesis Committee Approves the Thesis Titled

\author{
REAL-TIME AUTO TUNING OF A CLOSED-LOOP SECOND-ORDER SYSTEM WITH \\ INTERNAL TIME-DELAY USING PSEUDO-RANDOM BINARY SEQUENCES \\ by \\ David M. Adams
}

\title{
APPROVED FOR THE DEPARTMENT OF ELECTRICAL ENGINEERING SAN JOSÉ STATE UNIVERSITY
}

December 2013

Dr. Peter Reischl Department of Electrical Engineering

Dr. Ping Hsu Department of Electrical Engineering

Dr. Vitaly Spitsa Department of Electrical Engineering 


\begin{abstract}
REAL-TIME AUTO TUNING OF A CLOSED-LOOP SECOND-ORDER SYSTEM WITH INTERNAL TIME-DELAY USING PSEUDO-RANDOM BINARY SEQUENCES

by David M. Adams

This research yielded a real-time auto tuning algorithm to adaptively tune a proportional integral and derivative (PID) controller for a first or second-order system with internal time-delay. The method uses a 15-bit pseudo-random binary sequence as an input to obtain the closed-loop system impulse response while the system is operating. Time-delay is assessed by analysis of the estimated closed-loop impulse response and is used in the system model for closed-loop pole assessment. The fast fourier transform of the estimated impulse response produces an estimate of the frequency response data, and a non-linear regression optimization technique, utilizing MATLAB, identifies the closed-loop system transfer function based on assumed form. Closed-loop poles are then placed, based on an iterative tuning study, automatically by the algorithm to achieve a user-defined overshoot and ensure stability of the system with time-delay. This is accomplished by adjusting the PID compensator gains. The algorithm is capable of tuning the system from an initially stable set of PID gains to within 5\% of the user-defined overshoot. The research demonstrates that the auto tuning method is feasible for time-delays on the order of the plant time constant but is extendable to larger time-delays.
\end{abstract}




\section{ACKNOWLEDGEMENTS}

This thesis was produced with the aid and support of many individuals that cannot all be named. Without the support of dedicated faculty and unending support from friends and family, this thesis would never have been complete. I would first like to thank my advisor and mentor Dr. Peter Reischl for his inspirational guidance and support through the many difficulties encountered during this research. I would like to thank Dr. Ping Hsu and Dr. Vitaly Spitsa for providing excellent feedback and industry perspective which helped drive the direction of the work. I would also like to thank my family and friends for their unending support and prayers which motivated me to put my best work forward and allow for the completion of this work. This thesis is dedicated to my loving wife Emily and my firstborn son Nathan who sacrificed so much to give me the opportunity to continue my education. 


\section{TABLE OF CONTENTS}

1 Introduction 1

1.1 A Modern Perspective on Time-Delayed Systems 1

1.2 Traditional Methods for Dealing With Time-Delayed Systems 4

$\begin{array}{lll}1.3 & \text { Motivation for Algorithm Development } & 6\end{array}$

2 Real-Time Auto Tuning Algorithm Development 9

2.1 Pseudo-Random Binary Sequences and Cross-Correlation Method Overview 11

2.2 System Identification of a Plant With Time-Delay 14

2.3 Compensator Tuning for the First-Order Plant With Time-Delay 18

2.4 Compensator Tuning for the Second-Order Plant With Time-Delay 26

2.5 Real-Time Gain Calculation for Systems With Time-Delay 31

3 Validation of the Cross-Correlation and System Identification Portions of the 40 Real-Time Auto Tuning Algorithm

3.1 First-Order PI Compensated Plant With Zero Time-Delay 40

3.2 Second-Order PID Compensated Plant With Zero Time-Delay 45

4 Results of Real-Time Auto Tuning Algorithm on Plants With Time-Delay 50

4.1 First-Order PI Compensated Plant With Normalized Time-Delay $=0.5 \quad 50$

4.2 Second-Order PID Compensated Plant With Normalized Time-Delay $=0.5 \quad 56$

4.3 First-Order PI Compensated Plant With Normalized Time-Delay $=1 \quad 61$

4.4 Second-Order PID Compensated Plant With Normalized Time-Delay = 164

5 Conclusion $\quad 67$ 
6 Discussion $\quad 71$

$\begin{array}{ll}\text { References } & 75\end{array}$

Appendices

Appendix A: Pseudo-Random Binary Sequence Generation for Real-Time Auto Tuning

Appendix B: System Identification Routine Using MATLAB

Appendix C: Algorithm for Real-Time Auto Tuning With Time-Delay

81 


\section{LIST OF FIGURES}

Figure

Page

1 Example System Demonstrating Transport Lag, Modified

from (Ogata, 2002)

$2 \quad$ Algorithm Flow Diagram

3 Block Diagram Showing the System Overview and Its

Application to Time-Delayed Systems

$4 \quad$ Pseudo-Random Binary Sequence Generator and Output

12

Signals Modified from (Isermann \& Münchhof, 2011)

5 Impulse Response of a Second-Order PID Compensated

System With Time-Delay (blue) Compared to the Time

Derivative of the Impulse Response (red)

6 Mean Square Error vs. Pole Parameter for a Second-Order

System With Time-Delay

7 Root Locus for First-Order PI Compensated Plant With

Normalized Time-Delay $=1$ Generated Using Time-Delay

Exponential (Baker, 2011)

8 Root Locus for a First-Order PI Compensated Plant, With

Normalized Time-Delay $=0.5$, Showing Closed-loop Poles

Being Drawn to the Left, Generated Using Time-Delay

Exponential (Baker, 2011)

9 Root Locus Plot for First-Order PI Compensated Plant, With

Normalized Time-Delay $=0.5$, Utilizing the Compensator

Placement Method from Baker's Approach (2011), Generated

Using Time-Delay Exponential

10 Root Locus Plot for a First-Order PI Compensated System

With Normalized Time-Delay $=0.5$, Showing Higher

Performance Generated Using Time-Delay Exponential

(Baker,2011) 
11 Root Locus Plot for Second-Order PID Compensated Plant With Normalized Time-Delay $=0.5$ and Compensator Zero Placement Utilizing Baker Method (2011) Generated Using Time-Delay Exponential

12 Root Locus for a Second-Order PID Compensated System With Normalized Time-Delay $=0.5$ Utilizing the Algorithm Approach Generated Using Time-Delay Exponential (Baker,2011)

13 Root Locus for a Second-Order PID Compensated System With Normalized Time-Delay $=2$ Utilizing the Baker Method (2011) for Normalized Time-Delay $=0.5$ Generated Using Time-Delay Exponential

14 Root Locus for a Second-Order PID Compensated System With Normalized Time-Delay $=2$ Utilizing the Algorithm Method Generated Using Time-Delay Exponential (Baker,2011)

15 Step Response Examples for a First-Order PI Compensated Plant With Normalized Time-Delay Values of $0.5,1$, and 2

16 Step Response Examples for a First-Order PI Compensated Plant With Normalized Time-Delay Values of $0.5,1$, and 2 With a Slower Plant Pole

17 Step Response Examples for a Second-Order PID Compensated Plant With Normalized Time-Delay Values of $0.5,1$, and 2

18 Step Response Examples for a Second-Order PID Compensated Plant With Normalized Time-Delay Values of $0.5,1$, and 2 with a Slower Double Plant Pole

19 First-Order PI Compensated System With Zero Time-Delay

20 System Output for First-Order PI Compensated System With Zero Time-Delay Due to Pseudo-Random Binary Sequence Injection

21 Impulse Response Comparison between MATLAB impulse() Function (blue) and Cross-Correlation Result (red) of a FirstOrder PI Compensated System With Zero Time-Delay 
22 Comparison of Bode Plot for Identified System (red) to Bode

Plot Data from Cross-Correlation (blue) for a First-Order PI

Compensated System With Zero Time-Delay

23 Second-Order PID Compensated System With Zero Time-

Delay

24 System Output for Second-Order PID Compensated System

With Zero Time-Delay Due to Pseudo-Random Binary

Sequence Injection

25 Impulse Response Comparison Between MATLAB impulse()

Function (blue) and Cross-Correlation Result (red) of a

Second-Order PID Compensated System With Zero Time-

Delay

26 Comparison of Bode Plot for Identified System (red) to Bode

48

Plot Data from Cross-Correlation (blue) of a Second-Order

PID Compensated System With Zero Time-Delay

27 First-Order PI Compensated Plant With Time-Delay

28 System Output for First-Order PI Compensated System With

Time-Delay Due to Pseudo-Random Binary Sequence

Injection

29 Estimated Impulse Response of a First-Order PI Compensated

System With Normalized Time-Delay $=0.5$

30 Comparison of Bode Plot for Identified System (red) to Bode

Plot Data from Cross-Correlation (blue) of a First-Order PI

Compensated System With Normalized Time-Delay $=0.5$

31 Step Response Outputs for a First-Order PI Compensated

Plant Tuned for Commanded Overshoots of 0, 5, 10, 15, and $20 \%$

32 Second-Order PID Compensated Plant With Time-Delay

33 System Output for Second-Order PID Compensated System

With Time-Delay Due to Pseudo-Random Binary Sequence Injection 
$34 \quad$ Estimated Impulse Response of a Second-Order PID

35 Comparison of Bode Plot for Identified System (red) to Bode

Plot Data from Cross-Correlation (blue) of a Second-Order

PID Compensated System With Normalized Time-Delay = 0.5

36 Step Response Outputs for a Second-Order PID Compensated Plant Tuned for Commanded Overshoots of 0, 5, 10, 15, and $20 \%$

37 First-Order PI Tuned Step Response for Normalized TimeDelay $=1$ (blue) and De-Tuned Response (green)

38 First-Order PI Compensated System With Oscillatory

Response (blue) and Re-Tuned Response (green)

39 Second-Order PID Tuned Step Response for Normalized

Time-Delay $=1$ (blue) and De-Tuned Response (green)

40 Second-Order PID Compensated System With Oscillatory

66

Response (blue) and Re-Tuned Response (green) 


\section{List of Tables}

Table Page

$1 \quad$ Recommended PI Tuning Relationships for a First-Order 33

Plant With Time-Delay

$2 \quad$ Recommended PID Tuning Relationships for a Second- 34

Order Plant With Time-Delay 


\section{Introduction}

\subsection{A Modern Perspective on Time-Delayed Systems}

Time delay can have a pronounced effect on system operation. For the case of an open-loop dynamic system, many real-world scenarios can influence the control response of the system and cause the system to behave in a way that does not correspond to classical analysis. In many cases, time-delayed systems are idealized and linearized around specific operating regions to combat the time-delay effects (Ogata, 2002). Many examples of these results are summarized by Sipahi, Niculescu, Abdallah, Michiels, and Gu (2011).

Before the advent of modern computing, handling time-delay in control system design required special case design approaches that were very specific to the problem at hand (Ogata, 2002). Pages of hand written calculations and validations were necessary to ensure the design was valid and prototype work would then follow. Design iteration was often required due to an un-modeled parameter that pushed the design out of the intended operating limits. These control problems took many hours to develop mathematical approaches to solve and resulted in designs that were ideal for specific operating conditions. If the conditions were modified, the controller would have to be re-tuned or even re-designed altogether.

Large scale simulation packages have reduced the amount of iterative design required for control problems. Today, any problem can be simulated with an advanced design package. A model can be linear or non-linear, and the simulation software can run with any initial condition, parameter, or model perturbation (Ogata, 2002). Although some of these models are complicated, if there is time to build an accurate model and wait for the simulation iterations to run, a solution that exemplifies what will occur when the system is 
implemented can be attained. Even the waiting times for complex simulation runs are becoming shorter as computational power increases. By analyzing the design in software, and verifying the design for the range of known operating conditions, the designer can then attempt the implementation in hardware with a better chance of proper control behavior (Isermann \& Münchhof, 2011).

The computational approach to design does not only extend to design simulations of products prior to prototype. There is an increasing availability of small, off-the-shelf products that provide users with advanced calculation ability (Miao, Zane, \& Maksimovic, 2004). Computationally intensive non-linear calculations and data analysis can be done on the fly from a device that fits on a small prototype board, something that was unheard of a few short years ago. These products can be leveraged to provide robust control solutions that are highly capable, adaptive, and cheap to maintain.

Time-delayed systems do not follow classical control theory assumptions due to the addition of phase caused by the time-delay exponential (Ogata, 2002). This phase change causes the system to undergo a destabilizing effect as the time-delay increases. The negative feedback process, under the influence of time-delay, inverts the output and feeds it back to the input which causes an amplifying effect. This leads to an oscillatory response and is followed by de-stabilization, which is due to the closed-loop poles moving into the right half plane. This occurs, as was noted by Baker (2011) in his analysis of the time-delay Root Locus, due to the creation of closed-loop poles from the time-delay exponential. As values of the open loop gain increase, the time-delay poles migrate from far in the left half plane to 
areas near the origin of the real axis. These poles repel the dominant closed-loop plant poles into the right half plane, destabilizing the system.

The effect of the time-delay is a linear operator, as noted by Frazzoli (2010).

However, the analysis using more modern compensation approaches is computationally intense. The idea of utilizing modern computing power to compensate for the effects of timedelay needs to be researched and new techniques need to be developed to provide robust compensation for time-delayed systems. 


\subsection{Traditional Methods for Dealing With Time-Delayed Systems}

Time-delay is inherent in many process applications, and virtually all transport process exhibit dead time (Vajta, 2000). The integration of computer systems into compensation solutions also adds a time-delay to the system (Frazzoli, 2010). The effect of this delay can become significant if it is even a small fraction of the system plant time constant, which may be the case in a digital system. Time-delay can also be seen in systems with solid state devices that require complex pulse width modulation. The switching time in these devices can even introduce enough delay to cause instability in the system.

If the time-delay is sufficiently short, it can be ignored for the development of a working controller. Engineers design the controller and are able to tune the controller, via iterative methods, so the time-delay is compensated for properly. One method for iterative tuning is referred to as the Ziegler-Nichols' method (1942). This method is good for a static set of parameters and is sensitive to changes in time-delay. If the time-delay shifts, the system can be driven towards instability.

Another common approach utilized to deal with time-delayed systems is a concept that falls under modern control theory. System estimation is implemented in a specific control application called a Smith Predictor (Bahill, 1983). This control scheme requires good knowledge of the plant model and uses a simulated model that runs in tandem with the

actual plant. By utilizing feedback from the simulated model, the input error is adjusted and the system is controlled as if there was no time-delay. If the time-delay estimate is inaccurate or the time-delay changes, the predictor compensator is subject to the same weaknesses of other static methods. Research has been conducted by Bahill (1983) into automatically 
adjusting a Smith Predictor to utilize an updated time-delay. It is mentioned, however, that this method must obtain an exact representation of the delay for proper operation. This method allows for a larger gain to be maintained but requires a very accurate estimation of the time-delay and system parameters.

Another commonly used approach is the approximation of the time-delay exponential with a polynomial. This approximation is known as the Padé approximation and is based on the Taylor expansion of the time-delay exponential (Vajta, 2000). In theory, by using higher order terms of the Taylor expansion, the Padé approximation provides an estimate of the time-delayed system that will better approximate the dynamics of the real system. This allows for modeling of the system and design of controller parameters using standard root locus methods (Frazzoli, 2010) to compensate for the time-delay. Being an approximation, this approach is also subject to the same issues as the other methods that have been mentioned. As time-delays shift, the approximation will no longer model the system accurately and may even introduce additional error that makes the solution less accurate.

The most common approach to designing around time-delay is to ensure that the system has an adequate phase margin to allow for the time-delay (Ogata, 2002). This approach is based on the Bode Plot analysis of the open-loop system, and the design approach utilizes compensator zeros to boost the phase of the system for higher frequencies. Since the time-delay adds a negative phase to the system, the system is compensated to ensure that the equivalent phase added by time-delay does not exceed the phase margin of the system. 


\subsection{Motivation For Algorithm Development}

This real-time auto tuning algorithm for time-delay systems was inspired by process control problems in industry. The industry standard for process plant compensators relies on the Proportional Integral and Derivative (PID) controller and $95 \%$ of the control loops for process control are controlled via PID (Astrom \& Hagglund, 1995). In many of these systems liquids are heated and transferred from module to module during the process. The fluids vary in temperature, flow, and pressure during the process which leads to complex scenarios that are not always trivial to control. If the system has many operating states, controller tuning will only be valid for a specific state or a range of states. If the system has complex dynamics, including time-delay, the controller will require re-tuning for a change in time-delay to meet the demand of the process.

A simple heater and air transfer system discussed in Ogata's text (2002) is shown in Figure 1. This figure shows a hot air circulation system that transports heated air, through ventilation ducts, to a room. The objective is to control the temperature of the room by heating the air. In this case, the fuel source is governed by the temperature measured by the thermometer. When the room temperature is too low, the furnace applies a heating input to the system. There is a delay in transport of the heated air to the room, which causes a delay in the temperature change in the room. The time-delay will be dependent primarily on the blower speed, because it takes time for the air, traveling at velocity $\mathrm{v}$, to travel the distance L. This leads to a time-delay $\mathrm{T}_{\mathrm{d}}$, given by $\frac{L}{v}$. The temperature output could be controlled with a PID controller or a Smith Predictor (Bahill, 1983), but the system is simple enough to be implemented and tuned for an adequate response without stressing the effects of time-delay. 


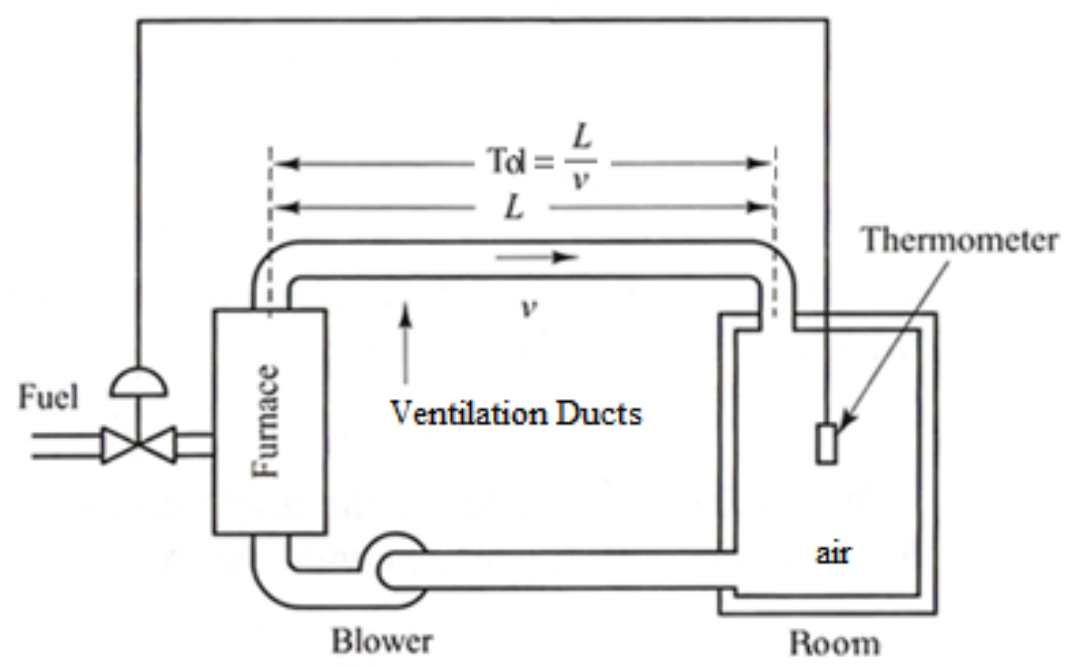

Figure 1 - Example System Demonstrating Transport Lag, Modified from (Ogata, 2002)

This figure provides an example of time-delay. The air takes time to travel from the furnace to the room because of the blower speed. The temperature feedback, for the furnace, is generated from a thermometer in the room. A change in room temperature will cause a change in furnace output. The hotter or cooler air will not reach the room for $T_{d}$ seconds. $L$ refers to the length of the ventilation duct and $v$ refers to the velocity of the air. This delay in the observation of a change in the output variable is known as the transport delay and is primarily dependent on blower speed.

There are numerous variables that can affect how this system operates and is controlled. The temperature sensor could exhibit a time lag or a voltage offset, the furnace could put in more or less heat depending on the temperature of the input air, and there are heat losses from the ventilation ducts during transport of air to the room. These are all examples of things that should be accounted for in the design and operation of the system. Adequate models can be developed and a higher order control scheme can be implemented to account for all of these effects.

One issue that has not been discussed is the flow of the air from the furnace to the room. By an alteration of the air flow speed, $\mathrm{v}$, the system will either be overcompensated or 
undercompensated with respect to the original design specification. For an increase in the time-delay, the furnace heats the air based on the current room temperature for $T_{d}$ seconds with no change in room temperature. This heating is followed by a rapid rise in room temperature, due to the hot air transfer through the ventilation ducts, so the furnace turns off. The room stays overly hot for $\mathrm{T}_{\mathrm{d}}$ seconds, until the room begins to rapidly cool, due to no furnace output. The furnace turns back on to raise the room temperature and the oscillatory cycle repeats. The change in the time-delay has an oscillatory effect on the dynamics of the system, and, if the time-delay is increased with a well compensated system, the system will become unstable. In this case, the result was fluctuation in the output temperature causing probable wear on the furnace and undesirable temperatures in the room. 


\section{Real-Time Auto Tuning Algorithm Development}

This work illustrates a real-time PID auto tuning algorithm for time-delay systems.

The algorithm takes a commanded overshoot from the user and tunes the system to achieve the overshoot. Once the overshoot is selected and delivered to the algorithm, the algorithm will inject a frequency rich signal into the system, cross-correlate the input with the output to achieve an estimate of the impulse response of the system, take the fast fourier transform (FFT) (Oppenheim \& Schafer, 2010) of the estimated impulse response to obtain the estimated frequency response, identify the system transfer function based on known form using a non-linear regression technique, and place the closed-loop plant poles to achieve the desired overshoot in the system step response. This process is shown in Figure 2.

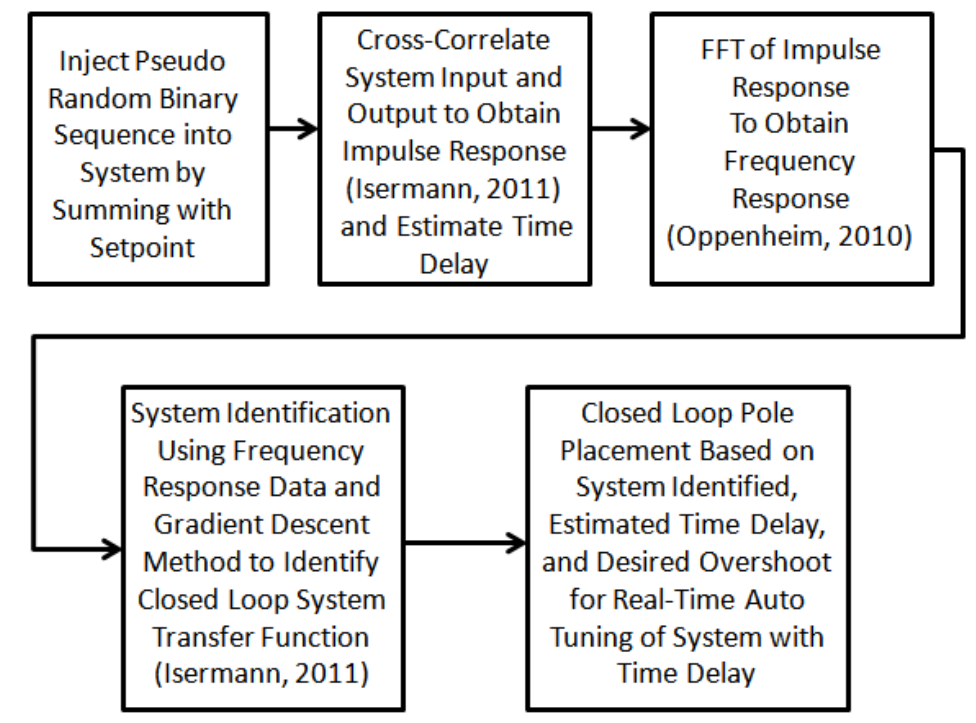

Figure 2 - Algorithm Flow Diagram

This flow diagram shows the steps in the algorithm. Each of these blocks represent a sub portion of the realtime auto tuning algorithm. Although the cross-correlation method for system identification is not new (Miao et al., 2004), this approach has not been applied to the tuning of systems with time-delay. Many of the methods, shown in the blocks, are well studied but the application of placing the closed-loop poles based on the identified system and time-delay has not been adequately addressed. 
This real-time auto tuning algorithm is implemented utilizing the system setup shown in Figure 3. By summing the pseudo-random binary sequence (PRBS) with the system setpoint, the system output is perturbed slightly around the setpoint. The frequency rich PRBS excites the dominant modes of the system and the cross-correlation of the input PRBS and the output of the system provides an estimate of the impulse response (Isermann $\&$ Münchhof, 2011). The FFT of the estimated impulse response is then taken to obtain an estimate of the frequency response of the closed-loop system. The frequency response data is run through a mean square error minimization routine to identify the closed-loop transfer function (Isermann \& Münchhof, 2011). The closed-loop poles are then placed, by adjusting the compensator gain constants in real-time, for the desired overshoot.

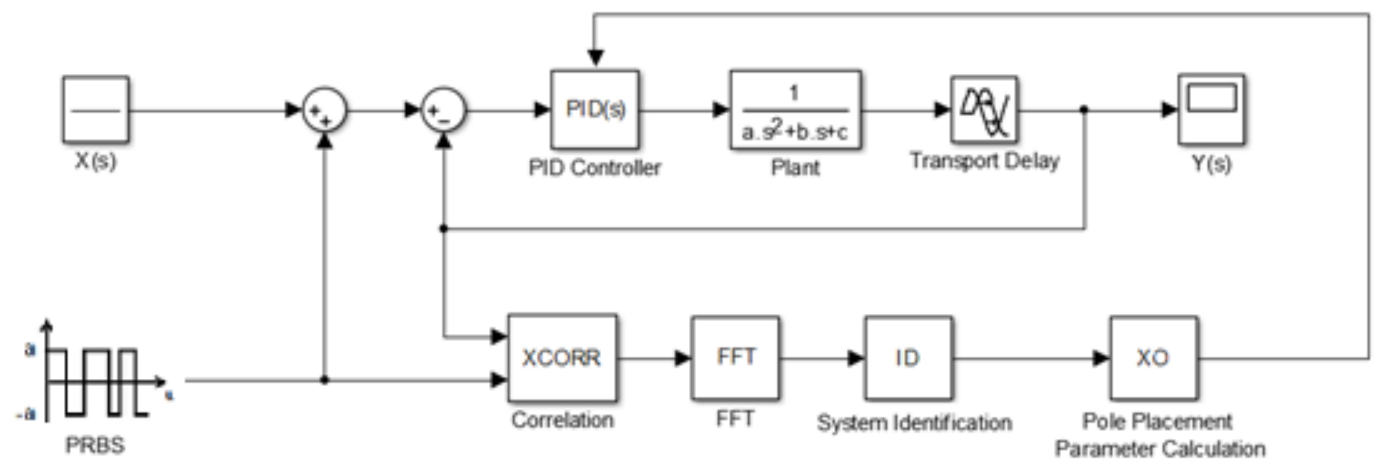

Figure 3 - Block Diagram Showing the System Overview and Its Application to TimeDelayed Systems

This model shows an input setpoint X(s) summed with the PRBS for injection into the closed-loop system. The inputs are then fed forward through the PID compensator, the second-order plant, and the transport delay to produce the output $\mathrm{Y}(\mathrm{s})$. The output $\mathrm{Y}(\mathrm{s})$ is cross-correlated with the input PRBS to produce an estimate of the system impulse response, which is fed through the FFT. This produces an estimate of the frequency response which is used to identify the unknown parameters of the system's transfer function. The compensator zeros are adjusted to compensate the identified system, for a commanded overshoot, with the time-delay accounted for. 


\subsection{Pseudo-Random Binary Sequences and Cross-Correlation Method Overview}

The first step in this real-time auto tuning algorithm involves the use of a frequency rich signal to excite the modes of the system. The ideal choice for this signal is a white noise signal, because it has an infinite frequency bandwidth and is completely random. Upon injection of a white noise signal, all system modes are guaranteed to be excited, which leads to recovery of the impulse response, as shown in the following development (Isermann \& Münchhof, 2011):

$$
R_{x x}(\tau)=S_{x} \delta(\tau)
$$

In Equation (1) $R_{x x}(\tau)$ refers to the auto-correlation function, $S_{x}$ refers to the power spectral density of the white noise signal, and $\delta(\tau)$ refers to the impulse function. The impulse is weighted by the power spectral density of the signal. The cross-correlation function produces the following results:

$$
\begin{gathered}
R_{x y}(\tau)=S_{x} \int_{0}^{\infty} g\left(t^{\prime}\right) \delta\left(\tau-t^{\prime}\right) d t^{\prime}=S_{x} g(\tau) \\
g(\tau)=\frac{1}{S_{x}} R_{x y}(\tau)
\end{gathered}
$$

In Equations (2) and (3), the cross-correlation is denoted as $R_{x y}(\tau)$, the impulse response is denoted as $g(\tau)$, and the dummy variable of integration is defined as $\mathrm{t}^{\prime}$. This result shows that the impulse response is proportional to the cross-correlation of the input white noise signal and the output of the system.

The white noise case, however, is impractical to generate in hardware due to the completely random nature of the signal. To ease the implementation in hardware, a frequency rich signal has been developed which allows for an approximation of the ideal result. This signal is called a PRBS (Isermann \& Münchhof, 2011). The signal is generated by an N-bit 
shift register that utilizes feedback from two of the shift register bits XOR'd together. If the feedback registers are chosen properly, the shift register will cycle through $2^{\mathrm{N}}-1$ states prior to repeating a state. The binary output provided from the shift register is level shifted and scaled to produce a zero mean output. This frequency rich signal exhibits a random nature during a full cycle of the register values and produces a similar result to the ideal white noise case (Isermann \& Münchhof, 2011). An example PRBS generator is shown in Figure 4.

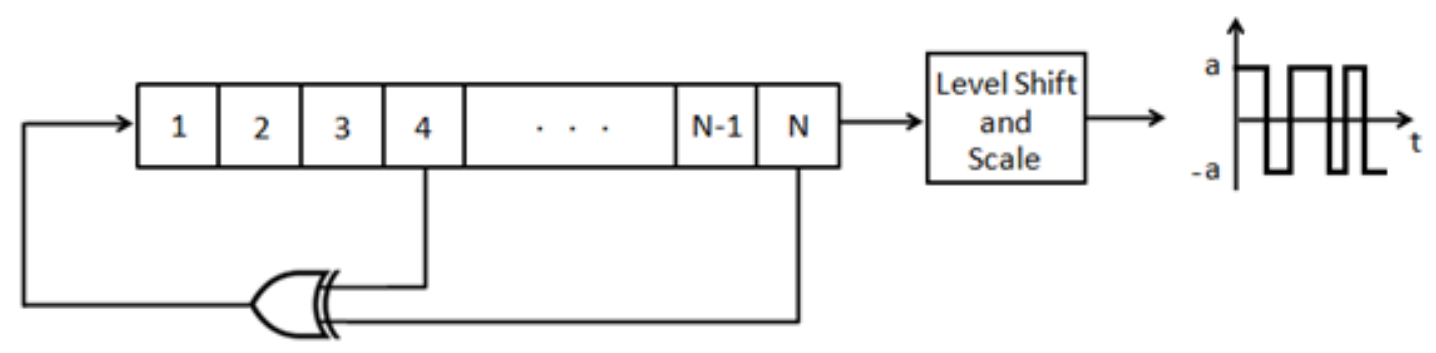

Figure 4 - Pseudo-Random Binary Sequence Generator and Output Signals Modified from (Isermann \& Münchhof, 2011)

This diagram shows the steps in generating a PRBS signal. The shift register is loaded with an initial starting set of bits, a seed. Every clock cycle, the register shifts the bits to the right and the register receives a new bit from the XOR operation. The output is level shifted to take the binary values and create a zero mean signal. This signal is scaled by a value, a, to produce a signal that varies around zero, with an amplitude of a. It is important to note that the output will be a time varying signal that is sufficiently random to be used as a system identification tool (Isermann \& Münchhof, 2011).

The development of this result can be seen in Isermann and Münchhof's work (2011), and the resulting impulse response approximation is defined as follows:

$$
g(\tau)=\frac{1}{a^{2} T_{s}} R_{x y}(\tau)
$$

In this case, $\mathrm{a}$ is the amplitude of the PRBS, $T_{s}$ is the sample time for the signal, and $R_{x y}(\tau)$ is the cross-correlation of the input PRBS and the output of the system. As one can see, this is a similar result with the impulse response being proportional to the cross-correlation. This 
method has been successfully applied to complex systems such as a DC-DC converter in research performed by Miao et al. (2004). A few notes about this result are that the relationship shown in Equation (4) is an approximation of the actual behavior. The resulting equation is based on the assumption that the auto-correlation of the PRBS result is impulselike and the area under the auto-correlation result is treated like an ideal impulse. The smaller the sample time $T_{s}$ with respect to the length of the signal being analyzed, the better the approximation will hold. This is also a periodic signal due to the fact that the PRBS only has $2^{\mathrm{N}}-1$ states. These two facts lead to the conclusion that the analysis time period and the size of the PRBS need to be carefully chosen to provide an adequate estimation of the impulse response.

The PRBS signal is easily implemented in hardware or software and the sequence used in the algorithm uses a 15-bit PRBS generated by a function developed in MATLAB. This function is shown in Appendix A. The function allows for the generation of different sizes of PRBS and provides level shifting, along with specification of the amplitude, of the signal. The reason for choosing 15 bits as the size of the PRBS was to allow for reasonable time and frequency resolution. For the analysis of a 250 second sample of data, this selection allows for evaluation of the system for time-delays of as low as 7.6 milli-seconds and frequency dynamics up to $411.74 \mathrm{rad} / \mathrm{sec}$. This algorithm could be modified to function with systems that have higher frequency dynamics, or smaller time-delays, by changing the length of the sample data or adjusting of the number of bits in the PRBS.

By scaling the cross-correlated output of the system and the input PRBS, the estimated impulse response of the system can be obtained by dividing $R_{x y}(\tau)$ by $a^{2} T_{s}$. The 
estimated impulse response can then be used to find the estimated frequency response of the system. This is accomplished by taking the FFT of the estimated impulse response, and can be utilized for the identification of the system.

\subsection{System Identification of a Plant With Time-Delay}

Once an estimate of the impulse response is obtained from the cross-correlation output, the system can be identified by analysis of the estimated frequency response data. This frequency response data is only an estimate due to the use of a PRBS instead of a white noise signal. However, this data is adequate for proper identification of the system as will be seen in the validation and results sections.

The system identification routine used in this algorithm, is called lsqcurvefit() and is part of the MATLAB optimization toolbox. The routine minimizes the mean square error between a model function and the data provided to the lsqcurvefit() function. In the case of this algorithm, the closed-loop model function, including the second-order double pole plant, PID compensator, and time-delay, is given as:

$$
C L(p, j \omega)=20 \log \left(\left|\frac{G\left(k_{d}(j \omega)^{2}+k_{p}(j \omega)+k_{i}\right)}{e^{(j \omega) T} d(j \omega)((j \omega)+p)^{2}+G\left(k_{d}(j \omega)^{2}+k_{p}(j \omega)+k_{i}\right)}\right|\right)
$$

In this case, $\mathrm{G}$ is the loop gain, $\mathrm{k}_{\mathrm{d}}$ is the derivative gain, $\mathrm{k}_{\mathrm{p}}$ is the proportional gain, $\mathrm{k}_{\mathrm{i}}$ is the integral gain, $\mathrm{p}$ is the double plant pole location, $\mathrm{T}_{\mathrm{d}}$ is the time-delay, and $\mathrm{CL}(p, j \omega)$ is the closed-loop transfer function evaluated at $j \omega$ and $p$. The only parameters that are not known are the time-delay and the plant pole location.

The time-delay of the system can be evaluated utilizing the impulse response estimate of the system, shown in Figure 5. The output of the system will not change due to the PRBS 
input until the time-delay has passed. This result directly translates to the estimated impulse response and allows for the estimation of the system time-delay. The time-delay estimation routine looks at the first peak in the time derivative of the impulse response estimate. The time derivative function will be a maximum at the time the impulse response starts to rise, which is an estimate of the time-delay.

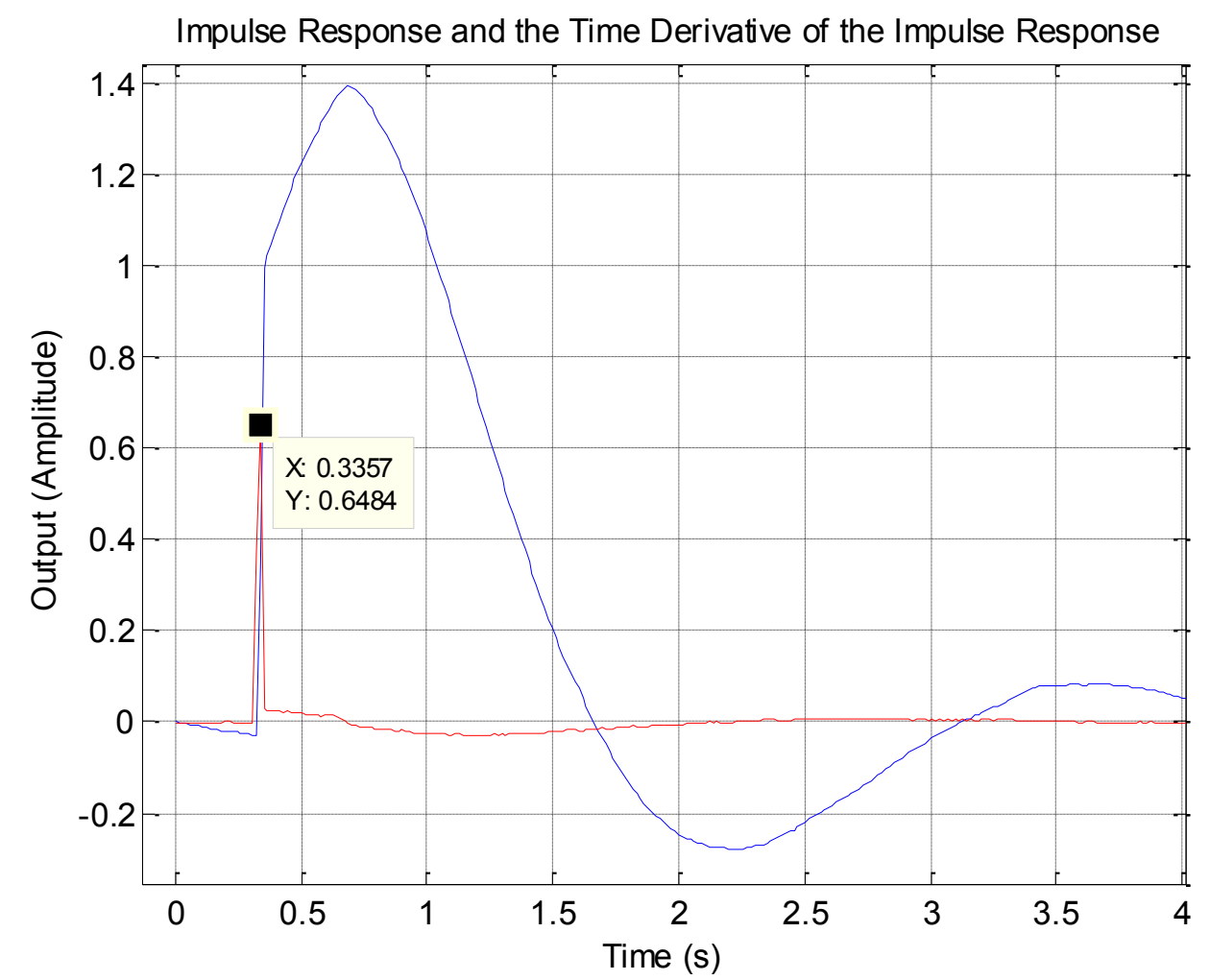

Figure 5 - Impulse Response of a Second-Order PID Compensated System With Time-Delay (blue) Compared to the Time Derivative of the Impulse Response (red)

This figure shows the calculation method for the time-delay estimation. By taking the time derivative of the estimated impulse response, an accurate estimate of the time-delay can be found by looking at the maximum value of the time derivative. This simulation used a second-order plant, with double plant poles at $\mathrm{s}=-3$, and double compensator zeros placed at $s=-4$. The time-delay was 0.33 seconds for this simulation, which is estimated accurately, as 0.3357 seconds. 
This estimation of the time-delay is used in the system identification routine. An application of the time-delay estimation routine is shown in Figure 5 for a second-order, PID compensated, double pole plant with time-delay. The double plant pole is located at $\mathrm{s}=-3$, and the PID compensator zeros are placed at $s=-4$. The time-delay was set to 0.33 seconds and the resulting time-delay estimation found the time-delay to be 0.3357 seconds, which is quite accurate.

The next step in the identification routine is to estimate the system pole location using MATLAB's 1sqcurvefit() function. The function model and the frequency response data are used by lsqcurvefit(), to estimate the unknown pole location parameter, $p$. The Bode plot frequency data is compared to the model function, which yields a mean square error. The difference between the model function and the estimated frequency response data is minimized using the following relationship:

$$
\min \sum_{p}(C L(p)-20 \log (|F F T|))^{2}
$$

In Equation (6), $p$ is the pole parameter, $C L(p)$ refers to the evaluation of equation (6) at $p$, and FFT refers to the data acquired by taking the FFT of the estimated impulse response. It is important to note that the FFT requires $2^{\mathrm{N}}$ samples and the data obtained, during one PRBS cycle, only contains $2^{\mathrm{N}}-1$ sample points. This leads to a situation where the FFT will be padded by one zero in the calculation, which is reasonable in comparison to the large set of data being utilized.

This process requires a starting guess at the parameter to allow convergence towards the lowest mean square error. The process for the regression method involves making an initial guess for which way to increment the pole parameter, evaluating whether the step was 
in a direction that reduces the mean square error, and deciding which way to step next to continue to cause a decrease in mean square error. This is referred to as the gradient descent method and is discussed in Isermann and Münchhof's text (2011). This process is repeated until the change in mean square error between iterations reaches a specific tolerance value or the number of function evaluations exceeds a pre-set limit. This is to ensure the function times out in a situation where the result does not converge.

If the initial guess for the system uses a large pole parameter value, there is still a chance that the routine will not find the actual pole location. There is also a local minimum other than the global minimum for the error. This local minimum occurs at a parameter value of zero as can be seen in Figure 6.

Figure 6 exhibits the behavior of the mean square error for a large range of pole parameters. In this case, the system was of second-order with time-delay and the double plant poles were located at $\mathrm{s}=-15$, which is seen as the global mean square error minimum. If the algorithm took too large of a step as it approached the global minimum, it may have found the local minimum at $\mathrm{s}=0$ instead. This problem is alleviated by the algorithm adjusting step size based on the change in mean square error between iterations. This ensures that the initial steps are large and the steps near the solution become smaller to converge quickly to a solution and prevent missing of the global minimum. See Appendix B for the MATLAB code used in the identification routine. 


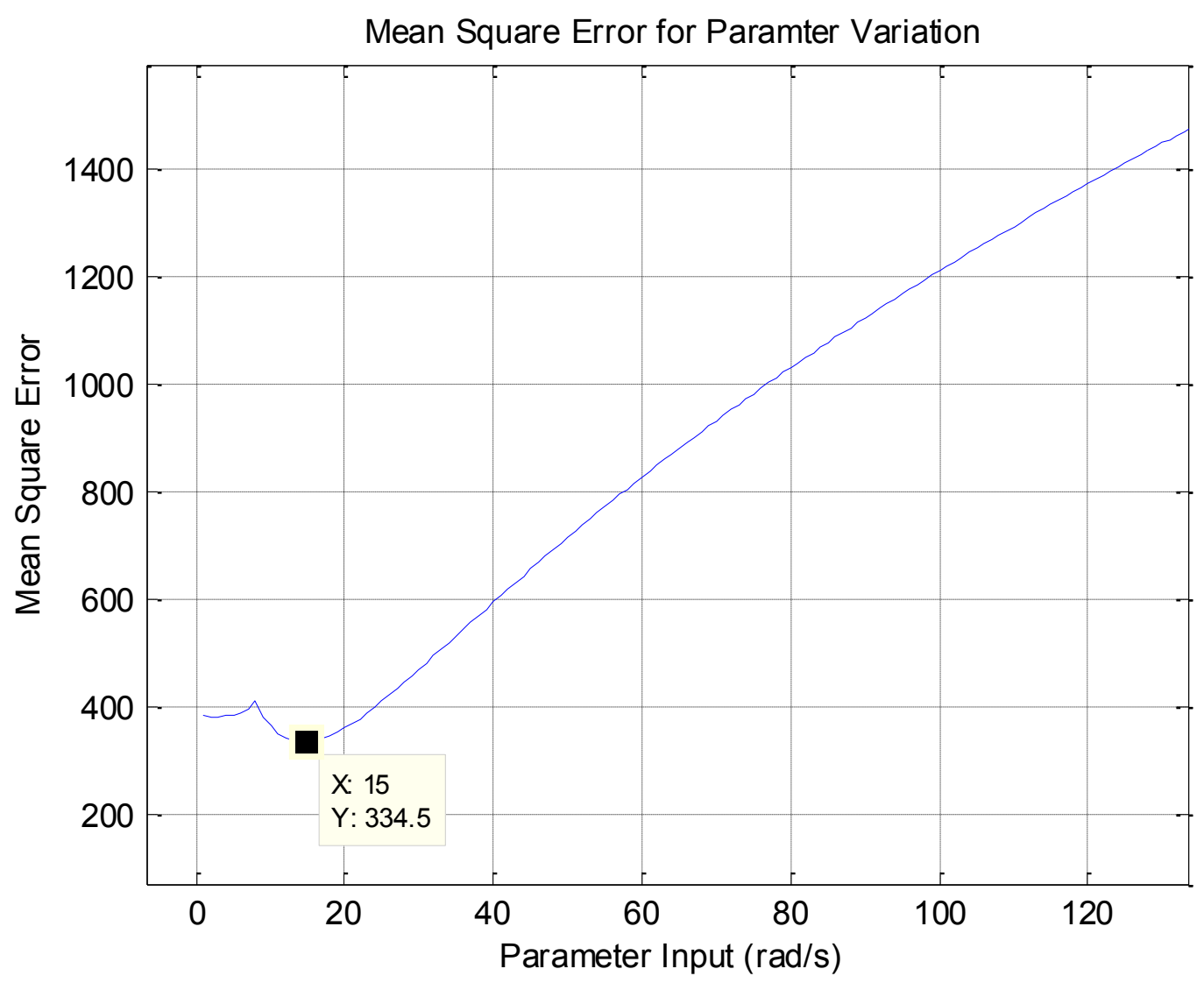

Figure 6 - Mean Square Error vs. Pole Parameter for a Second-Order System With TimeDelay

Example mean square error curve for identifying a second-order system with system poles at $s=-15$. This curve shows that the mean square error continues to rise as the estimated plant pole is evaluated at higher frequencies. The graph also shows that there is a global minimum at the identified pole where $\mathrm{s}=-15$ and a local minimum at $s=0$. A large initial guess for the system pole is a good starting point since the gradient descent technique will likely find the global minimum.

\subsection{Compensator Tuning for the First-Order Plant With Time-Delay}

Once a transfer function model has been determined, the closed-loop poles must be placed to achieve the desired overshoot. This concept was explored by Baker (2011) for time-delay values that fall into a reasonable class. In this class, the closed-loop poles can be moved far enough into the left hand plane to produce an accelerated system response. For 
values outside this range, the time-delay effects on the system adversely affect the Root Locus, causing an inability to move the closed-loop poles far enough to the left to achieve a faster system response.

The development of this method requires the defining of a ratio known as normalized time-delay (NTD) which is the ratio of the time-delay inherent in the system, $\mathrm{T}_{\mathrm{d}}$, versus the time constant of the open loop plant pole T (Astrom \& Hagglund, 1995). This relationship is shown in the following equation:

$$
N T D=\frac{T_{d}}{T}
$$

The range of NTD that Baker's method (2011) analyzed included NTD of 0.05 to 0.5. The real-time auto tuning algorithm accommodates the same range of NTD, as discussed in Baker's method, but extends the idea to larger values of NTD. In these cases, the closed-loop poles will not be able to be placed far enough into the left half plane to accelerate the system response, as shown in Figure 7 for an NTD of 1. However, the system will still be tunable to achieve a desired overshoot, by sacrificing the response time.

Due to the nature of the time-delayed system, traditional Root Locus methods cannot be applied unless time-delay poles are accounted for. These pole loci run roughly parallel to the real axis, and occur with spacing of $\frac{2 \pi}{T_{d}}$ radians due to the effects of the complex exponential. For NTD values greater than 0.5 , the pole loci begin to come close enough to the plant poles to have a drastic effect on the Root Loci, as shown in Figure 7. In many cases, the compensator zeros that are added into the system will no longer attract the plant poles. The compensator zeros will now attract the poles created by the time-delay. This leaves the plant poles to be driven into the right half plane causing instability for even moderate gain 
values. This behavior is shown for an example first-order system, with time-delay of 0.25 seconds and NTD $=1$. In this case, the closed-loop plant poles migrate to instability at a gain of approximately 5.2. In this case, the PI compensator added will not accomplish the goal of a faster response for the system since there is no way to move the closed-loop plant poles further into the left half plane.

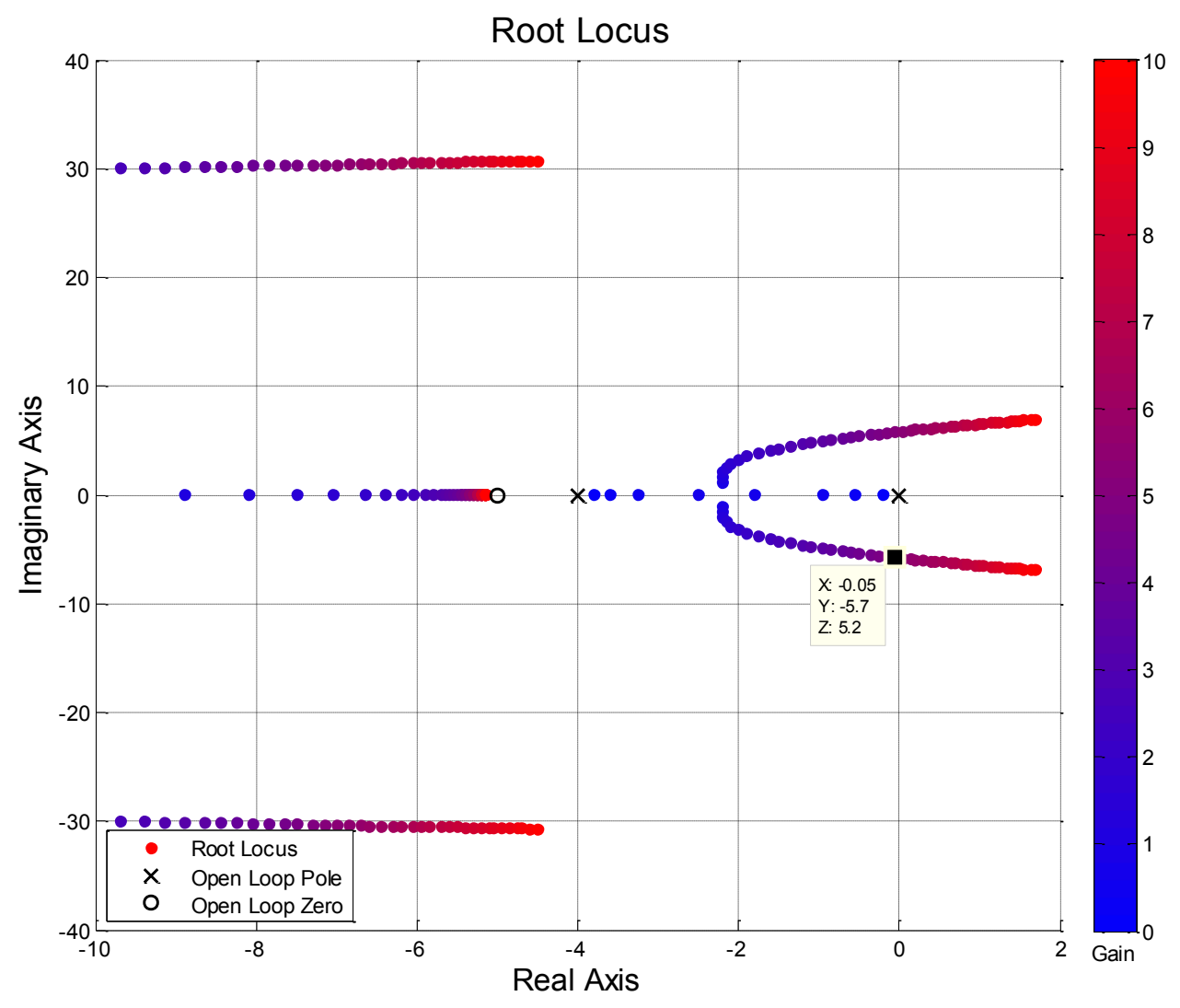

Figure 7 - Root Locus for First-Order PI Compensated Plant With
Normalized Time-Delay $=1$
Generated Using Time-Delay Exponential (Baker, 2011)

Root Locus plot showing behavior of a first-order plant with a plant pole at $\mathrm{s}=-4$ and a compensator zero placed at $\mathrm{s}=-5$. This figure shows that the closed-loop plant poles are no longer able to be drawn to the left by the compensator zero. Instead, they migrate into the right half plane and exhibit marginal stability at a gain of 5.2. The figure also shows the time-delay poles separated by $\frac{2 \pi}{T_{d}}=25.12$. The time-delay poles have been repelled by the plant poles to a value of approximately 30 . 
In the case of NTD $<0.5$, the effects from the time-delay poles still do occur but the compensator zero still has the ability to attract the closed-loop poles for moderate gain values (Baker, 2011). As the gain gets higher, the time-delay poles migrate from far in the negative left half plane to the right and this causes the closed-loop plant poles to migrate into the right half plane. With the plant pole at $\mathrm{s}=-4$ and with $\mathrm{NTD}=0.5$, the compensator is able to draw the closed-loop plant poles to the left of the open loop plant poles, as shown in Figure 8. As gain is raised, the time-delay poles begin to show their effect and the closed-loop plant poles migrate towards instability. 


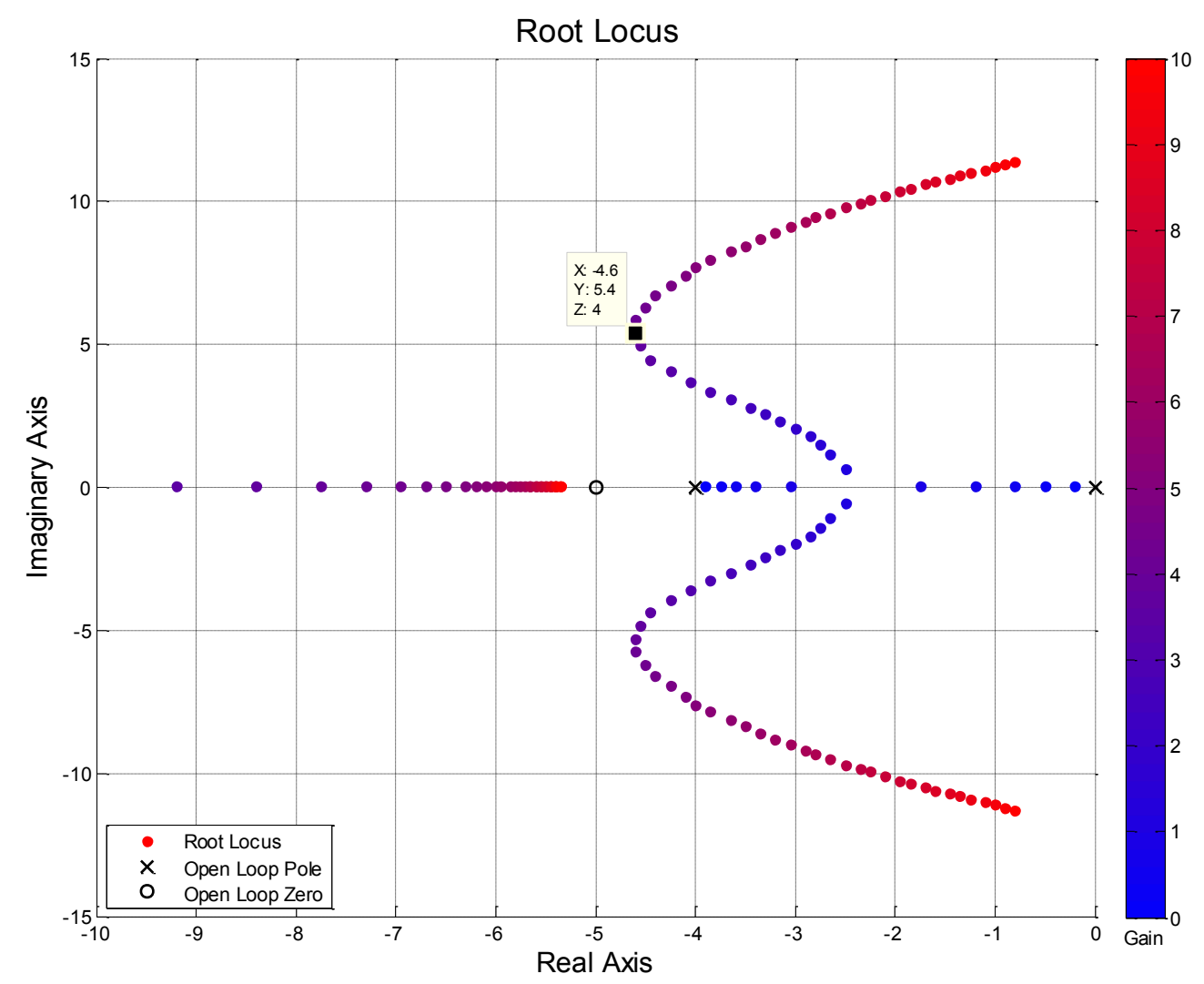

Figure 8 - Root Locus for a First-Order PI Compensated Plant, With

Normalized Time-Delay $=0.5$, Showing Closed-loop Poles Being Drawn to the Left, Generated Using Time-Delay Exponential (Baker, 2011)

Root Locus plot showing the closed-loop poles being drawn further into the left half plane, which will make the system response quicker. This will also cause the system to ring due to the complex conjugate poles. Baker's method (2011) utilized this approach to draw the poles further into the left half plane and then reduced the gain to balance the overshoot and load disturbance rejection. The real-time auto tuning algorithm focuses on targeting a commanded overshoot. The plant pole for this system is located at $s=-4$ and the compensator zero is located at $\mathrm{s}=-5$. The saddle point of the loci occurs at a gain of 4 and this gain places the dominant closed-loop poles at $\mathrm{s}=-4.6 \pm 5.4 \mathrm{j}$.

Utilizing Baker's approach (2011) to tune the system for quick response and overshoot reduction, a PI compensator zero is placed at 1.5 times the plant pole for an NTD of 0.5 . This places the zero at $s=-6$ with a recommended loop gain of 1 to achieve the response shown in Figure 9. 


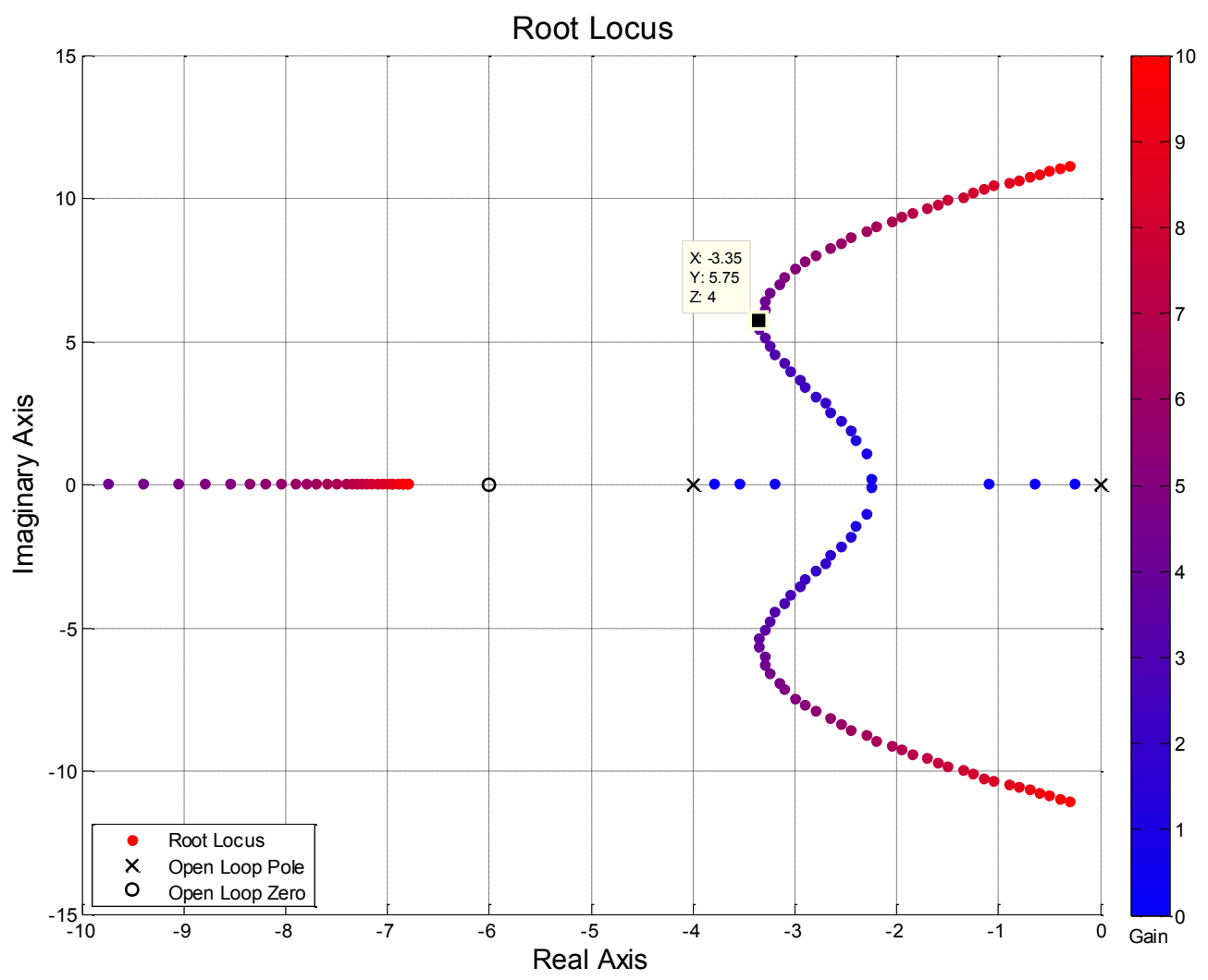

Figure 9 - Root Locus Plot for First-Order PI Compensated Plant, With Normalized Time-Delay $=0.5$, Utilizing the Compensator Placement Method from Baker's Approach (2011), Generated Using Time-Delay Exponential

Root locus demonstrating the compensator zero placement using Baker's method (2011). This method involves the placement of the zero at 1.5 times the plant pole which is located at $s=-4$, for an NTD of 0.5 . This places the compensator zero at $s=-6$. In this case, the closed-loop plant poles are not drawn very far into the left half plane which leads to a result that is not optimum for time response performance. In this compensator placement, the saddle point places the closed-loop poles at $\mathrm{s}=-3.35 \pm 5.75 \mathrm{j}$ which does not provide a performance increase over the open loop plant poles. This system has an inherent time-delay of 0.125 seconds.

An important note for this Root Locus is that the closed-loop poles for this plant are stable with relatively low overshoot and no steady state error. However, even by properly adjusting the gain to the saddle of the loci, as shown in the figure for a loop gain of 4 , the closed-loop poles of the system will not provide a faster response than the open loop poles of 
the system. At a gain of 4, the closed-loop poles are drawn as far as possible into the left half plane and reach a value of $\mathrm{s}=-3.35 \pm 5.75 \mathrm{j}$. If the goal of adding a PI compensator is merely system stability and setpoint tracking, this would be an adequate solution. However, in many cases, performance is one of the most important factors in the design.

In order to adjust the system closed-loop poles to achieve a desired overshoot and ensure an adequate performance gain with no steady state error, the compensator PI zero must be moved closer to the open loop plant pole. This allows for a greater attraction between the pole and zero and allows for a larger draw of the closed-loop poles into the left half plane, before being affected by the time-delay poles. To keep a reasonable separation of the compensator zero and pole, the compensator zero will be placed at 1.1 times the plant pole. This consistent pole placement for various NTD values will allow more opportunity for the Root Loci to be drawn to the left, allowing for a faster system response. The Root Locus that exemplifies this pole placement scenario is shown in Figure 10, which shows a large performance gain over the Baker method (2011). Because of this performance gain, this is the method of compensator zero placement that will be applied in the real-time auto tuning algorithm. This system has a plant pole at $\mathrm{s}=-4$ and a compensator zero placed at $\mathrm{s}=-4.4$, with a time-delay of 0.125 seconds. 


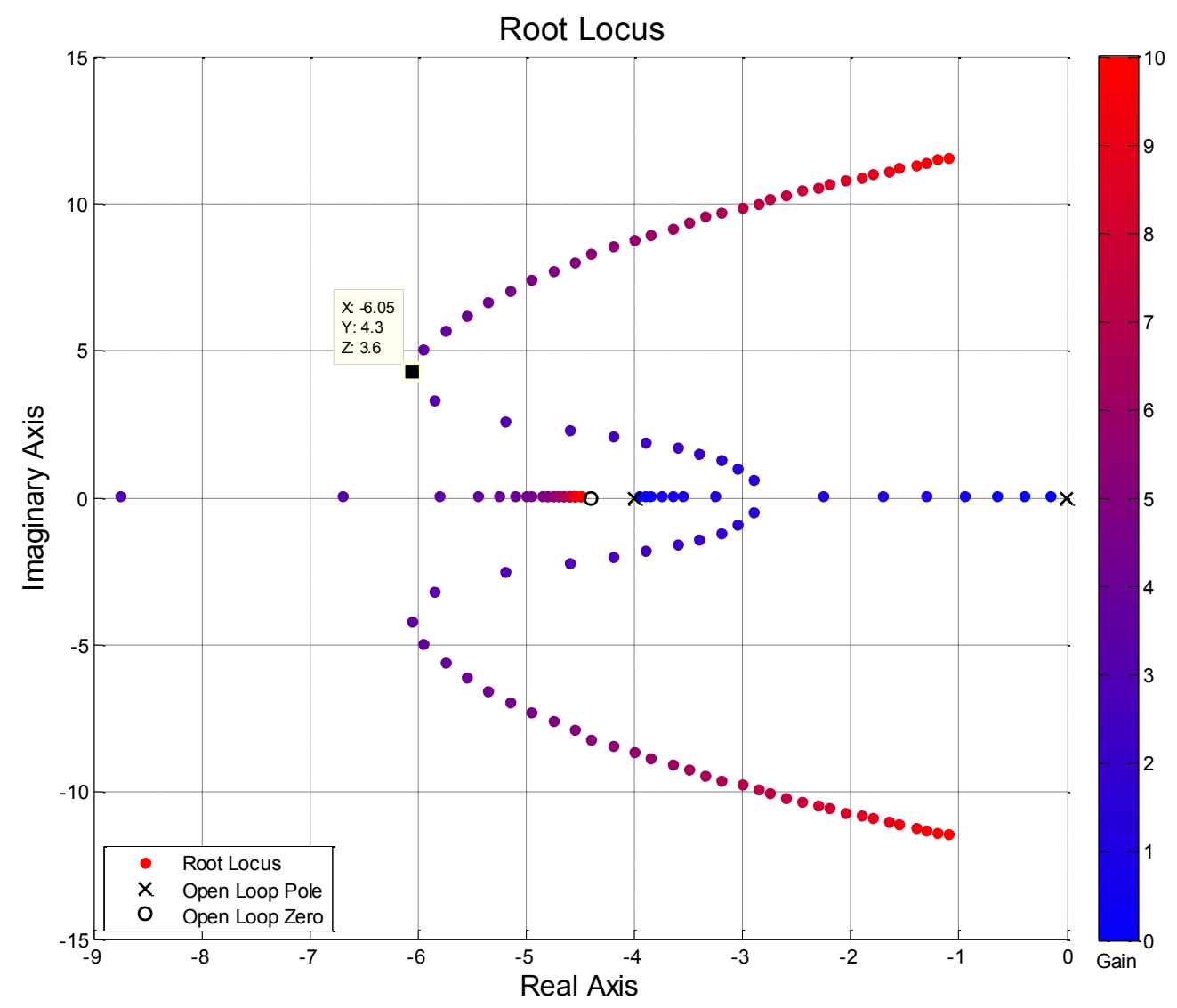

Figure 10 - Root Locus Plot for a First-Order PI Compensated System With Normalized Time-Delay $=0.5$, Showing Higher Performance

Generated Using Time-Delay Exponential (Baker,2011)

This figure shows the modified pole placement method that demonstrates the capability of providing higher performance in a first-order PI compensated system. The plant pole is located at $\mathrm{s}=-4$ and, by placing the pole at 1.1 times the location of the open loop pole, the Root Locus is drawn further to the left. This compensator zero placement shows a marked performance increase over the results shown in Figure 9, with the closed-loop plant poles being drawn to $\mathrm{s}=-6.05 \pm 4.3 \mathrm{j}$ at a gain of 3.6. The point selected on the Loci in the figure shows the fastest system response achievable with this system for an NTD of 0.5 . 


\subsection{Compensator Tuning for the Second-Order Plant With Time-Delay}

This research focused on the compensation of a double pole plant. In a second-order PID compensated plant, the closed-loop poles are moved further into the left half plane by the compensator double zero placement. This case is similar to the first-order case but the maximum acceleration of the plant response will also be dictated by the closed-loop pole that travels from the double pole position towards the compensator zero along the real axis. One of the closed-loop plant poles migrates in each direction along the real axis. The pole migrating to the left, approaches one of the compensator zeros and the other moves to the right, where it collides with the integrator pole. The two closed-loop poles then break away from the real axis. The closed-loop poles are forced into the right half plane for higher gains due to influence from the time-delay poles. There is also a time-delay pole that migrates from negative infinity towards the second compensator zero for higher gains.

Just as in the single pole plant case, there will be an attempt to apply the Baker method (2011) to accomplish adequate tuning of the system, by moving the closed-loop plant poles as far to the left as possible. The Baker method recommends placing the double compensator zeros at 1.06 times the double pole position. This will be at $\mathrm{s}=-3.18$. This corresponds to PID compensator values of $\mathrm{k}_{\mathrm{d}}=1, \mathrm{k}_{\mathrm{p}}=6.36$, and $\mathrm{k}_{\mathrm{i}}=10.1124$. In this case, the Baker method recommends a gain of 1.7. The optimum acceleration of the plant response would occur at the saddle point of the graph which occurs at a loop gain of 2.8 , as shown in Figure 11. 


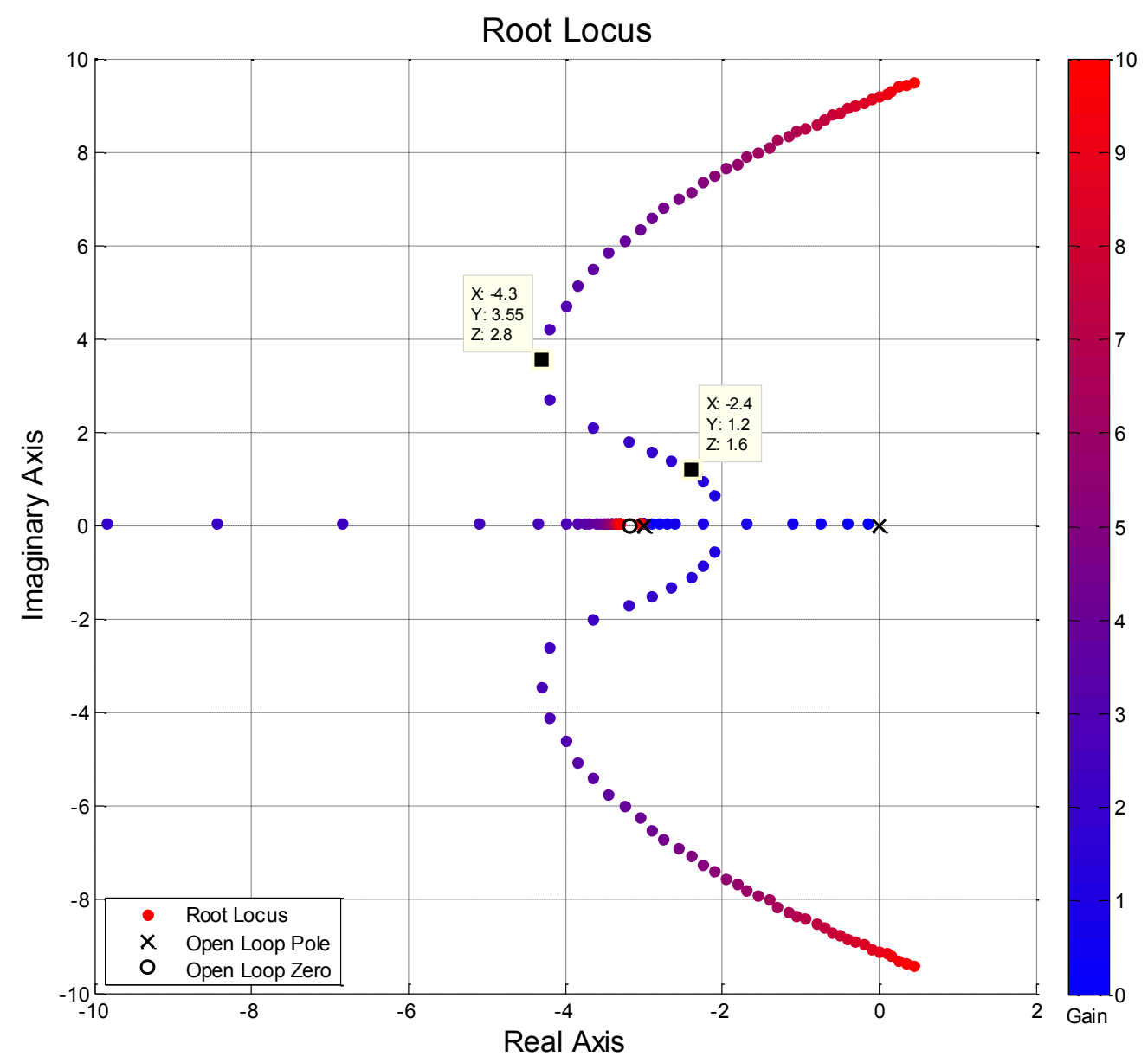

Figure 11 - Root Locus Plot for Second-Order PID Compensated Plant With Normalized Time-Delay $=0.5$ and Compensator Zero Placement Utilizing Baker Method (2011) Generated Using Time-Delay Exponential

Root Locus plot showing the application of Baker's method (2011) for a second-order PID compensated plant with NTD of 0.5 . In this case, the optimum plant response acceleration occurs at a gain of 2.8 , while Baker's method recommends a gain of 1.7, for overshoot reduction and disturbance rejection. The plant involves a double pole at $s=-3$ and a double compensator zero placed at $s=-3.18$. The time-delay for this system is 0.167 seconds.

In the case of the second-order plant, the Baker method (2011) provides an ample double compensator zero placement to move the closed-loop poles further into the left half plane. However, the gain adjustment needs to be modified to allow for a balance between 
system response and overshoot. To apply a consistent method, as in the single pole plant case, it is important to keep the ratio of the double plant pole location to the PID compensator zeros constant. This allows for predictable behavior over a larger range of NTD values. After experimenting with compensator zero placement locations, it was noticed that the compensator zeros can more aggressively compensate the system when placed on either side of the double plant pole for large values of NTD. The compensator zero locations were chosen as 1.1 and 0.9 times the double plant pole location. This compensator placement leads to a scenario where the integrator pole is attracted to the compensator zero, to the right of the double plant poles, the time-delay zero comes from negative infinity and is attracted towards the zero to the left of the plant poles, and the two plant poles break away immediately from the real axis to be driven to the right by the time-delay poles. This allows for a best case closed-loop performance that occurs at the open loop zero location to the right of the plant poles, as shown in Figure 12. This does not create much of a performance increase over the Baker approach (2011) for ranges of NTD between 0.05 and 0.5 , but allows for better tuning when the time-delay is on the order of the plant time constant.

For large NTD, this compensator zero placement method provides a notable performance increase over the Baker method (2011). Take for example the same plant with an NTD of 2. Utilizing the pole placement method applied by Baker yields the best case closed-loop performance at a double plant pole of $\mathrm{s}=-1.15$. This is $38 \%$ of the performance of the open loop poles. By applying the pole placement method used in the algorithm, the best case plant pole placement for performance occurs at $\mathrm{s}=-1.85$. This is $61.7 \%$ of the open loop plant time constant so this method will be utilized in the development of the auto tuning 
algorithm. This Root Locus behavior is shown in Figure 13 for the Baker method (2011) and Figure 14 for the method used in the algorithm. It is important to note that the Baker method was not developed for NTD greater than 0.5 and the result is only included for comparison.

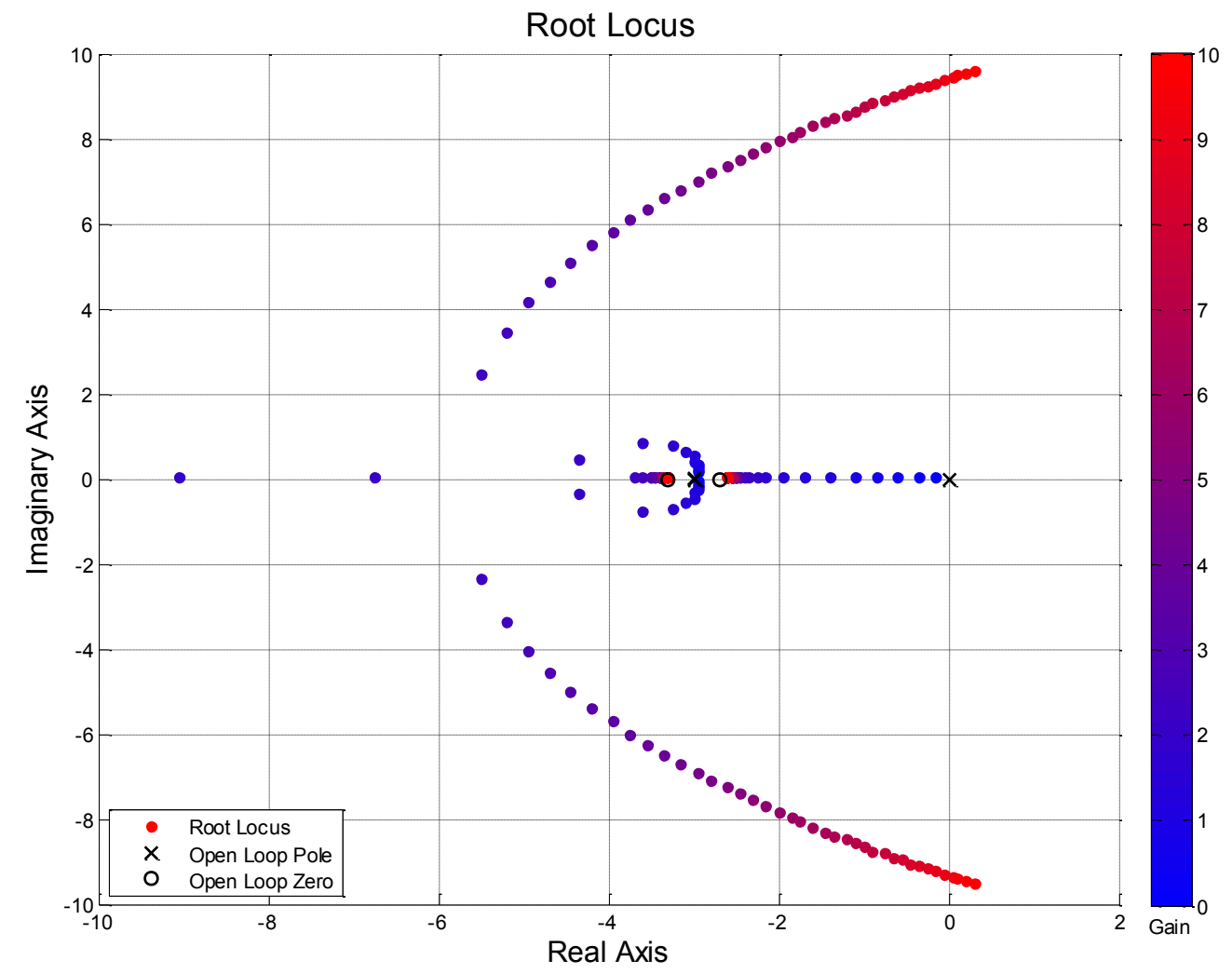

Figure 12 -Root Locus for a Second-Order PID Compensated System With Normalized Time-Delay $=0.5$ Utilizing the Algorithm Approach

Generated Using Time-Delay Exponential (Baker,2011)

This Root Locus plot shows that the compensator zero placement, at 0.9 and 1.1 times the plant poles, applied in the algorithm does not show performance gains over Baker's method (2011) for NTD on the order of 0.5. The figure does show that there is a much larger draw of the closed-loop poles into the left half plane which is leveraged for NTD values greater than 0.5 . In the case of this plant, with compensator zeros placed at $\mathrm{s}=-3.3$, -2.7 , the response of the system is still limited by the migration of the integrator pole. 


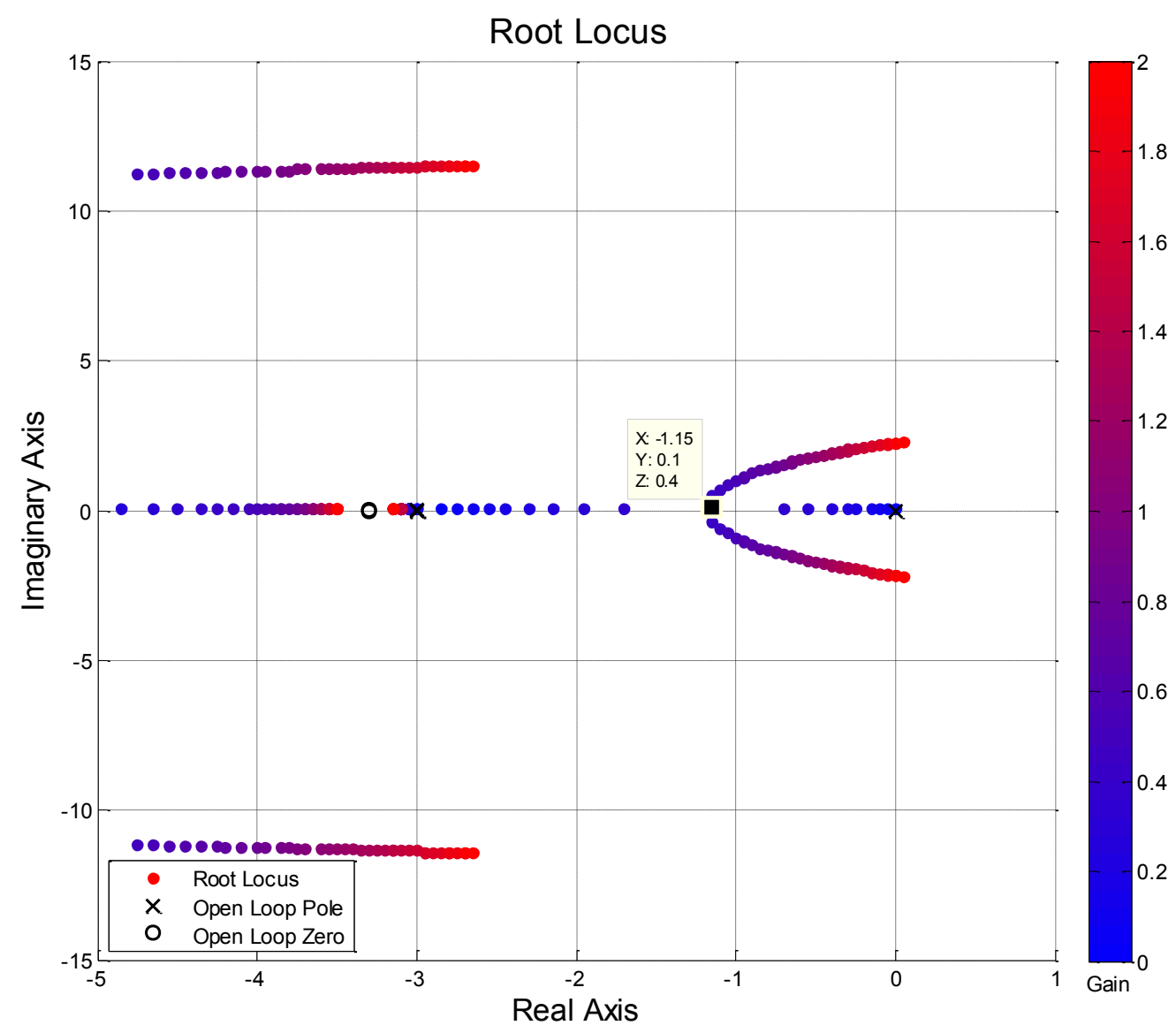

Figure 13 - Root Locus for a Second-Order PID Compensated System With Normalized Time-Delay $=2$ Utilizing the Baker Method (2011) for Normalized Time-Delay $=0.5$ Generated Using Time-Delay Exponential

Root Locus plot showing the performance of Baker's compensator zero placement method (2011) for the double pole system with $\mathrm{s}=-3$. In this case the compensator double zero is placed at $\mathrm{s}=-3.18$ which is 1.06 times the double plant pole. The fastest system response is achievable at the break away point where the closed-loop plant poles are located at $\mathrm{s}=-1.15$, which is $38 \%$ of the open loop plant pole time constant. It is important to note that Baker's method does not cover NTD in this region, but the result is shown for comparison. 


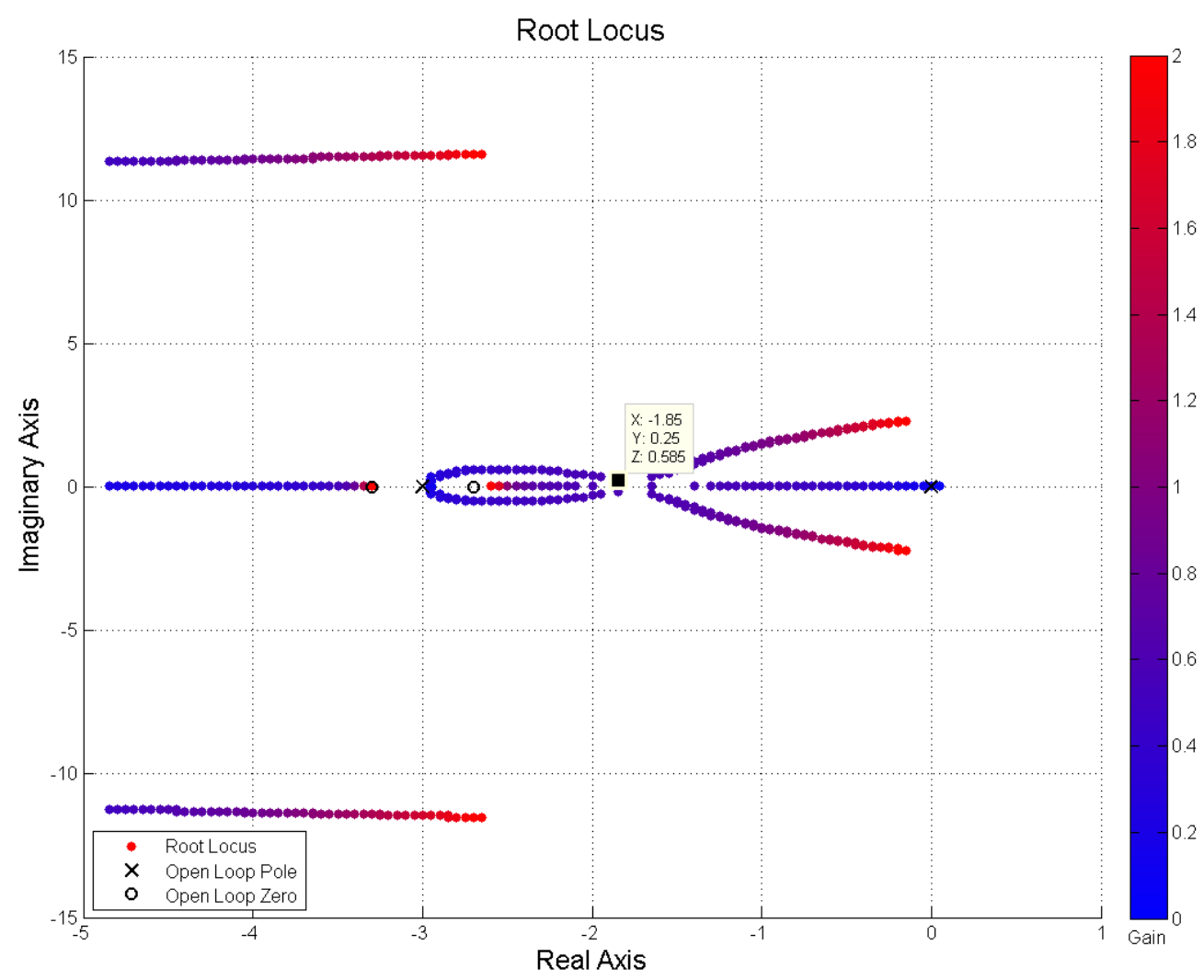

\section{Figure 14 - Root Locus for a Second-Order PID Compensated System With Normalized Time-Delay $=2$ Utilizing the Algorithm Method \\ Generated Using Time-Delay Exponential (Baker,2011)}

This Root Locus shows the performance gain using the new compensator placement technique. The plant is still a double pole plant with $\mathrm{s}=-3$, but the compensator zeros are placed on either side of the plant poles at 1.1 and 0.9 times the double pole location. This causes the plant poles to immediately break away from the real axis which causes higher performance to be achieved, before the closed-loop poles migrate to the right. This is a large improvement over Baker's method (2011), which produced closed-loop plant poles at $\mathrm{s}=-1.15$. This method produced closed-loop plant poles at $\mathrm{s}=-1.85 \pm 0.25 \mathrm{j}$.

\subsection{Real-Time Gain Calculation for Systems With Time-Delay}

The next portion of the compensator adjustment relies on the automatic determination of gain to achieve the proper overshoot. This portion of the algorithm relies on a close estimate of the plant pole location to ensure that the compensator zero is placed at the 
appropriate location for the Root Locus to be predictable. If there is error in the identified pole location, or in the estimation of the system time-delay, the gains calculated by this method will not be as accurate as desired.

To develop the set of gain relationships summarized in Table 1 and 2, a first-order system and a second-order system with time-delay were iteratively tuned to achieve the desired overshoot response for various values of time-delay. The loop gain for each of these tests was recorded and plotted against the value of NTD to determine a relationship between the gain and the NTD of the system for each overshoot. The relationship between gain and NTD ended up being an inverse relationship in which gain could be calculated based on a constant and the inverse of NTD. This result was further reduced and can be calculated using the time-delay and the overshoot commanded. The developed parameters used in the algorithm are based on linear interpolations of the region between two overshoot values. The MATLAB program that calculates the overshoot is shown in Appendix C. Once the system is identified and the time-delay has been estimated, the gain that needs to be applied and the adjustment of the compensator zeros comes from calculations in Tables 1 and 2. These gain calculations have been included in the real-time auto tuning algorithm so there is no work that needs to be done by the user, other than supplying a commanded overshoot. The algorithm assesses all of the system parameters and applies the appropriate gain to the system based on the assessed time-delay and overshoot desired. 


\section{Table 1}

Recommended PI Tuning Relationships for a First-Order Plant With Time-Delay

\begin{tabular}{cc}
\hline Overshoot $(\%)$ & Gain \\
\hline $0-4$ & $\frac{0.02 * \text { Overshoot } \%+0.35}{T_{d}}$ \\
$4-10$ & $\frac{0.015 * \text { Overshoot } \%+0.36}{T_{d}}$ \\
$10-20$ & $\frac{0.0115 * \text { Overshoot } \%+0.39}{T_{d}}$ \\
$20-30$ & $\frac{0.01 * \text { Overshoot } \%+0.41}{T_{d}}$ \\
Greater than 30 & $\frac{0.0097 * \text { Overshoot } \%+0.425}{T_{d}}$ \\
\hline
\end{tabular}

This table provides recommended gain calculations for a first-order PI compensated system, based on overshoot and time-delay inherent in the system, utilizing a compensator zero placement at 1.1 times the plant pole. 


\section{Table 2}

Recommended PID Tuning Relationships for a Second-Order Plant With Time-Delay

\begin{tabular}{cc}
\hline Overshoot (\%) & Gain \\
\hline $0-4$ & $\frac{0.02 * \text { Overshoot } \%+0.42}{T_{d}}$ \\
$4-10$ & $\frac{0.0133 * \text { Overshoot } \%+0.4467}{T_{d}}$ \\
$10-20$ & $\frac{0.0115 * \text { Overshoot } \%+0.47}{T_{d}}$ \\
$20-30$ & $\frac{0.011 * \text { Overshoot } \%+0.475}{T_{d}}$ \\
Greater than 30 & $\frac{0.01 * \text { Overshoot } \%+0.5}{T_{d}}$ \\
\hline
\end{tabular}

This table shows recommended gain calculations, utilizing a commanded overshoot and a system time-delay, for a second-order PID compensated plant with zero placements at 1.1 times the double plant pole and 0.9 times the double plant pole location.

It is desirable to be able to choose a tradeoff between overshoot and time response, so the algorithm was developed to accommodate an automatic choice of gain based on the desired overshoot. The idea behind this approach is that a user can specify the overshoot that the system can support and the best response gain will be chosen for the current plant, the compensated PID, and the current system time-delay. To illustrate the performance of the method for a first-order PI compensated system, Figures 14 and 15 show the step responses for various selected overshoots in specific NTD situations. As one can see from the figures, the results are fairly consistent over a range of NTD. By improving this relationship and 
finding a function to describe the relationship between overshoot, NTD, and tuned gain, the responses could be idealized to match perfectly in all scenarios. As the algorithm stands today, the responses are not ideal due to linear interpolations utilized in the development of the algorithm. One important aspect of this approach is that the computation requirement is much lower than an analytical solution and is feasible with simple mathematical operations.

One important aspect of this approach is that there is no accelerating of the system response for larger values of NTD. However, the plant response matches the expected value of overshoot while maximizing the performance, for this compensator placement method. In the case of large NTD, the time-delay poles push the system towards instability. With a traditional PI or PID compensator, the best that can be accomplished is to stabilize the system and make a compromise for overshoot if time performance is required.

Utilizing the iterative tuning approach, a set of equations was also developed for a second-order PID compensated system. In this case, the poles are placed on either side of the plant poles offset by $\pm 10 \%$ of the open loop plant pole value. Assuming this pole placement, a gain calculation is performed which will yield the appropriate overshoot for the given NTD. An example of the step responses for various requested overshoots and NTD values is shown in Figures 17 and 18, for a second-order plant. Note that this set of responses was much easier to perform linear interpolation for the data due to the symmetric nature of the zero placement. It does not exhibit the same issues as the method applied to the first-order plant. 


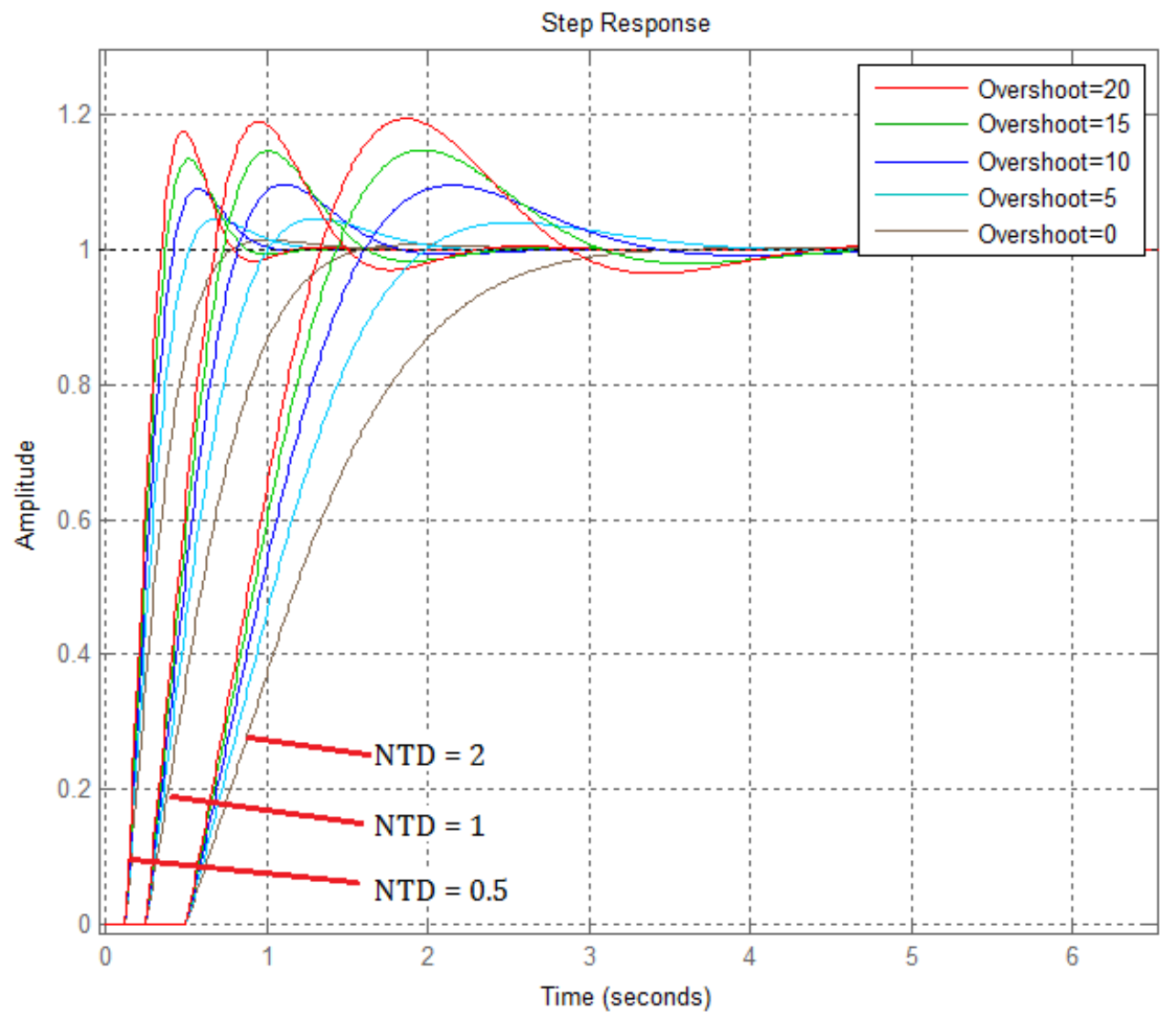

Figure 15 - Step Response Examples for a First-Order PI Compensated Plant With Normalized Time-Delay Values of 0.5, 1, and 2

This figure shows the response of a test system with a plant pole at $\mathrm{s}=-4$ and a compensator zero placed at $\mathrm{s}=$ -5 . In this case, the commanded overshoot is met within 5\% for NTD values between 0.5 and 2 . This exemplifies the fact that there is a relationship between the pole placement and choice of loop gain for the system. The method shows a slight difference between the commanded overshoot and the actual overshoot for low values of NTD on the order of 0.5 but the results are consistent for higher NTD values. 


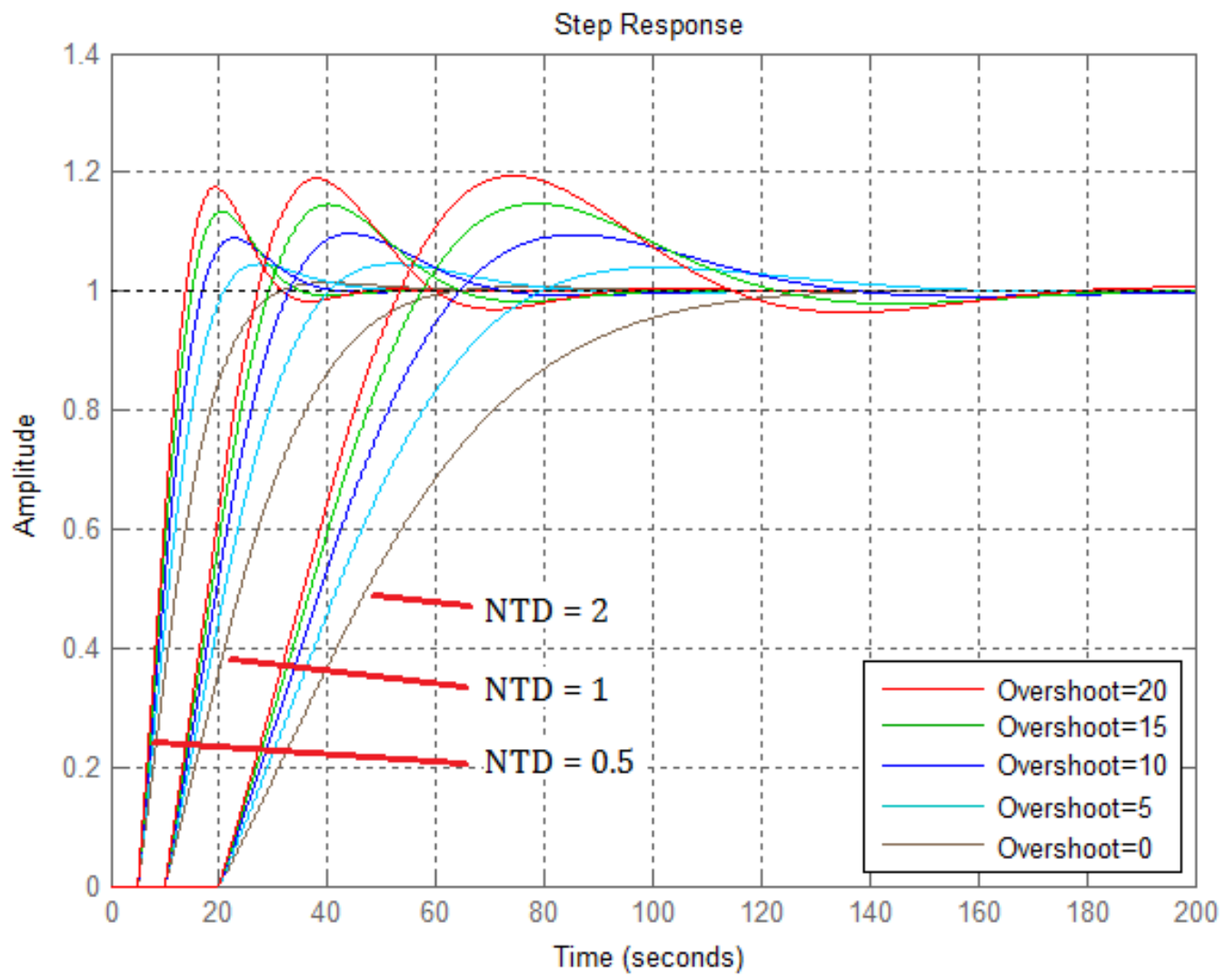

Figure 16 - Step Response Examples for a First-Order PI Compensated Plant With Normalized Time-Delay Values of 0.5, 1, and 2 With a Slower Plant Pole

This figure shows that the method is independent of the pole location. By maintaining the compensator zero placement at 1.1 times the plant pole, $s=-0.11$, the results are comparable to those found in Figure 15, with a plant pole located at $\mathrm{s}=-0.1$. In this case, the system exhibits a slower response due to the plant pole but is capable of achieving the commanded overshoot. This result shows that the algorithm is independent of the plant pole location. 


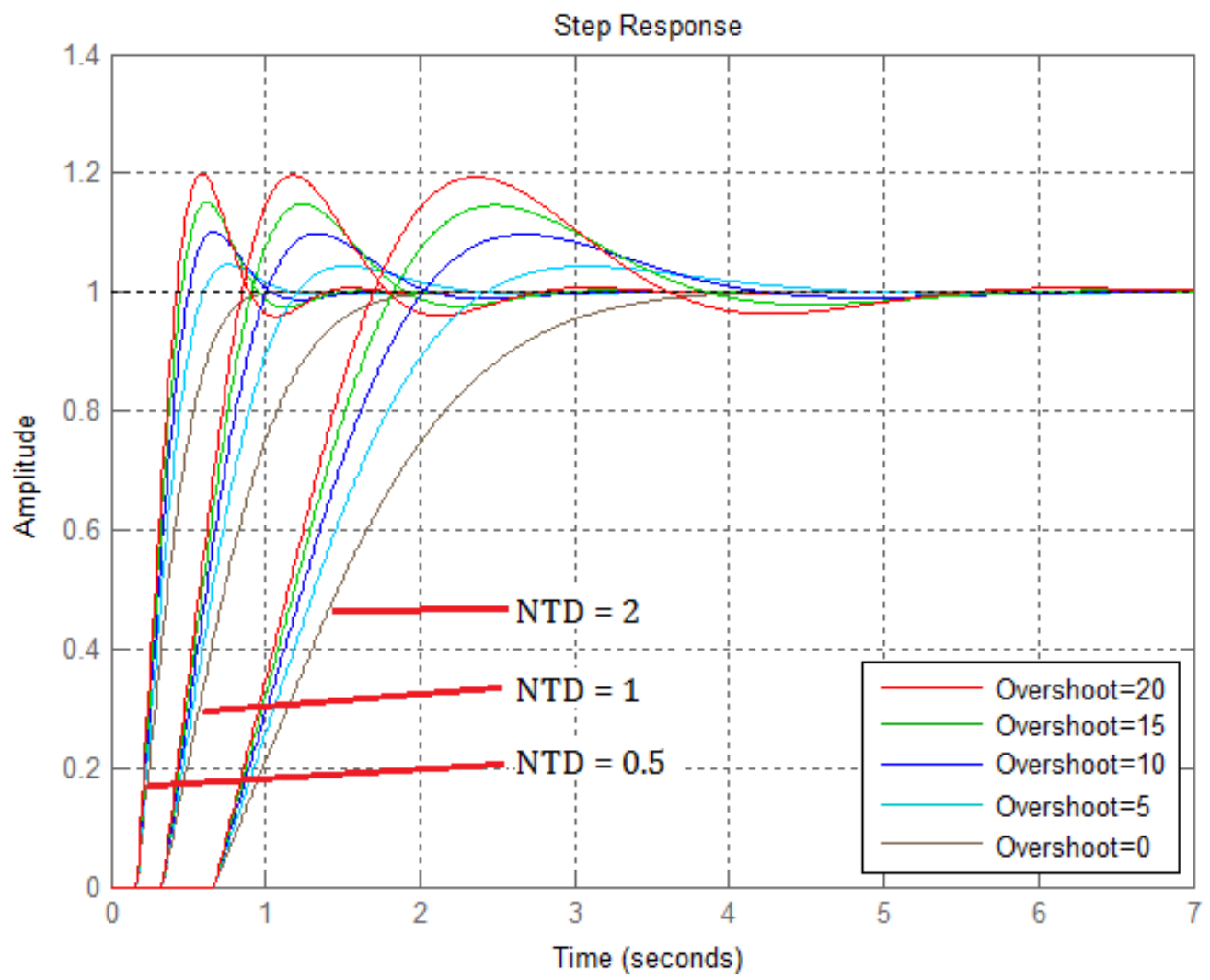

Figure 17 - Step Response Examples for a Second-Order PID Compensated Plant With Normalized Time-Delay Values of 0.5, 1, and 2

This figure illustrates that compensator zero placements of 1.1 and 0.9 times the open loop plant pole location, $\mathrm{s}=-3$, yield satisfactory overshoot responses for NTD from 0.5 to 2 . In this case, commanded overshoot matches the ideal overshoot perfectly. 


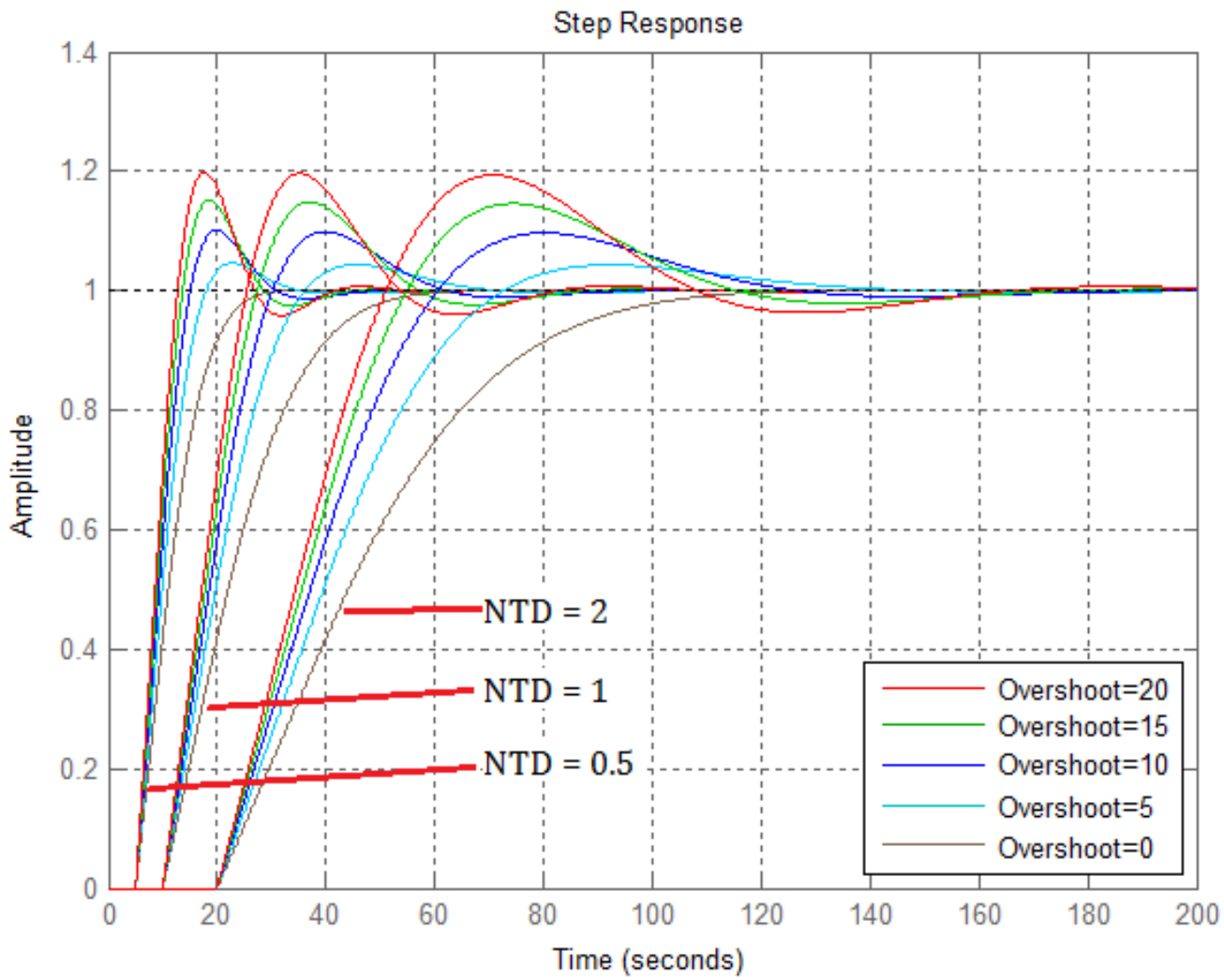

Figure 18 - Step Response Examples for a Second-Order PID Compensated Plant With Normalized Time-Delay Values of 0.5, 1, and 2 With a Slower Double Plant Pole

This figure is used to exemplify that the method is independent of pole location and shows comparable results with respect to the last example. In this case, the NTD is varied between 0.5 and 2 and the double plant pole has been relocated to $\mathrm{s}=-0.1$. The system response is much slower due to the double plant pole location. 


\section{Validation of the Cross-Correlation and System Identification Portions of the Real- Time Auto Tuning Algorithm}

This chapter is devoted to the validation of the auto-tuning algorithm by comparing the output of the algorithm to known classical results. The algorithm involves steps that can be generalized to plants that do not exhibit time-delay, including the injection of the PRBS and the identification of the system's closed-loop plant poles. The algorithm is validated on a first-order PI compensated plant and a second-order PID compensated plant, with time-delay set to zero.

\subsection{First-Order PI Compensated Plant With Zero Time-Delay}

The first-order PI compensated plant is shown in Figure 19. The remainder of the algorithm blocks including PRBS injection, cross correlation of input and output to estimate the impulse response, and the identification of the system have not been included in this figure but are shown in Figure 3.

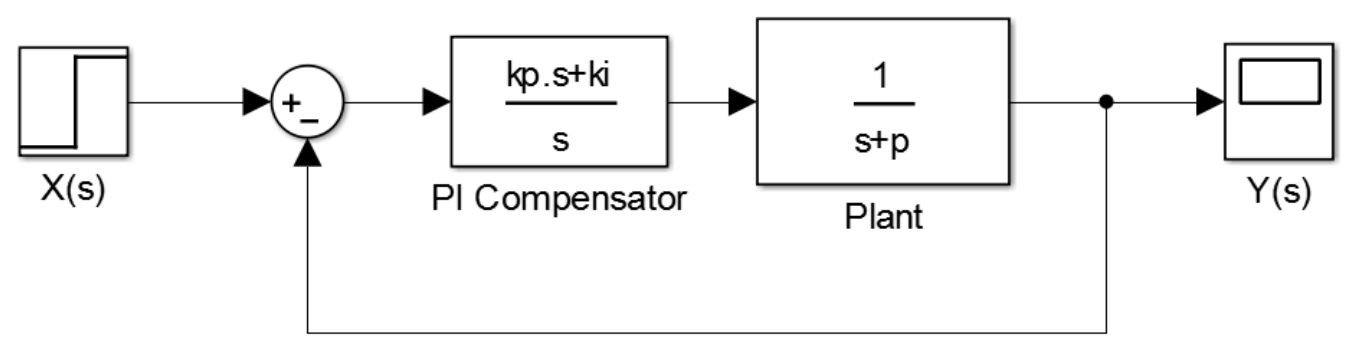

Figure 19 - First-Order PI Compensated System With Zero Time-Delay

This figure shows the first-order plant utilized for the validation of the algorithm. This plant does not have timedelay so the algorithm is applied with time-delay set to zero. The plant is a single pole plant with plant pole at $\mathrm{p}$, proportional gain $\mathrm{k}_{\mathrm{p}}$, and integral gain $\mathrm{k}_{\mathrm{i}}$. The algorithm portions of the system are not shown but include PRBS injection, cross correlation of input and output, and system identification, as shown in Figure 3. 
The closed-loop transfer function, including PI compensator and a single plant pole, is given as:

$$
C L(s)=\frac{k_{p}\left(s+\frac{k_{i}}{k_{p}}\right)}{s^{2}+\left(k_{p}+p\right) s+k_{i}}
$$

In this case, $\mathrm{k}_{\mathrm{p}}$ is the proportional gain, $\mathrm{k}_{\mathrm{i}}$ is the integral gain, and $\mathrm{p}$ is the plant pole location. The closed-loop transfer function is noted as CL(s).

The test system utilized for the first-order PI compensated system with time-delay set to zero, has a plant pole at $\mathrm{s}=-5$, a compensator zero at $\mathrm{s}=-4$, and a loop gain of 1 . A 15-bit PRBS with amplitude of 1 was injected into the system over a time period of 250 seconds to identify the system. One-hundred seconds of the output of the system due to PRBS injection are shown in Figure 20. The estimated impulse response is compared to the impulse response computed by the MATLAB impulse() function in Figure 21. 


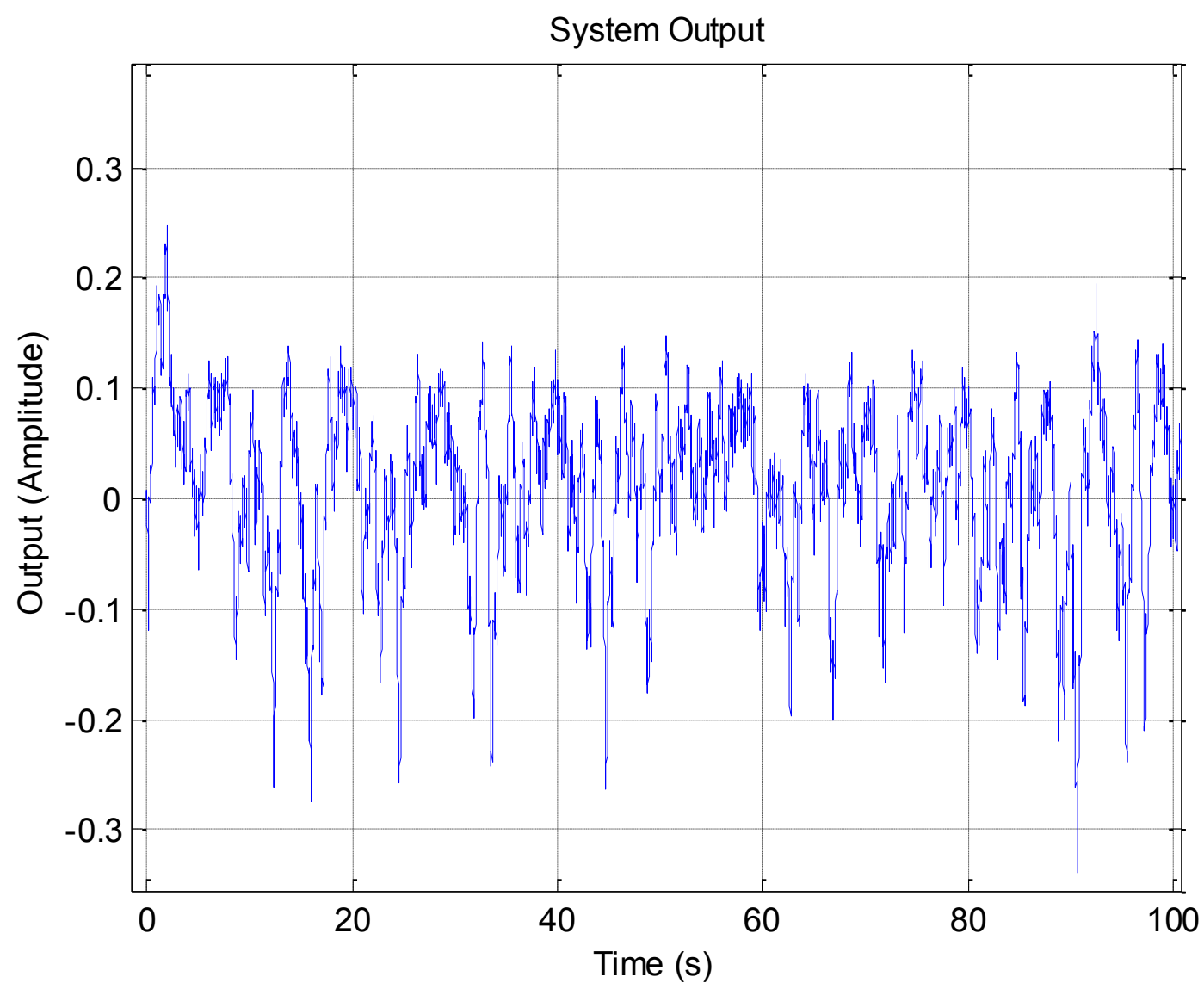

Figure 20 - System Output for First-Order PI Compensated System With Zero Time-Delay Due to Pseudo-Random Binary Sequence Injection

This figure shows the system output when a 15-bit PRBS with an amplitude of 1 is injected into the test system. This system has a loop gain of 1 , a compensator zero placed at $\mathrm{s}=-5$, a plant pole at $\mathrm{s}=-4$, and time-delay set to zero. 


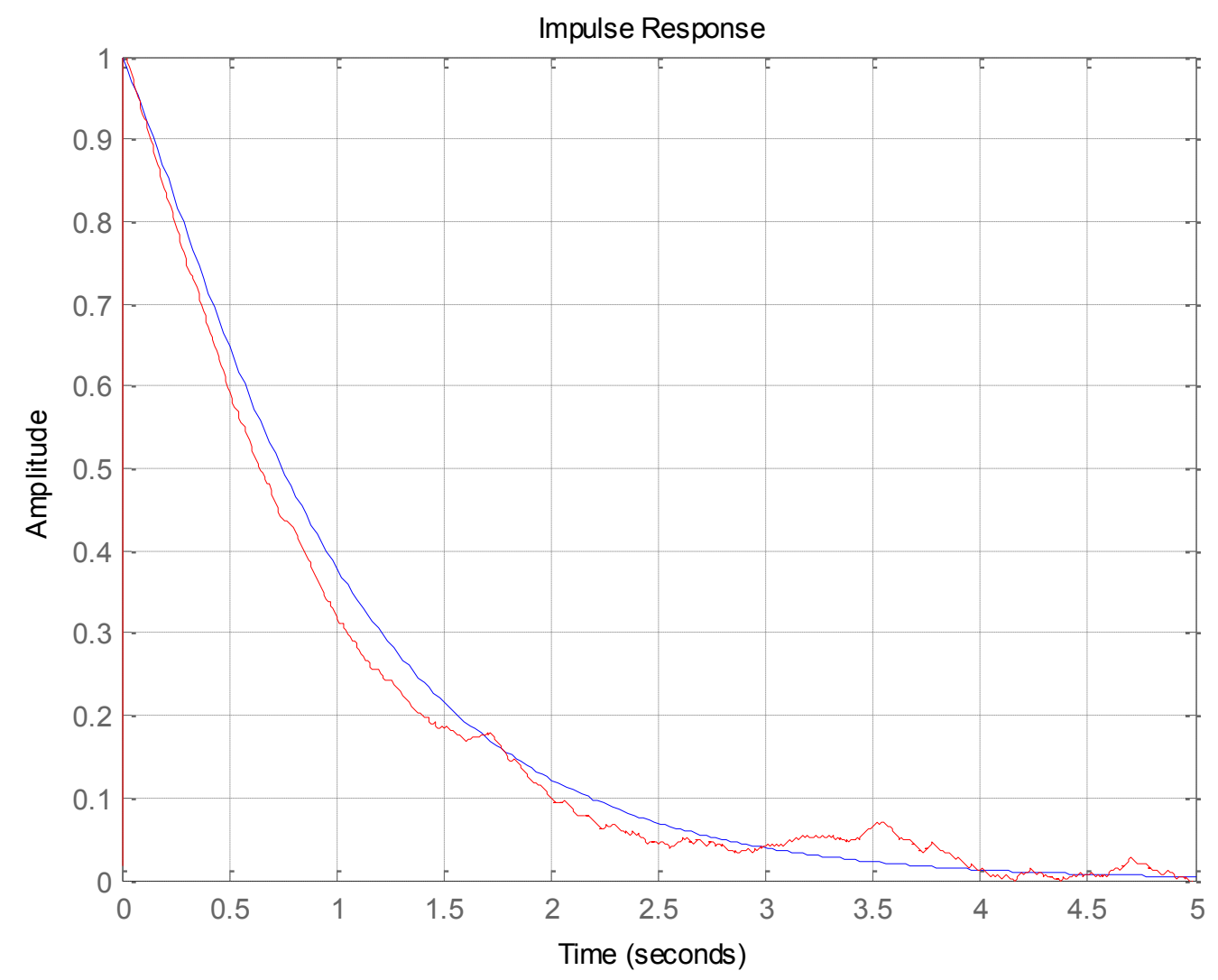

Figure 21 - Impulse Response Comparison between MATLAB impulse() Function (blue) and Cross-Correlation Result (red) of a First-Order PI Compensated System With Zero TimeDelay

This figure shows the estimated impulse response from the cross correlation output in red, compared to the impulse response from the MATLAB impulse() function in blue. This system has a loop gain of 1, a compensator zero placed at $\mathrm{s}=-5$, a plant pole at $\mathrm{s}=-4$, and time-delay set to zero.

The system identification routine identified the open loop plant pole at $\mathrm{s}=-3.8082$ with the actual plant pole being located at $\mathrm{s}=-4$. This validates the use of PRBS injection, cross correlation of input and output to obtain the impulse response estimate, and the proper identification of the system using the MATLAB lsqcurevefit() function, for a first-order 
system with time-delay set to zero. The Bode plot of the frequency response is shown in

Figure 22.
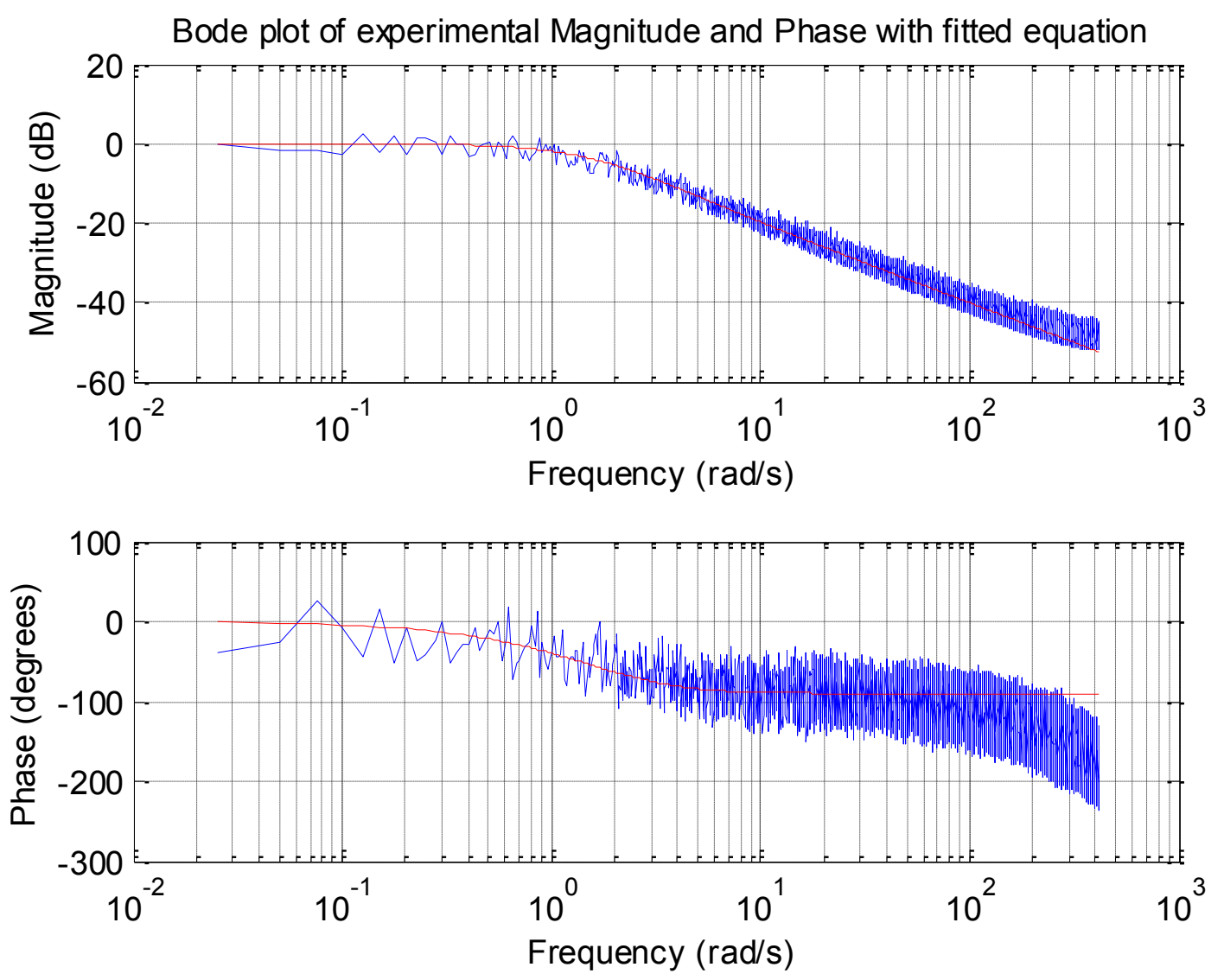

Figure 22 - Comparison of Bode Plot for Identified System (red) to Bode Plot Data from Cross-Correlation (blue) for a First-Order PI Compensated System With Zero Time-Delay

This figure shows that the identified system pole at $\mathrm{s}=-3.8082$ matches the actual system pole at $\mathrm{s}=-4$. The identified system Bode plot is shown in red compared to the estimated frequency response data which is shown in blue. The close match is shown by the fact that the identified system response runs through the center of the estimated frequency response data. 


\subsection{Second-Order PID Compensated Plant With Zero Time-Delay}

The second-order PID compensated plant is shown in Figure 23. The remainder of the algorithm blocks including PRBS injection, cross correlation of input and output to obtain the impulse response estimate, and the system identification blocks have not been included in this figure but are shown in Figure 3. The plant is given in the general form but the system tested in this analysis involves a second-order plant with a double pole.

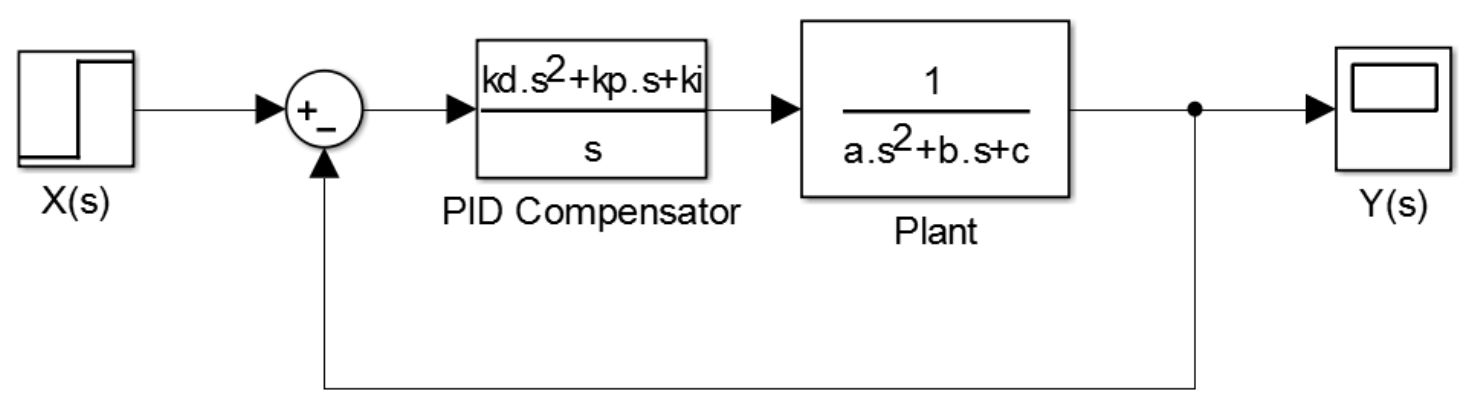

Figure 23-Second-Order PID Compensated System With Zero Time-Delay

This figure shows the second-order plant utilized for the validation of the algorithm. This plant does not have time-delay so the algorithm is applied with time-delay set to zero. The plant is a double pole plant with plant poles at $\mathrm{p}$, a derivative gain $\mathrm{k}_{\mathrm{d}}$, proportional gain $\mathrm{k}_{\mathrm{p}}$, and integral gain $\mathrm{k}_{\mathrm{i}}$. The algorithm portions of the system are not shown but include PRBS injection, cross correlation of the input and output to obtain the impulse response estimate, and system identification, as shown in Figure 3.

The plant is a double pole plant with poles placed at $\mathrm{p}$, the closed-loop transfer function, including PID compensator and a double plant pole, takes the following form:

$$
C L(s)=\left(\frac{k_{d} s^{2}+k_{p} s+k_{i}}{s(s+p)^{2}+k_{d} s^{2}+k_{p} s+k_{i}}\right)
$$

In this case, $\mathrm{k}_{\mathrm{d}}$ is the derivative gain, $\mathrm{k}_{\mathrm{p}}$ is the proportional gain, $\mathrm{k}_{\mathrm{i}}$ is the integral gain, and $\mathrm{p}$ is the double plant pole location. The closed-loop transfer function is noted as CL(s). 
The test system utilized for this validation has a double plant pole at $\mathrm{s}=-3$, a double compensator zero at $\mathrm{s}=-15$, and a loop gain of 1 . A 15-bit PRBS with amplitude of 1 was injected into the system over a time period of 250 seconds to identify the system. Onehundred seconds of the output of the system, due to PRBS injection, is shown in Figure 24. The estimated impulse response is compared to the MATLAB impulse() function and is shown in Figure 25.

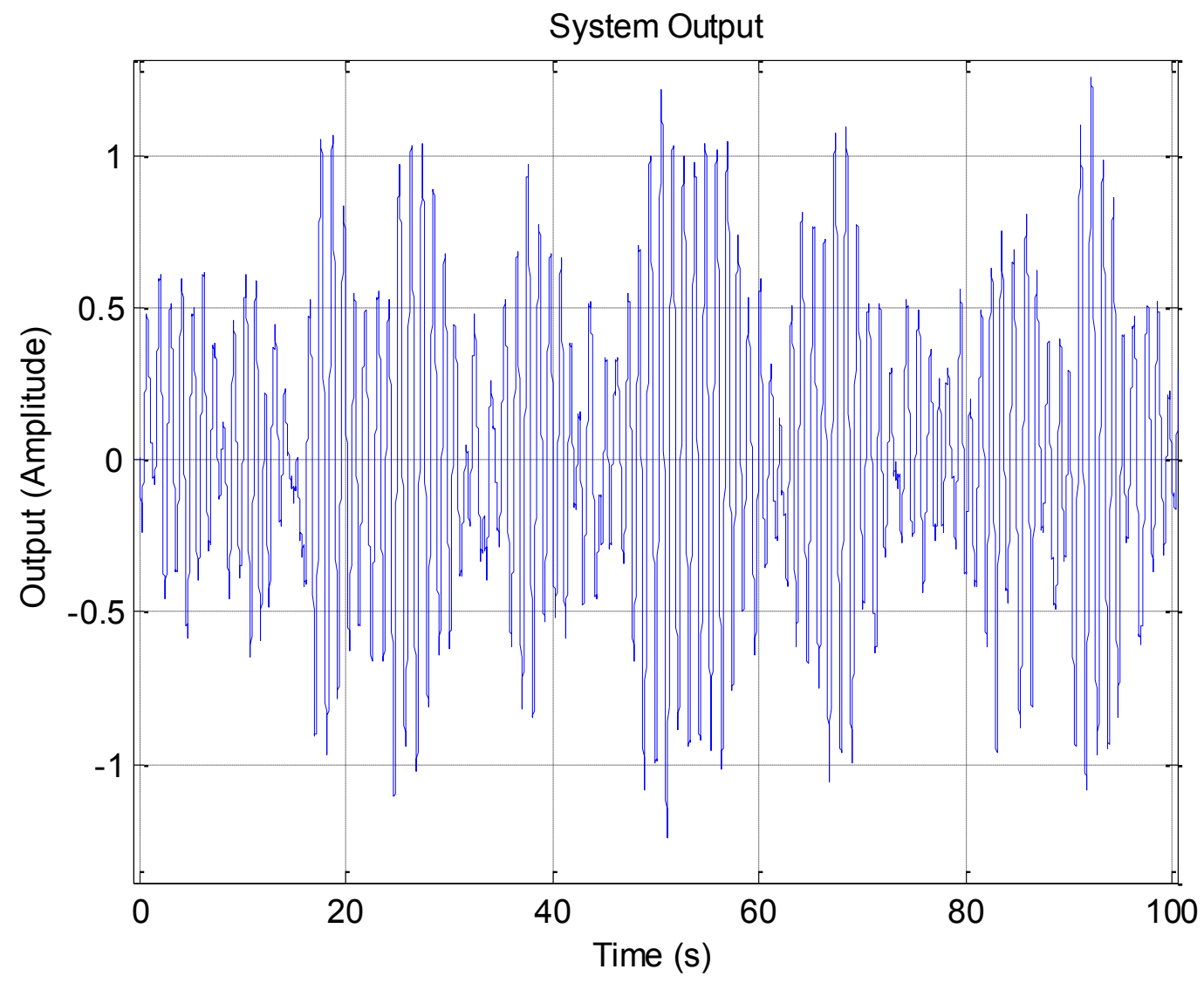

Figure 24 - System Output for Second-Order PID Compensated System With Zero TimeDelay Due to Pseudo-Random Binary Sequence Injection

This figure shows the system output when a 15-bit PRBS with an amplitude of 1 is injected into the test system. This system has a loop gain of 1 , a double compensator zero placed at $\mathrm{s}=-15$, a double plant pole at $\mathrm{s}=-3$, and time-delay set to zero. 


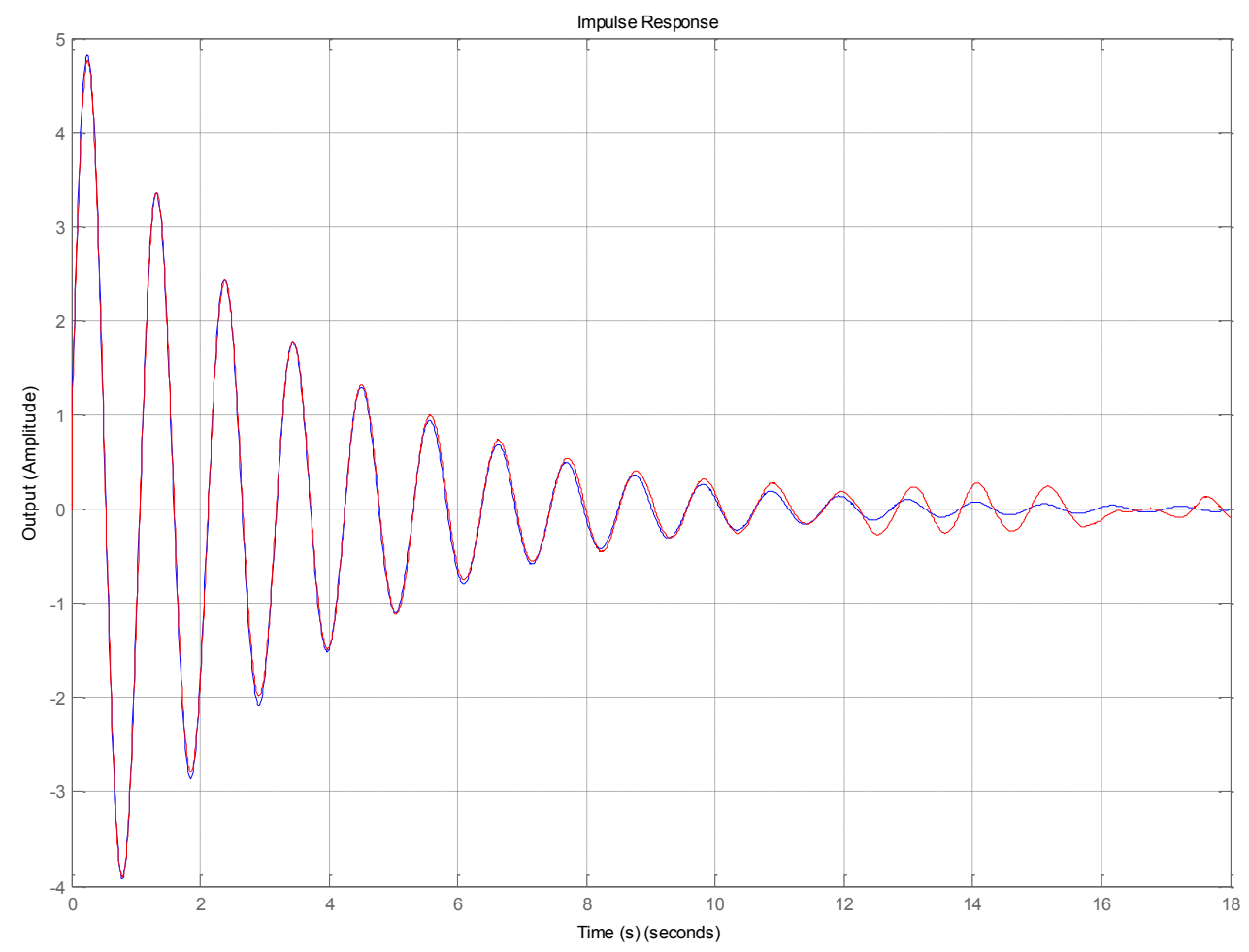

Figure 25 - Impulse Response Comparison Between MATLAB impulse() Function (blue) and Cross-Correlation Result (red) of a Second-Order PID Compensated System With Zero Time-Delay

This figure shows the estimated impulse response from the cross correlation output in red, compared to the impulse response from the MATLAB impulse() function in blue. This system has a loop gain of 1, a double compensator zero placed at $\mathrm{s}=-15$, a double plant pole at $\mathrm{s}=-3$, and time-delay set to zero.

The system identification routine identified the open loop plant poles at $\mathrm{s}=-2.9411$ with the actual plant poles being located at $s=-3$. This validates the use of PRBS injection, cross correlation of input and output to obtain the impulse response estimate, and the proper 
identification of the system using the MATLAB lsqcurevefit() function for a second-order system with no time-delay. The Bode plot of the frequency response is shown in Figure 26.
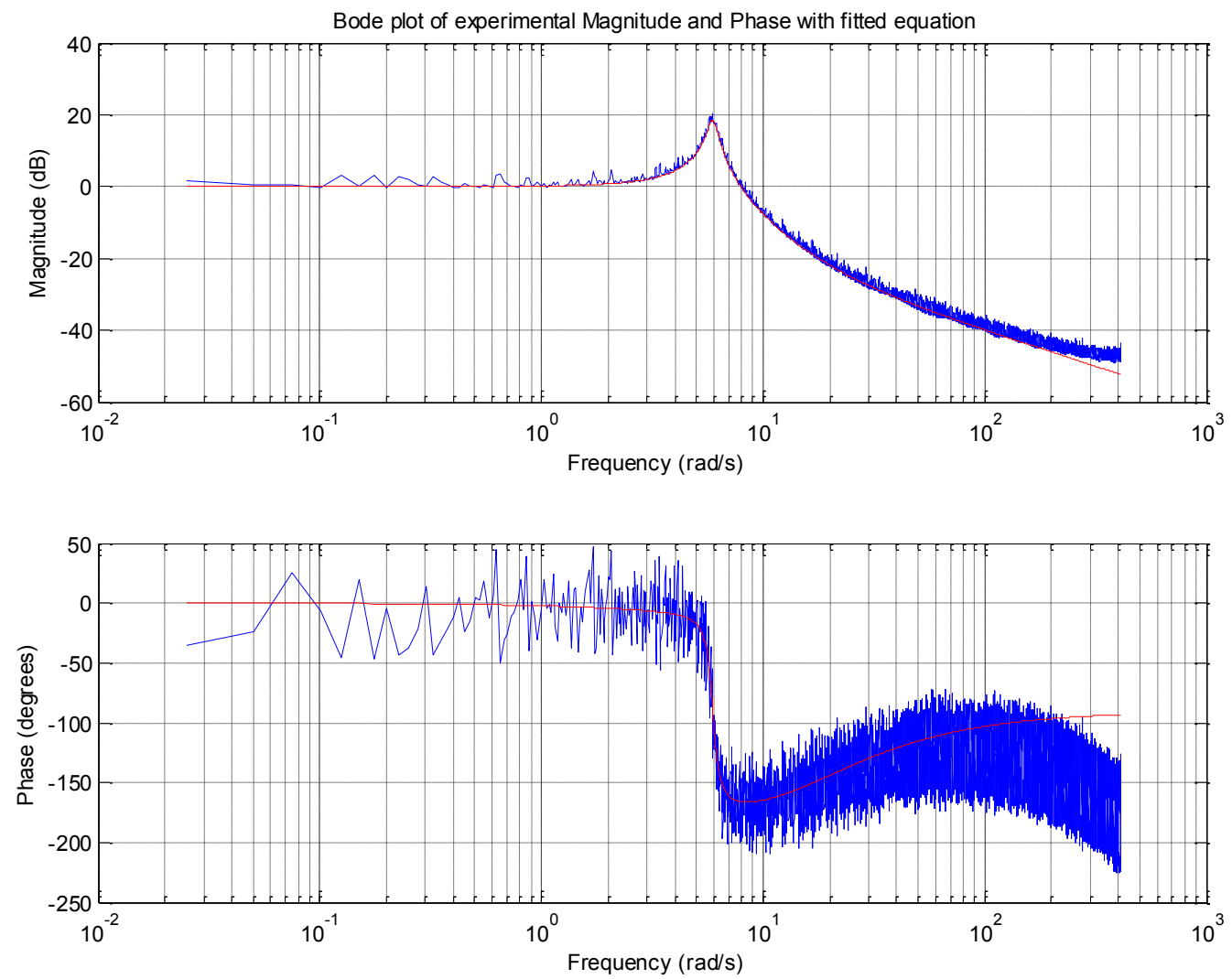

Figure 26 - Comparison of Bode Plot for Identified System (red) to Bode Plot Data from Cross-Correlation (blue) of a Second-Order PID Compensated System With Zero TimeDelay

This figure shows that the identified double plant poles at $\mathrm{s}=-2.9411$ match the actual double plant poles at $\mathrm{s}=$ -3. The identified system Bode plot is shown in red compared to the estimated frequency response data which is shown in blue. The close match is shown by the fact that the identified system response runs through the center of the estimated frequency response data. 
These data validate the algorithm for first and second-order systems with time-delay set to zero. A PRBS was injected into the system, the cross correlation of the input and output was performed, and the system was identified in both the first and second-order cases. 


\section{Results of Real-Time Auto Tuning Algorithm on Plants With Time-Delay}

This chapter focuses on the results of the algorithm when run on systems in the NTD region of 0.5 to 1 . The algorithm is first demonstrated using an NTD of 0.5 . This demonstration is followed by an example of the algorithm's ability to properly tune a timedelayed first and second-order system for NTD of 1.

\subsection{First-Order PI Compensated Plant With Normalized Time-Delay $=0.5$}

The first-order PI compensated plant with time-delay is shown in Figure 27. The remainder of the algorithm blocks including PRBS injection, cross correlation of input and output to obtain the impulse response estimate, system identification, gain calculation, and closed-loop pole placement have not been included in this figure but are shown in Figure 3.

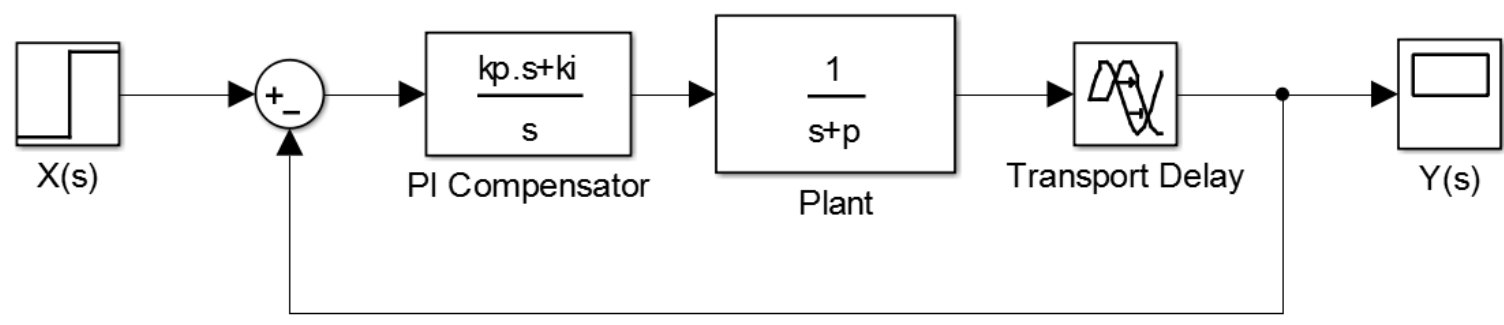

Figure 27 - First-Order PI Compensated Plant With Time-Delay

The plant is a single pole plant with plant pole at $\mathrm{p}$, proportional gain $\mathrm{k}_{\mathrm{p}}$, integral gain $\mathrm{k}_{\mathrm{i}}$. and time-delay $\mathrm{T}_{\mathrm{d}}$. The algorithm portions of the system are not shown but include PRBS injection, cross correlation of input and output to obtain the impulse response estimate, system identification, and gain calculation, as shown in Figure 3. 
The closed-loop transfer function of the system, including PI compensator, a single plant pole, and internal time-delay, is given as:

$$
C L(s)=\frac{k_{p}\left(s+\frac{k_{i}}{k_{p}}\right)}{e^{s T d} s(s+p)+k_{p} s+k_{i}}
$$

In Equation (10) the time-delay is defined as $T_{d}$, the plant pole is defined as $p$, the proportional gain is $\mathrm{k}_{\mathrm{p}}$, the integral gain is $\mathrm{k}_{\mathrm{i}}$, and the closed-loop transfer function is given as $\mathrm{CL}(\mathrm{s})$.

The test system utilized for this demonstration has a plant pole at $\mathrm{s}=-4$, a compensator zero at $\mathrm{s}=-5$, a loop gain of 1 , a time-delay of 0.125 seconds, and an NTD of 0.5. A 15-bit PRBS with an amplitude of 1 was injected into the system over a time period of 250 seconds to identify the system, assess the time-delay, place the compensator zeros and adjust the loop gain to achieve the commanded overshoot. One-hundred seconds of the output of the system due to PRBS injection is shown in Figure 28. The estimated impulse response is shown in Figure 29. 


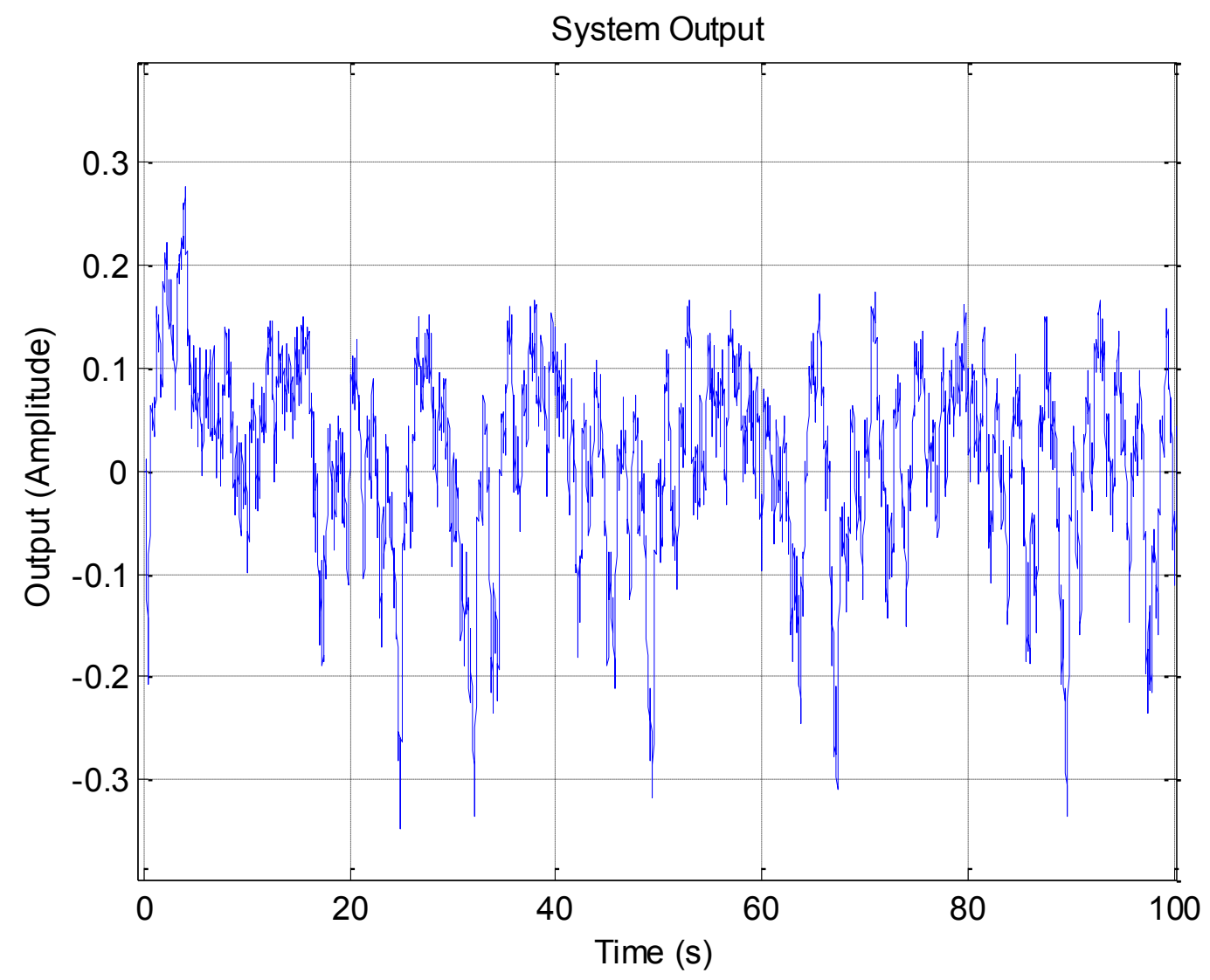

Figure 28 - System Output for First-Order PI Compensated System With Time-Delay Due to Pseudo-Random Binary Sequence Injection

This figure shows 100 seconds of the system output when a 15-bit PRBS with an amplitude of 1 is injected into the system. This system has a loop gain of 1 , a compensator zero placed at $s=-5$, a plant pole at $s=-4$, and a time-delay of 0.125 seconds. This delay corresponds to an NTD of 0.5 , since the plant time constant is 0.25 seconds. 


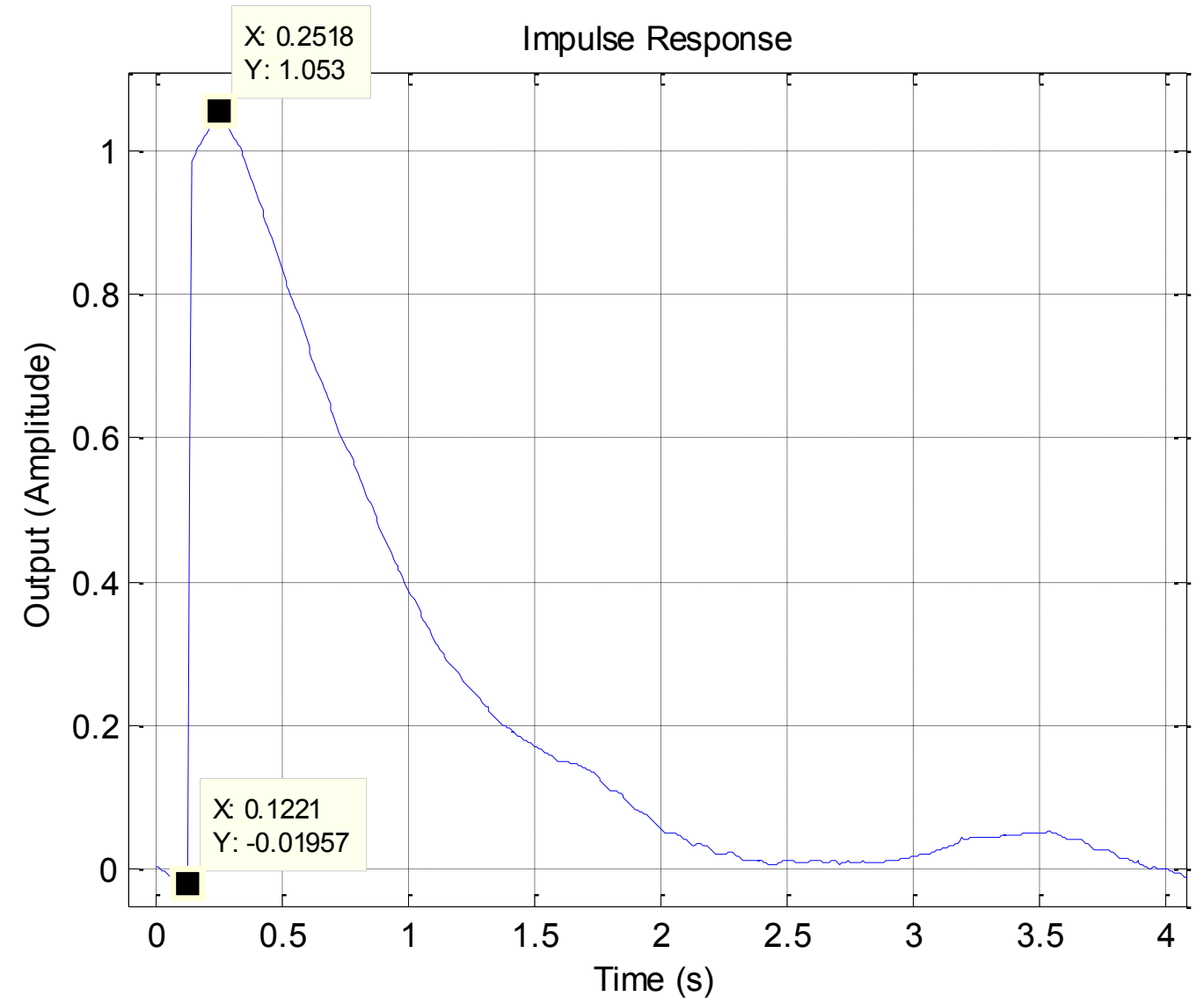

Figure 29 - Estimated Impulse Response of a First-Order PI Compensated System With Normalized Time-Delay $=0.5$

This figure shows the estimated impulse response from the cross correlation output. This system has a loop gain of 1 , a compensator zero placed at $\mathrm{s}=-5$, a plant pole at $\mathrm{s}=-4$, and a time-delay of 0.125 seconds. This timedelay corresponds to an NTD value of 0.5 . The estimated time-delay is evaluated at 0.1221 seconds by the algorithm.

The system identification routine identified the open loop plant pole at $\mathrm{s}=-3.811$ with the actual pole being located at $\mathrm{s}=-4$. This places the PI compensator zero at $\mathrm{s}=$ -4.1921. The estimated time-delay was found to be 0.1221 seconds with the actual time-delay being 0.125 seconds. The proper identification of the plant pole and the estimation of the time-delay validate the use of PRBS injection, the cross correlation of input and output to 
obtain the impulse response estimate, and the proper identification of the system using the MATLAB lsqcurevefit() function. The Bode plot of the estimated frequency response is shown in Figure 30.
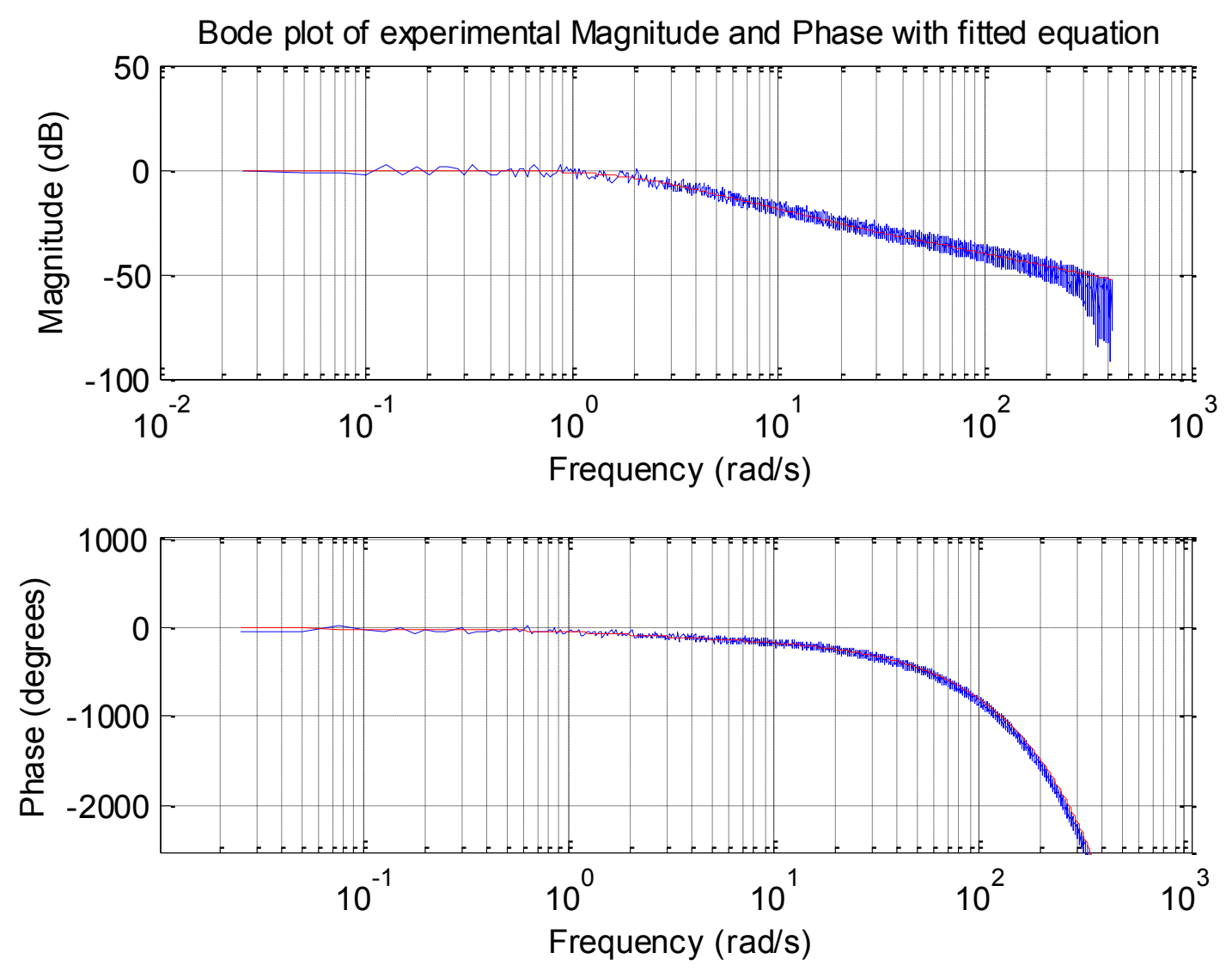

Figure 30 - Comparison of Bode Plot for Identified System (red) to Bode Plot Data from Cross-Correlation (blue) of a First-Order PI Compensated System With Normalized Time-Delay $=0.5$

This figure shows that the identified system pole at $\mathrm{s}=-3.811$ matches the actual system pole at $\mathrm{s}=-4$. The identified system Bode plot is shown in red compared to the estimated frequency response data which is shown in blue. The close match is shown by the fact that the identified system response runs through the center of the estimated frequency response data. The phase plot does not stabilize at a certain value but rolls off as frequency increases due to the time-delay exponential. 
The final step in the algorithm is to calculate the gain for the commanded overshoot. Figure 31 shows the system response for commanded overshoots utilizing the gain calculated for the identified system. With the compensator zero placed at $\mathrm{s}=-4.1921$, which corresponds to 1.1 times the identified plant pole, the step responses do not match the results shown in Figures 15 and 16 due to the offset compensator zero placement. The overshoots are slightly larger but are still within $5 \%$ of the commanded overshoot.

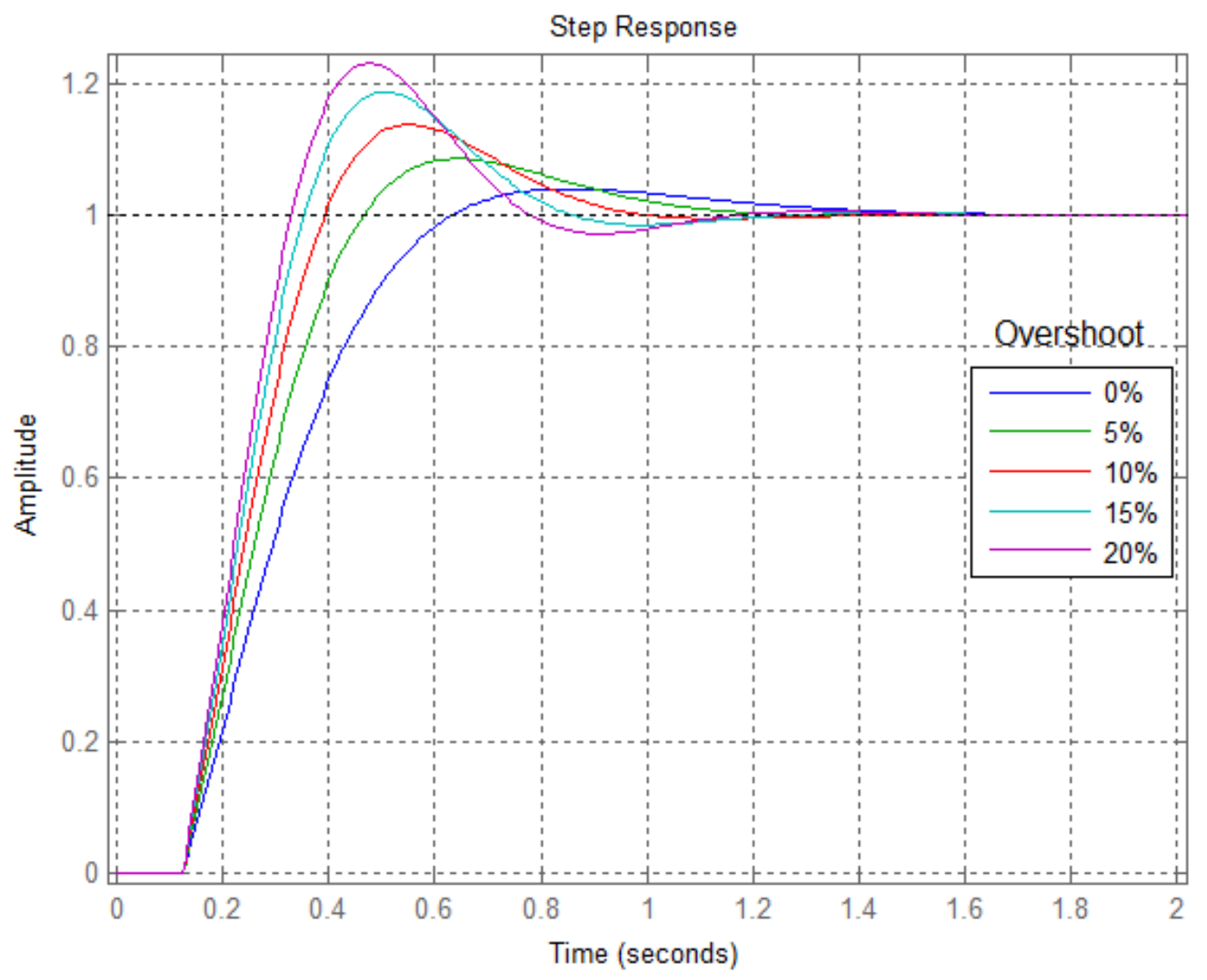

Figure 31 - Step Response Outputs for a First-Order PI Compensated Plant Tuned for Commanded Overshoots of 0, 5, 10, 15, and 20\%

System step response results after tuning for various commanded overshoots. It is important to note that the overshoots do not match the commanded overshoots seen in Figures 15 and 16 due to the error in the identification of the plant pole. The actual overshoots observed, however, match the commanded results within $5 \%$. 


\subsection{Second-Order PID Compensated Plant With Normalized Time-Delay $=0.5$}

The second-order PID compensated plant with time-delay is shown in Figure 32. The remainder of the algorithm blocks including PRBS injection, cross correlation of input and output to obtain the impulse response estimate, system identification, gain calculation, and closed-loop pole placement have not been included in this figure but are shown in Figure 3.

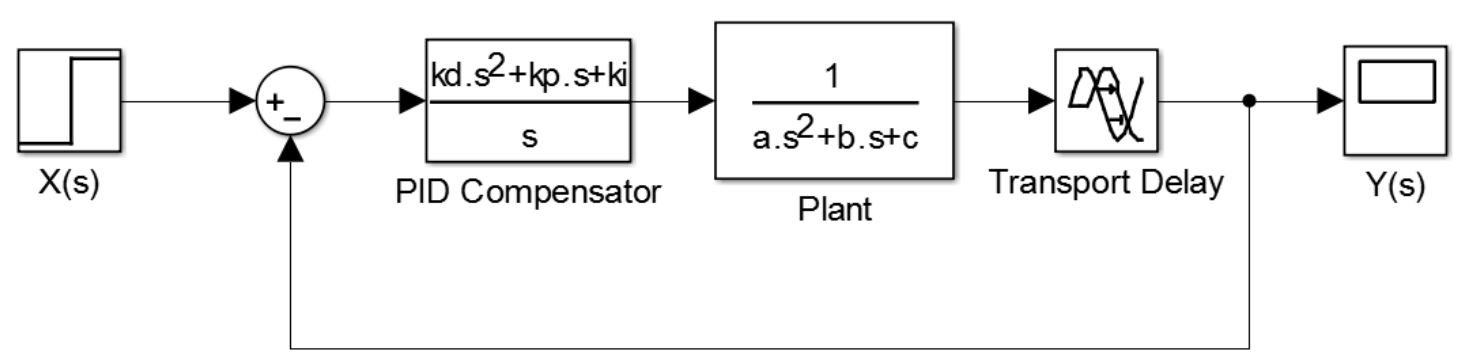

Figure 32 - Second-Order PID Compensated Plant With Time-Delay

Second-order PID compensated plant with an inherent time-delay. This plant utilizes a PID compensator with derivative gain $\mathrm{k}_{\mathrm{d}}$, proportional gain $\mathrm{k}_{\mathrm{p}}$, integral gain $\mathrm{k}_{\mathrm{i}}$, and a second-order plant. The second-order plant is shown in the standard form but analysis performed in this paper utilizes a double pole plant with plant poles at $\mathrm{s}$ $=-$ p. The time-delay is shown via a transport delay block.

The closed-loop system includes a double pole plant with pole location at $\mathrm{p}$, a PID compensator in the forward path, and inclusion of the time-delay exponential. The closedloop transfer function is given as:

$$
C L(s)=\left(\frac{k_{d} s^{2}+k_{p} s+k_{i}}{e^{s T} d s(s+p)^{2}+k_{d} s^{2}+k_{p} s+k_{i}}\right)
$$

The time-delay is defined as $T_{d}$, the derivative gain is defined as $\mathrm{k}_{\mathrm{d}}$, the proportional gain is defined as $\mathrm{k}_{\mathrm{p}}$, the integral gain is defined as $\mathrm{k}_{\mathrm{i}}$, the plant double pole is located at $\mathrm{p}$, and the system transfer function is defined as $\mathrm{CL}(\mathrm{s})$. 
The test system utilized for this demonstration has a double plant pole at $\mathrm{s}=-3$, a double compensator zero at $\mathrm{s}=-4$, a loop gain of 2 , a time-delay of 0.167 seconds, and an NTD of 0.5 . A 15-bit PRBS with an amplitude of 1 was injected into the system over a time period of 250 seconds to identify the system, assess the time-delay, place the compensator zeros and adjust the loop gain to achieve the commanded overshoot. One-hundred seconds of the output of the system due to PRBS injection is shown in Figure 33. The estimated impulse response is shown in Figure 34.

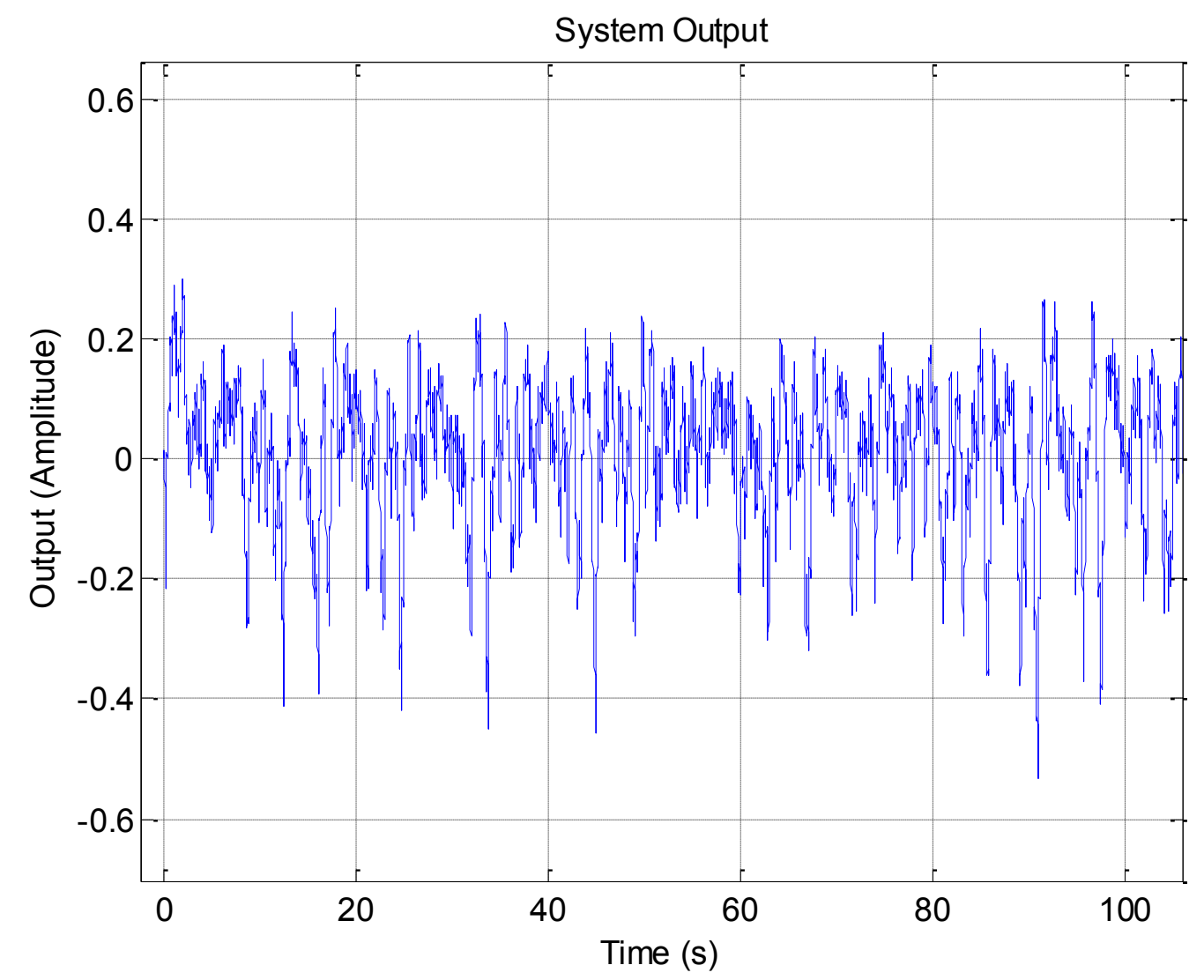

Figure 33 - System Output for Second-Order PID Compensated System With Time-Delay Due to Pseudo-Random Binary Sequence Injection

This figure shows the system output when a 15-bit PRBS with an amplitude of 1 is injected into the system. This system has a loop gain of 2 , a double compensator zero placed at $\mathrm{s}=-4$, double plant poles at $\mathrm{s}=-3$ and a time-delay of 0.167 seconds. This corresponds to an NTD of 0.5 . 


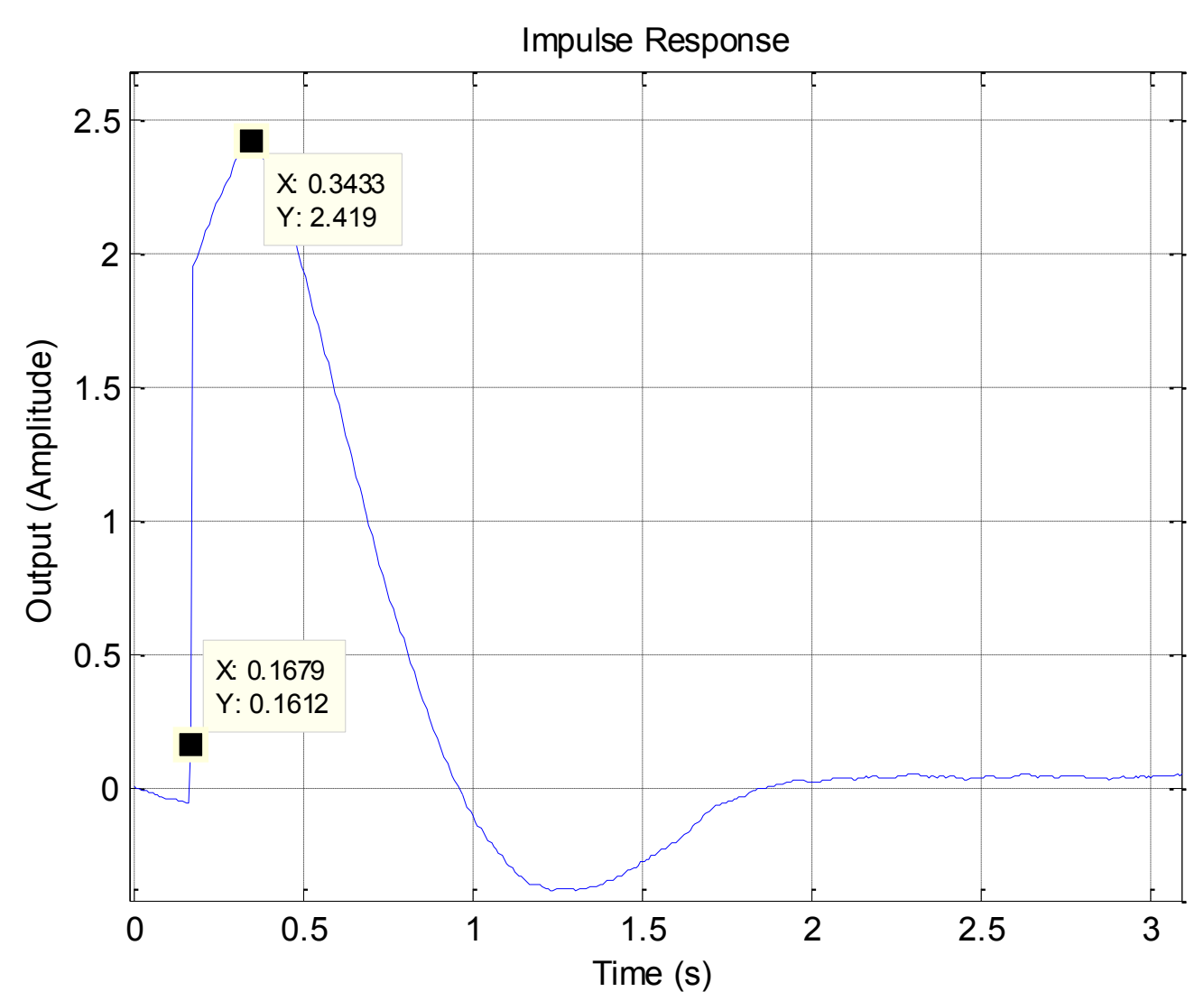

Figure 34 - Estimated Impulse Response of a Second-Order PID Compensated System With Normalized Time-Delay $=0.5$

This figure shows the estimated impulse response from the cross correlation output. This system has a loop gain of 2, a double compensator zero placed at $\mathrm{s}=-4$, a double plant pole at $\mathrm{s}=-3$, and a time-delay of 0.167 seconds. This time-delay corresponds to an NTD value of 0.5 . The estimated time-delay is evaluated at 0.1679 seconds by the algorithm.

The system identification routine identified the open loop double plant poles at $\mathrm{s}=-2.9277$ with the actual double plant poles being located at $\mathrm{s}=-3$. The estimated time-delay was evaluated as 0.1679 seconds compared to an actual time-delay of 0.167 seconds. This validates the use of PRBS injection, cross correlation of input and output to obtain the impulse response estimate, and the proper identification of the system using the MATLAB 
lsqcurevefit() function for a second-order system with time-delay. The Bode plot of the frequency response is shown in Figure 35.
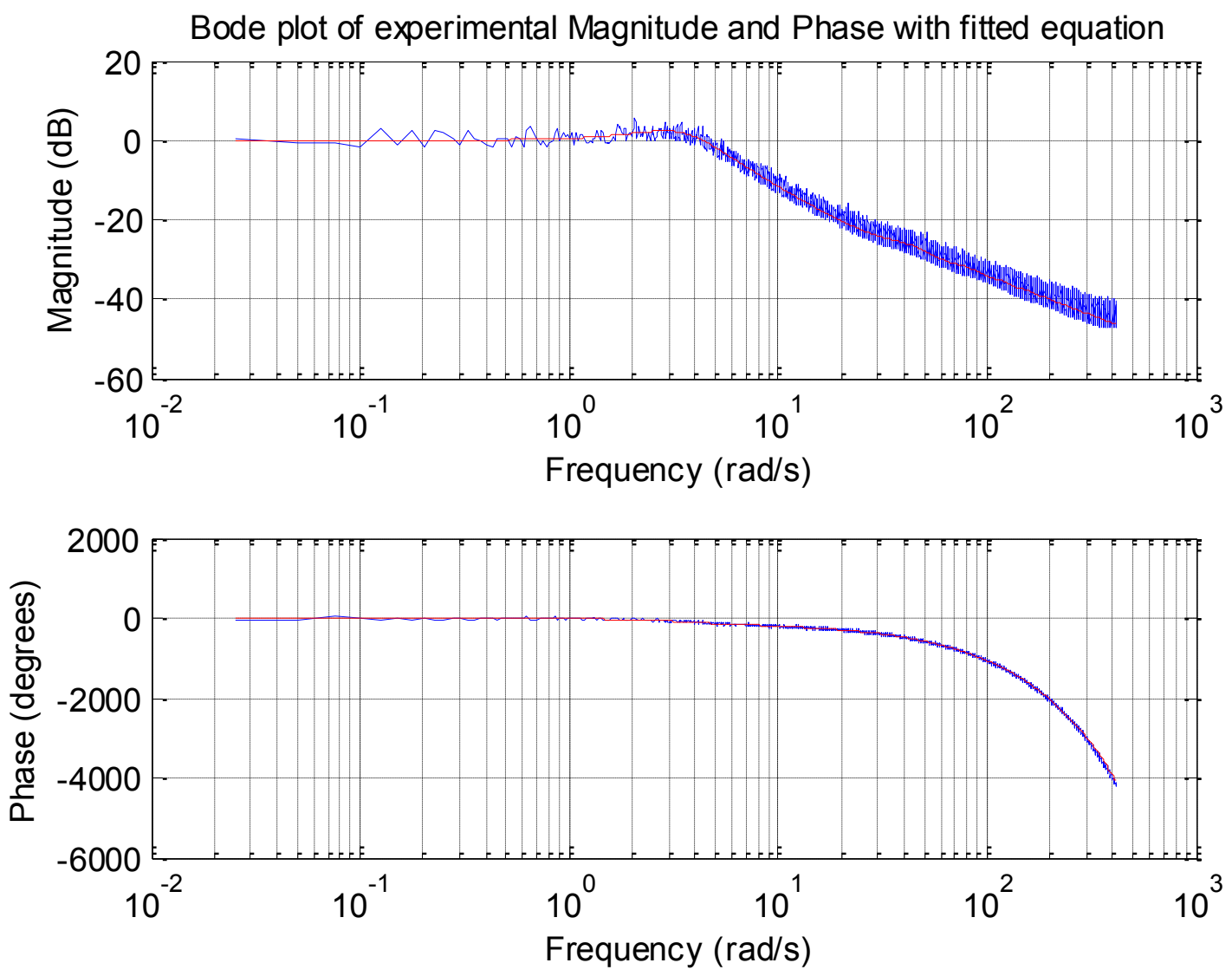

Figure 35 - Comparison of Bode Plot for Identified System (red) to Bode Plot Data from Cross-Correlation (blue) of a Second-Order PID Compensated System With Normalized Time-Delay $=0.5$

This figure shows that the identified system pole at $\mathrm{s}=-2.9277$ matches the actual system pole at $\mathrm{s}=-3$. The identified system Bode plot is shown in red compared to the estimated frequency response data which is shown in blue. The close match is shown by the fact that the identified system response runs through the center of the estimated frequency response data. The phase plot does not stabilize at a certain value but rolls off as frequency increases due to the time-delay exponential.

Since the system is identified as a double pole plant with $\mathrm{s}=-2.9277$, the PID compensator zeros are placed slightly off, at $\mathrm{s}=-3.2205,-2.6349$. This is evidenced in the 
slightly lower overshoots seen in the output responses compared to the ideal results seen in

Figures 17 and 18. In this case, all overshoot results are within 5\% of the commanded values as shown in Figure 36.

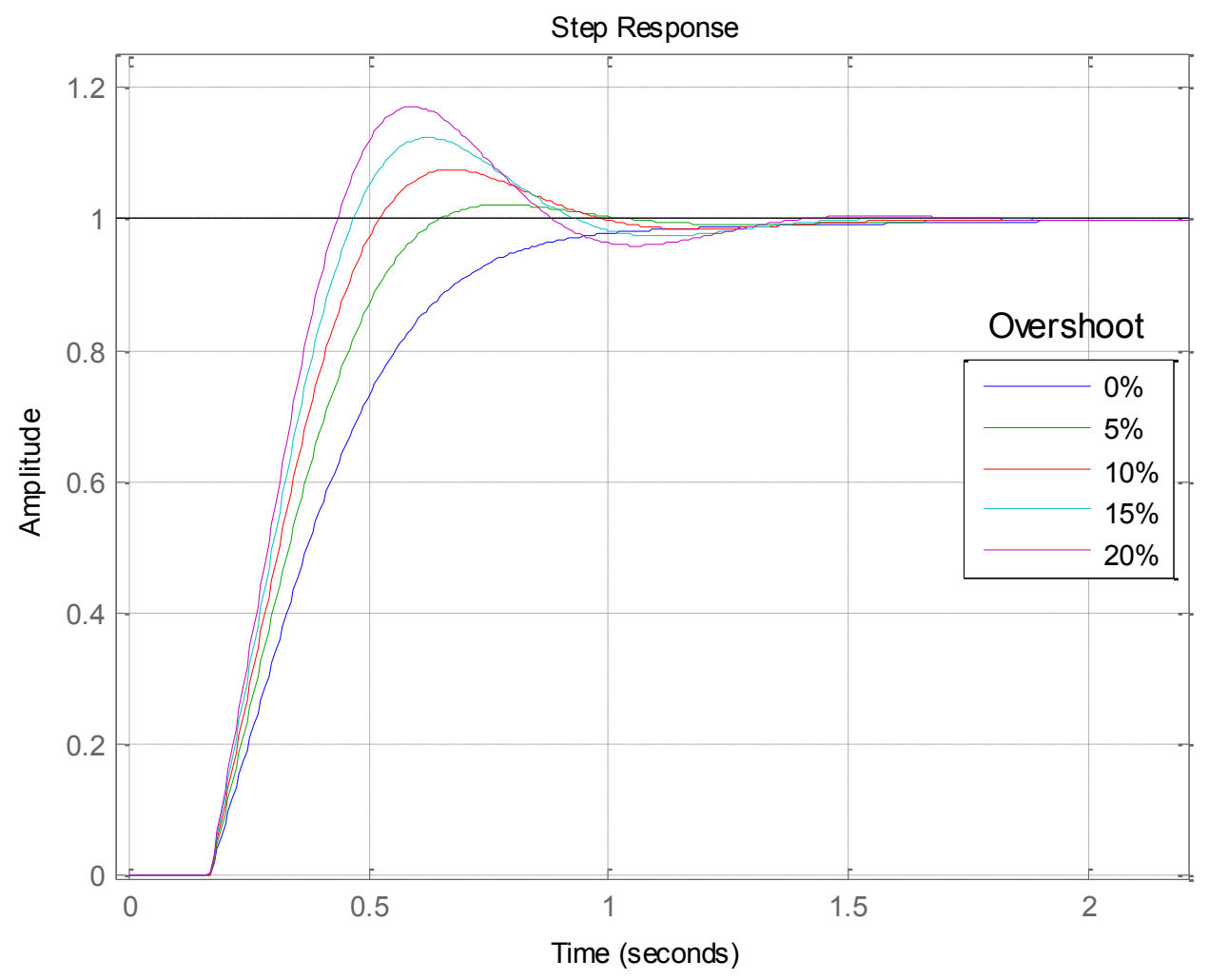

Figure 36 - Step Response Outputs for a Second-Order PID Compensated Plant Tuned for Commanded Overshoots of 0, 5, 10, 15, and 20\%

System step response results after tuning for various commanded overshoots. It is important to note that the overshoots do not match the commanded overshoots seen in Figures 17 and 18 due to the error in the identification of the double plant pole. The actual overshoots observed, however, match the commanded results within $5 \%$. 


\subsection{First-Order PI Compensated Plant With Normalized Time-Delay $=1$}

As a justification for this method it is important to look beyond what has been done in previous work (Baker, 2011). For NTD $>0.5$, auto tuning has not been adequately explored. In a situation like this it is easy to come up with an example of why a system like this is important to understand. For example, if there was a well tuned system that was stable at a specific operating condition, it could become unstable with a slow drift in time-delay. The auto tuning algorithm would be able to ensure the system remained stable and adjust the performance to maintain the commanded overshoot.

For example, the system from the first-order results section, with a plant pole of $\mathrm{s}=$ -4 , is tuned using the auto tuning algorithm and exhibits a typical response of approximately $10 \%$ overshoot after tuning. If the time-delay inherent in the system is increased, from NTD $=0.5$ to 1 , the system dynamics will change and re-tuning will need to occur for proper operation. In this case the time-delay increase, leads to oscillatory behavior, as shown in Figure 37.

By re-applying the tuning algorithm to the system, it may no longer be possible to achieve a response that is comparable to the plant pole response, but at least the system will be tuned to be stable and maintain an overshoot that meets the design specifications of the system. By re-applying the auto-tuning method, the acceptable response shown in Figure 38 is achieved.

This result shows that the system can be maintained in a properly tuned state as timedelay is changed. This result shows that a system can still be stabilized with an optimum performance given a large time-delay that is on the order of the plant time constant. With 
previous methods, this compensator would have to be re-designed or tuned to accommodate the added delay. This auto tuning behavior could prevent hazardous situations where a system could be damaged or cause unexpected behavior from a time-delay shift.

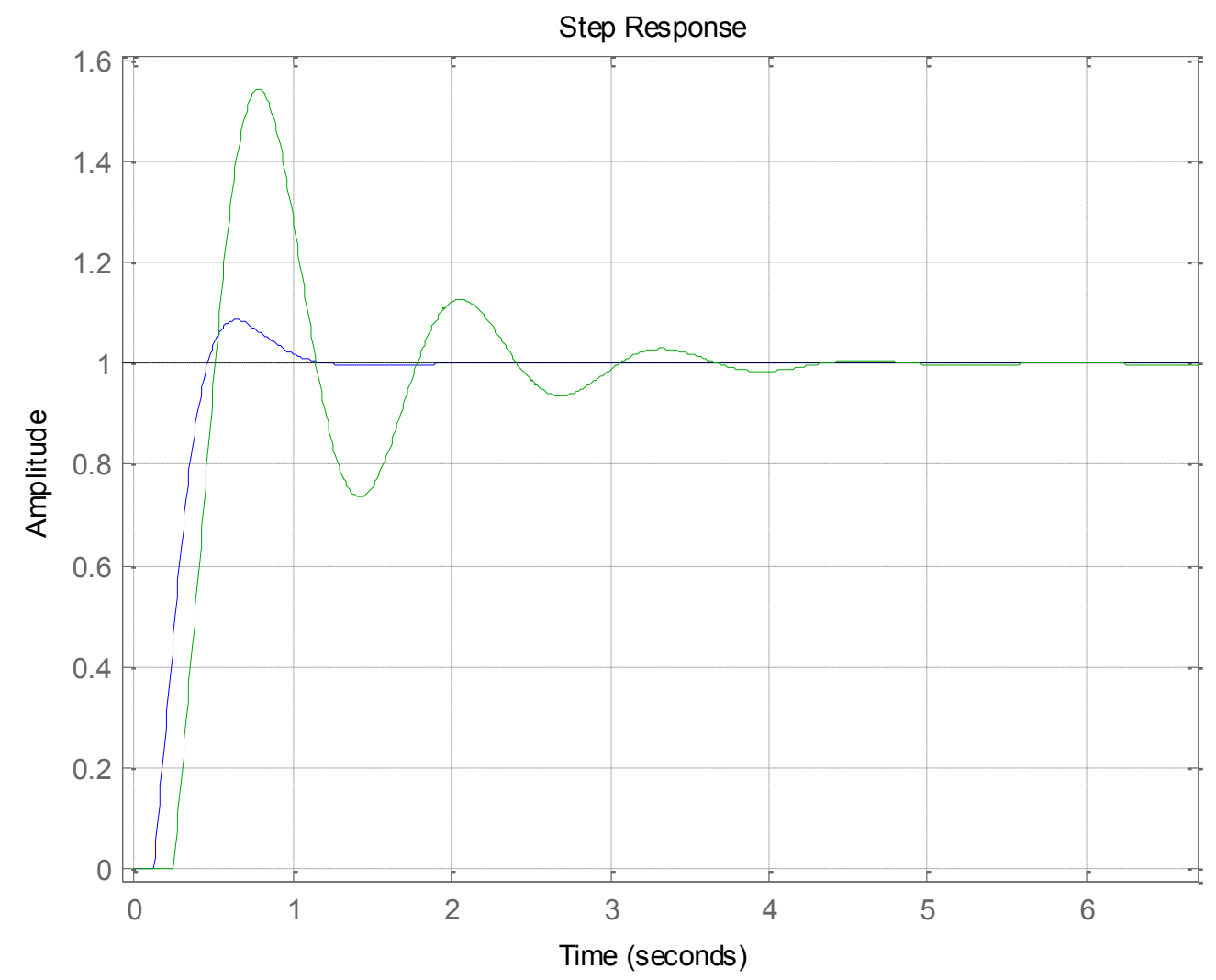

Figure 37 - First-Order PI Tuned Step Response for Normalized Time-Delay =1 (blue) and De-Tuned Response (green)

This figure shows an initially tuned system with a plant pole at $\mathrm{s}=-4$ and a compensator zero placed at $\mathrm{s}=-4.4$. This system was originally tuned using the algorithm in this paper to achieve an overshoot of $10 \%$ for an NTD of 0.5 , shown in blue. The time-delay was then increased to 0.25 seconds which creates an NTD of 1 . This causes oscillatory behavior outside the $10 \%$ overshoot specification, as shown by the green response. 


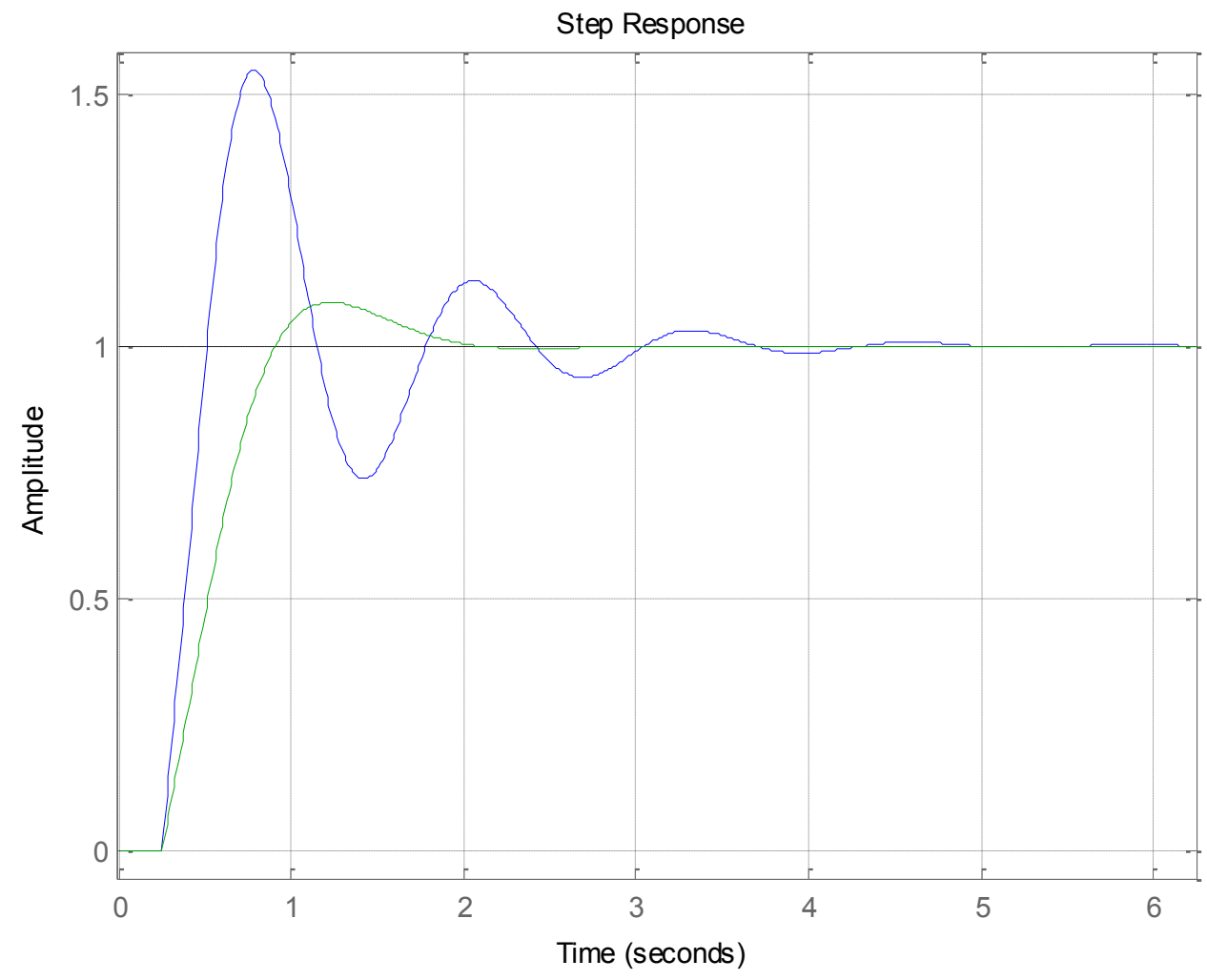

Figure 38 - First-Order PI Compensated System With Oscillatory Response (blue) and ReTuned Response (green)

This figure shows the oscillatory response from the previous figure in blue, which was a response for the detuned system at an NTD of 1 . This green response shows the response after re-tuning which matches the original overshoot by sacrificing the time response. 


\subsection{Second-Order PID Compensated Plant With Normalized Time-Delay $=1$}

The second-order PID compensated Plant can be shown to exhibit similar behavior when a larger time-delay is introduced. In this case, a second-order plant with double poles at $\mathrm{s}=-3$ is utilized to demonstrate the algorithm. This plant has been previously compensated utilizing the real-time auto tuning algorithm and exhibits the behavior shown in Figure 39. This figure shows the plant as tuned for an NTD of 0.5 and the de-tuned plant with an NTD of 1 . As was seen in the last section, it is possible to re-tune the plant by running it through the auto tuning algorithm. Figure 40 shows similar results to the first-order plant in that the system overshoot is reduced with a slight sacrifice in the system speed. 


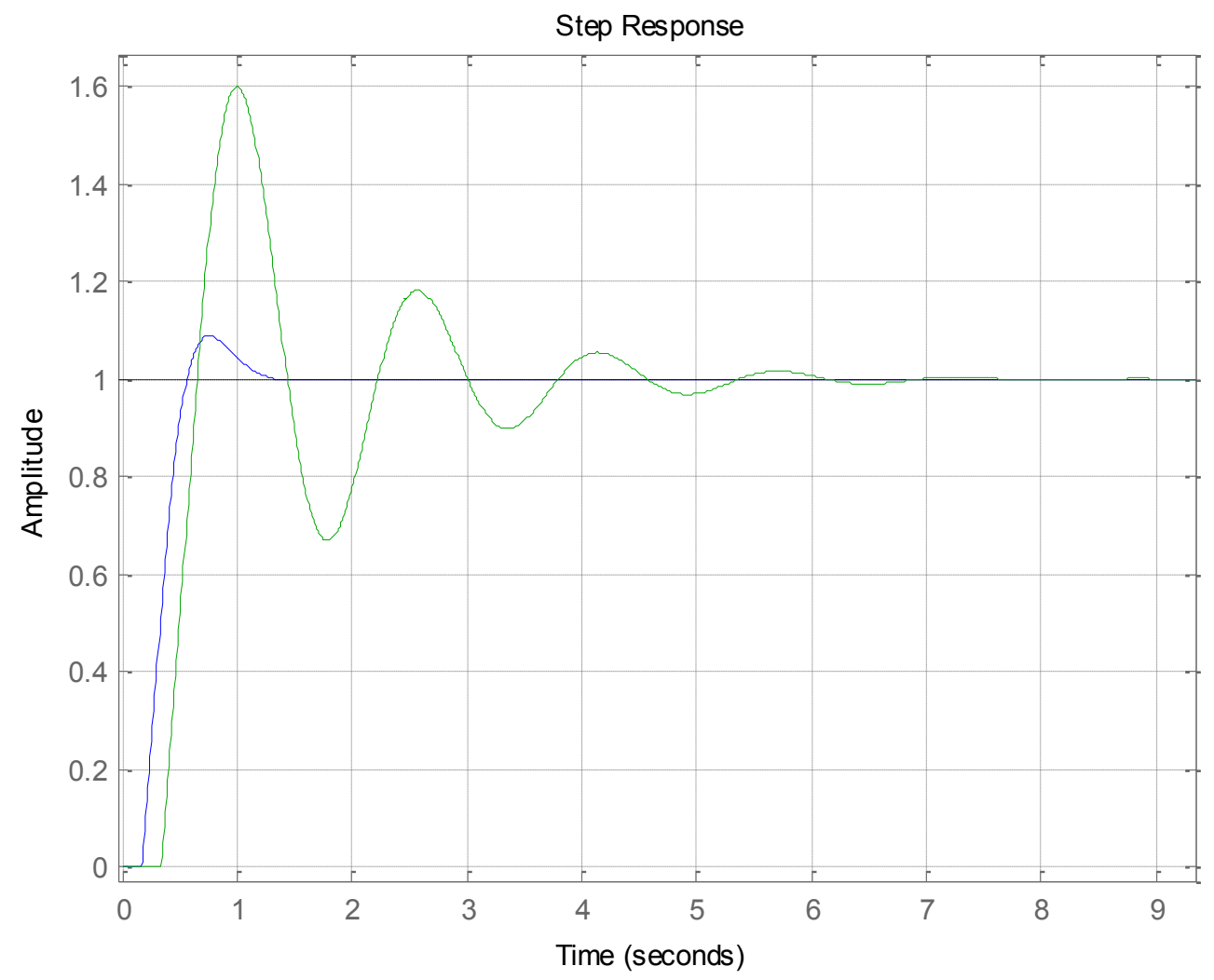

Figure 39 - Second-Order PID Tuned Step Response for Normalized Time-Delay = 1 (blue) and De-Tuned Response (green)

This figure shows the originally tuned plant response for a second-order system with double plant poles at $\mathrm{s}=-3$ in blue. By increasing the time-delay to 0.33 seconds, corresponding to an NTD of 1 , the plant exhibits oscillatory behavior due to the time-delay, as shown in green. 


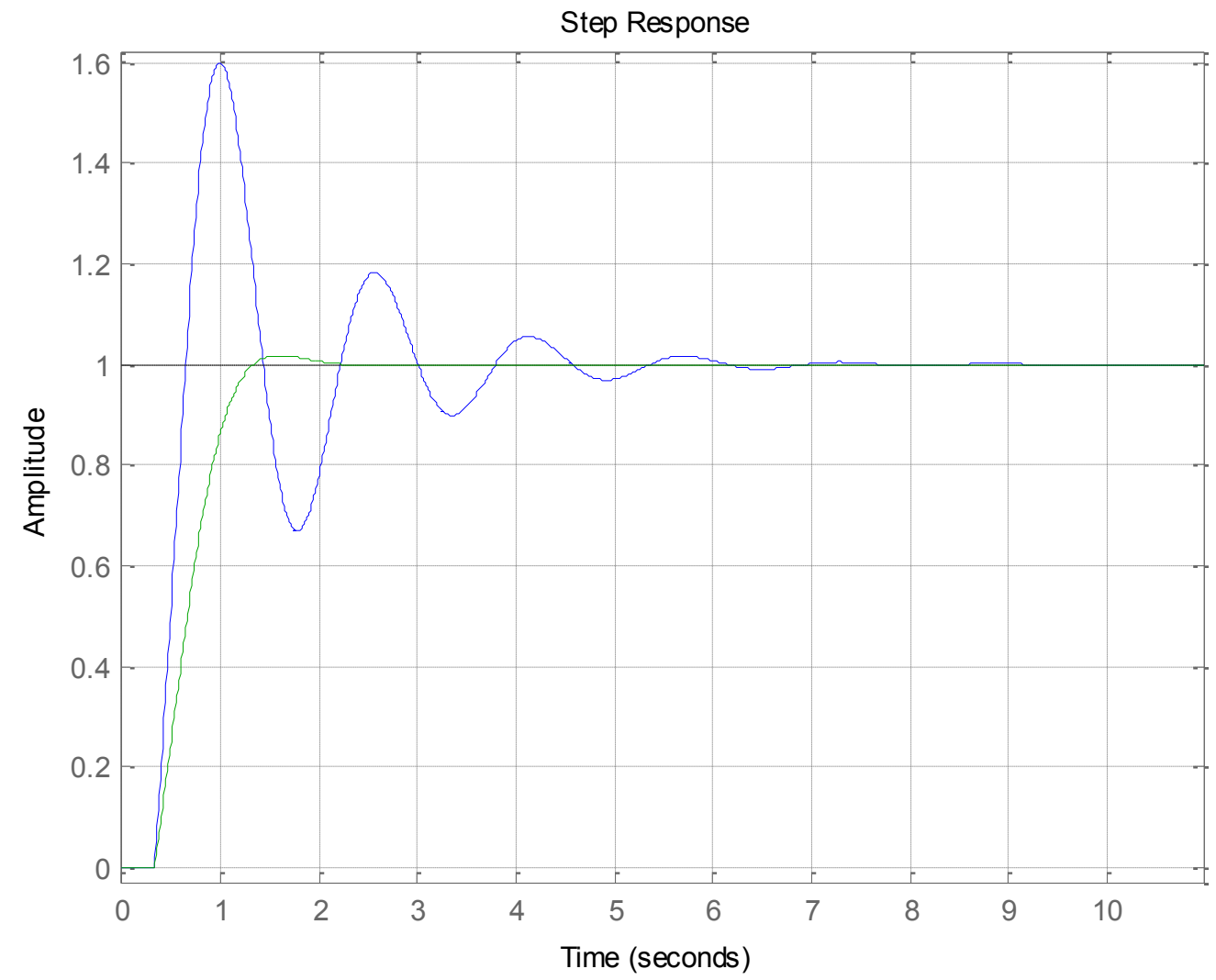

Figure 40 - Second-Order PID Compensated System With Oscillatory Response (blue) and Re-Tuned Response (green)

This plot shows a second-order PID compensated plant response which has been de-tuned by increasing the time-delay to 0.33 seconds, corresponding to an NTD of 1 , shown in blue. The plant is then re-tuned using the algorithm discussed in this work, shown in green, by sacrificing plant time response to achieve an overshoot reduction. 


\section{Conclusion}

This paper presents the results of a real-time auto tuning method for first and secondorder systems with time-delay using PRBS and the cross-correlation method. A PRBS was used to excite the dominant modes of the system to produce an estimate of the impulse response via the cross correlation of the input PRBS with the output of the closed-loop system. The estimate of the impulse response contains the dominant dynamics of the closedloop system and can be used to develop an estimate of the system's closed-loop transfer function and the time-delay. The time-delay is estimated using the maximum point in the derivative of the estimated impulse response. The FFT of the impulse response is taken to obtain an estimate of the frequency response of the system. This result is compared to the known form of the closed-loop transfer function for a first or second-order system with timedelay. The estimated frequency response data is compared to a model function using the MATLAB lsqcurvefit() function, which varies the plant pole location until it finds a minimum in the mean square error between the data and model function. This method utilizes a single search gradient descent method and an optimization function, that takes into account the current PID parameters, the estimated time-delay, and the appropriate form for the closed-loop transfer function. The estimated plant pole location and estimated time-delay are then used for compensator placement based on iterative tuning results shown in Tables 1 and 2 and the desired overshoot for the system.

The PRBS and cross-correlation portion of this real-time auto tuning algorithm required a decision about which size of PRBS to use and the length of time data that would 
be used for analysis. The longer the set of time data that the PRBS is being applied over, the more accurate the impulse response estimate will be. As the length of the time data gets shorter, the cross-correlation process will suffer from more artifacts in the system output which leads to a difficult system to analyze. The size of PRBS attempts to balance these competing factors to provide an acceptable time resolution and frequency resolution, while maximizing the accuracy of the estimated impulse response. The size of the PRBS was chosen as a 15-bit generator applied over a 250 second set of data, which provides for a time resolution of 7.6 milli-seconds and frequency dynamics up to $411.74 \mathrm{rad} / \mathrm{sec}$. In this range, there are still artifacts from the cross correlation process but the system is still identifiable with a relatively short data sample. These parameters could be adjusted to apply the method to a different range of systems by changing the number of bits in the PRBS or the length of time data utilized. The results in this research show that the PRBS frequency content was sufficiently rich to properly excite the example systems and achieve an accurate estimate of the impulse response and time-delay.

The system identification routine, utilized in this algorithm, was a MATLAB function that provides curve fitting using a Gradient Descent regression technique. The lsqcurvefit() function was used to compare the estimated frequency response data to the model transfer function and adjusted the plant pole parameter until the best fit was found. This comparison utilized a minimization of the mean square error based on an initial starting point determined by experimentation. It was found that the starting point for the regression technique needed to be large compared to the plant pole, so it was chosen above the frequency range that the system was designed to analyze. This ensures that the descent along the mean square error 
curve has the best chance of converging to the global minimum, and minimizes the chance that a local minimum would be found instead. The system identification routine was able to estimate the plant poles reliably and provide these poles to the compensator tuning portion of the algorithm.

The final portion of the algorithm involved the gain calculation based on a consistent compensator zero placement. By starting with the PID tuning method proposed by Baker (2011) a modified compensator zero placement method was developed to achieve a specific overshoot, based on the system time-delay. By consistently placing the compensator zeros at 1.1 times the plant pole for a first-order system, and 1.1 and 0.9 times the plant pole for a second-order system, the root locus generated using the time-delay exponential showed consistent behavior for each NTD value. This result was leveraged by iteratively tuning test systems to achieve a specific overshoot and relationships between overshoot, time-delay, and loop gain were developed. Tables 1 and 2 provide these relationships and are utilized in the auto tuning algorithm to reliably place the closed-loop poles to achieve a user commanded overshoot. The results of these relationships provide auto tuning results for an identified system within $5 \%$ of the commanded overshoot.

The results of this research demonstrate that the real-time auto tuning algorithm for a first or second-order system with time-delay is a viable method. The MATLAB routines that were written to simulate the behavior of the system and the results of each piece of the algorithm worked independently, as shown in the development section. They also worked together, as shown in the validation and results sections. The algorithm results were 
consistent with the goal of tuning the system to within $5 \%$ of the commanded overshoot and show that PRBS injection for system identification and automatic tuning of a time-delayed system is possible. 


\section{Discussion}

This research was a first step in the development of a real-time auto tuning algorithm for a first or second-order system with time-delay, but there are a few issues that warrant further investigation and improvement. This study was theoretically based and all results were obtained utilizing MATLAB simulation software. In real systems, there are many other aspects that need to be taken into account, including

- System noise

- Disturbance rejection

- Accuracy of system identification

- Algorithm tradeoffs

- Hardware implementation

Many of these issues have been addressed in previous research, but the ideas need to be consolidated and applied to the real-time auto tuning algorithm presented in this paper.

System noise is inherent in all systems due to the use of electronic amplifiers, computers, and the heating up of system components. With respect to the way that time-delay and noise interact, research has been conducted by Yue and Han (2005) that introduces important points which could be incorporated into the algorithm to make it more robust. Noise could also be reduced by averaging techniques that utilize multiple periods of PRBS signals, with different starting seed values, as demonstrated by Sohrab, Reischl, Grodins, and Yamashiro's application to respiratory systems (1978). This process takes longer to gather 
the data, but it will effectively reduce noise in the output as the output sequences are averaged together.

The next issue that was not addressed in this research is the disturbance rejection ability of the tuned system. The tuning of the system to achieve a desired overshoot does not take into account what will happen if there is a load disturbance applied to the system. The overshoot in the response to a step input may be adequate but there are tradeoffs that need to be made to balance the step response and the load disturbance rejection. The system may be well-tuned with respect to an overshoot for a step response but a load change may show a much larger response, which could cause undesirable excursion from the setpoint and possible damage to the system. In Baker's approach to compensator tuning (2011) these issues were discussed and could be applied in the future application of this algorithm.

The largest weakness of the algorithm was the identification of the plant poles of the system. Even a small error in the estimated plant pole location may cause the compensator zeros to be placed inappropriately for the system, which will lead to an offset in the commanded overshoot observed in the step response. This behavior is shown in the differences between figures 31 and 15 for a first-order system with time-delay, and figures 36 and 17 for a second-order system with time-delay. This result could be improved by performing a multiple starting point gradient descent technique and averaging the results, or by creating a smarter search algorithm that was customized to the application. By enhancing the implementation of the system identification algorithm, the identification results could be 
greatly improved, which would directly translate to more accurate compensation performance.

There was some comparison performed between the Baker method (2011) of compensator placement and the method proposed in this paper. However, there was not a discussion as to whether one method was preferred over the other for specific NTD situations. As was mentioned in this paper, the Baker method provides inferior results for NTD greater than 0.5. However, the Baker method may provide better results for NTD $<0.5$. The goal of this paper was not to explore an optimized algorithm but to present new results for a broader range of NTD than had been previously addressed. An important next step to the development of an optimized algorithm would be to analyze the tradeoffs between different compensation methods and include decision making points in the algorithm to use the method that is most optimal for the situation. The Baker method and the method explored in this paper are only two compensation methods that have been explored so it is also important to experiment with other compensation techniques that could yield better results.

The hardware implementation of this real-time auto tuning system is another issue that was not adequately discussed in this research. The algorithm claims to be a real-time auto tuning algorithm but, in reality, it is not feasible to adjust the gain for every new data sample taken. The calculations could be performed in real-time to produce a real-time gain recommendation but it would be impractical to update the compensator values in real-time. Updating the gain value continuously could even cause system instability if the compensator was not placed appropriately due to a cascade effect from the gain changes. This algorithm is 
real-time in a buffered sense because an adequate data sample needs to be collected before the first gain calculation can be run. The system could be continuously updating the cross correlation result, running the system identification routine, and calculating the required loop gain but there would need to be decision logic added to decide when to update the compensator locations.

This research validates the feasibility of the proposed method and the algorithm meets the overshoot performance specified. There are still improvements that can be made and issues that need to be addressed for further optimization and development. 


\section{References}

Astrom, K. J., \& Hagglund, T. (1995). PID controllers: Theory, design, \& tuning. Research Triangle Park, N.C.: International Society for Measurement and Control.

Baker, G. (2011). PID-Tuning of plants with time delay using root locus. (Master's thesis). Retrieved from SJSU ScholarWorks. (Paper 4036).

Bahill, A. T. (1983). A simple adaptive smith-predictor for controlling time-delay systems. Control Systems Magazine, IEEE, 3(2), pp 16-22. doi:10.1109/MCS.1983.1104748

Frazzoli, E. (2010). Time Delays. Retrieved from http://ocw.mit.edu/courses/aeronautics-andastronautics/16-30-feedback-control-systems-fall-2010/recitations/

MIT16_30F10_rec11.pdf

Isermann, R., \& Münchhof, M. (2011). Identification of dynamic systems: An introduction with applications. Heidelberg, N.Y.: Springer.

Miao, B., Zane, R., \& Maksimovic, D. (2004). A modified cross-correlation method for system identification of power converters with digital control. Power Electronics Specialists Conference, IEEE, 5, pp 3728-3733. doi:10.1109/PESC.2004.1355134

Ogata, K. (2002). Modern control engineering (4th ed.). Upper Saddle River, N.J.: Prentice Hall.

Oppenheim, A. V., \& Schafer, R. W. (2010). Discrete-time signal processing. Upper Saddle River: Pearson.

Sipahi, R., Niculescu, S., Abdallah, C., Michiels, W., \& Gu, K. (2011). Stability and stabilization of systems with time delay. Control Systems, IEEE, 31(1), pp 38-65. doi:10.1109/MCS.2010.939135

Sohrab, S., Reischl, P., Grodins, F. S., \& Yamashiro, S. M. (1978). Application of pseudo random binary sequence input to identification of respiratory control dynamics. Decision and Control, IEEE, 17, pp 1304-1308. doi:10.1109/CDC.1978.268130 
Vajta, M. (2000). Some remarks on Padé-Approximations. 3rd Tempus-Intcom Symposium, Vezprem, Hungary, 1-6.

Yue, D., \& Han, Q. (2005). Delay-Dependent exponential stability of stochastic systems with time-varying delay, nonlinearity, and markovian switching. Automatic Control, IEEE Transactions, 50(2), pp 217-222. doi:10.1109/TAC.2004.841935

Ziegler, J. G., \& Nichols, N. B. (1942). Optimum settings for automatic controllers. Transactions of the ASME, pp 759-765. 


\section{Appendix A}

\section{Pseudo-Random Binary Sequence Generation for Real-Time Auto Tuning}

This thesis required extensive use of PRBS for testing and development of the auto tuning method. The built in functions from MATLAB would have provided similar results, but to tie in with the real-time auto tuning process more fully and to allow for ease of testing, a function for generation was developed to support the project. This function includes the ability to choose specific tap configurations and choose the size of the PRBS which allows for customization of the sequence for specific scenarios. The function also allows the choice of the starting register values which provides for the ability to use the same length PRBS multiple times in a row to average out system noise in a real world application. By starting with a different register seed value, and running the sequence through the system, the estimated impulse response will have a slightly different output and the averaging of all of the estimated impulse responses will aid in the artifact rejection as demonstrated in (Sohrab et al., 1978).

The MATLAB function written in conjunction with this thesis has been included for reference:

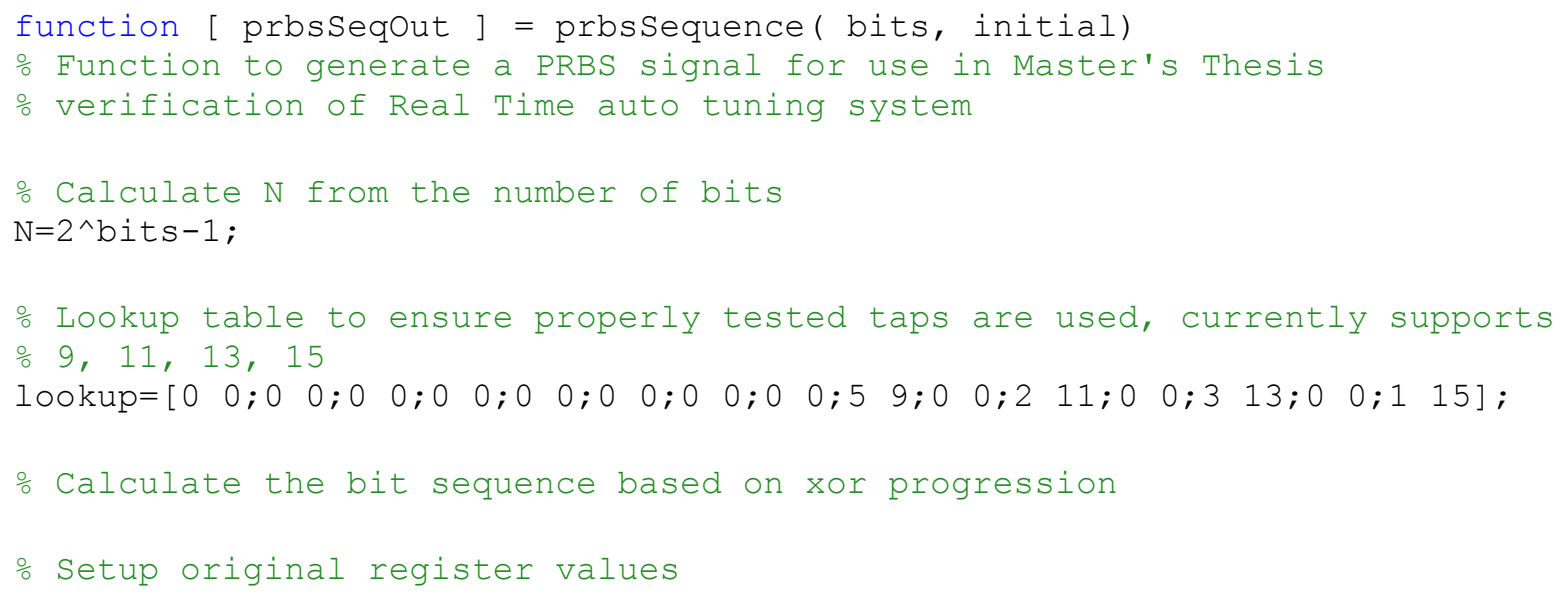




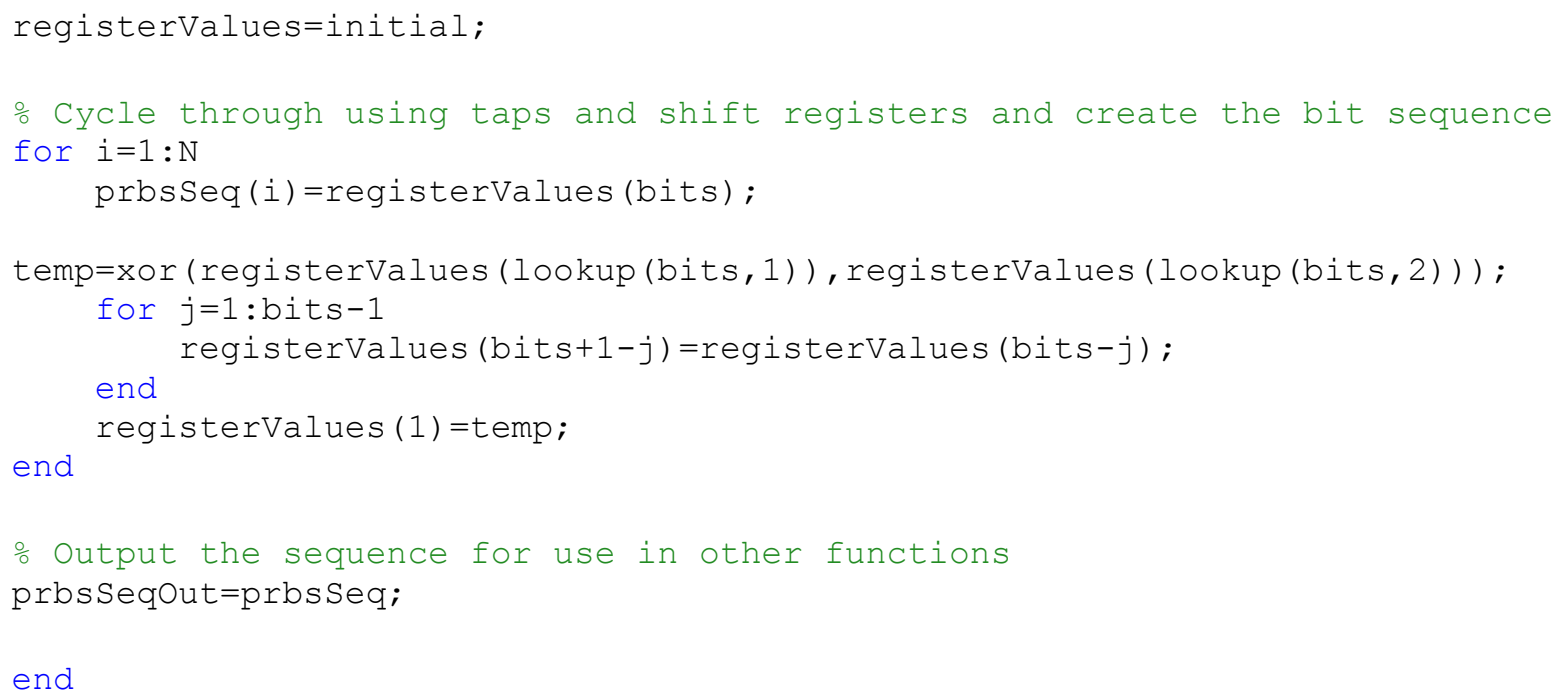




\section{Appendix B}

\section{System Identification Routine Using MATLAB}

There was a large amount of research that went into the application of the lsqcurvefit() function for the algorithm presented in this work. The method involved the creation of optimization functions for each type of plant and then the appropriate application of the lsqcurevefit() function to minimize the mean square error between the target function and the frequency response data. By proper utilization of these functions, an appropriate solution was found that worked for the cases tested. The MATLAB code for the application of the identification routine and the optimization functions has been included for reference:

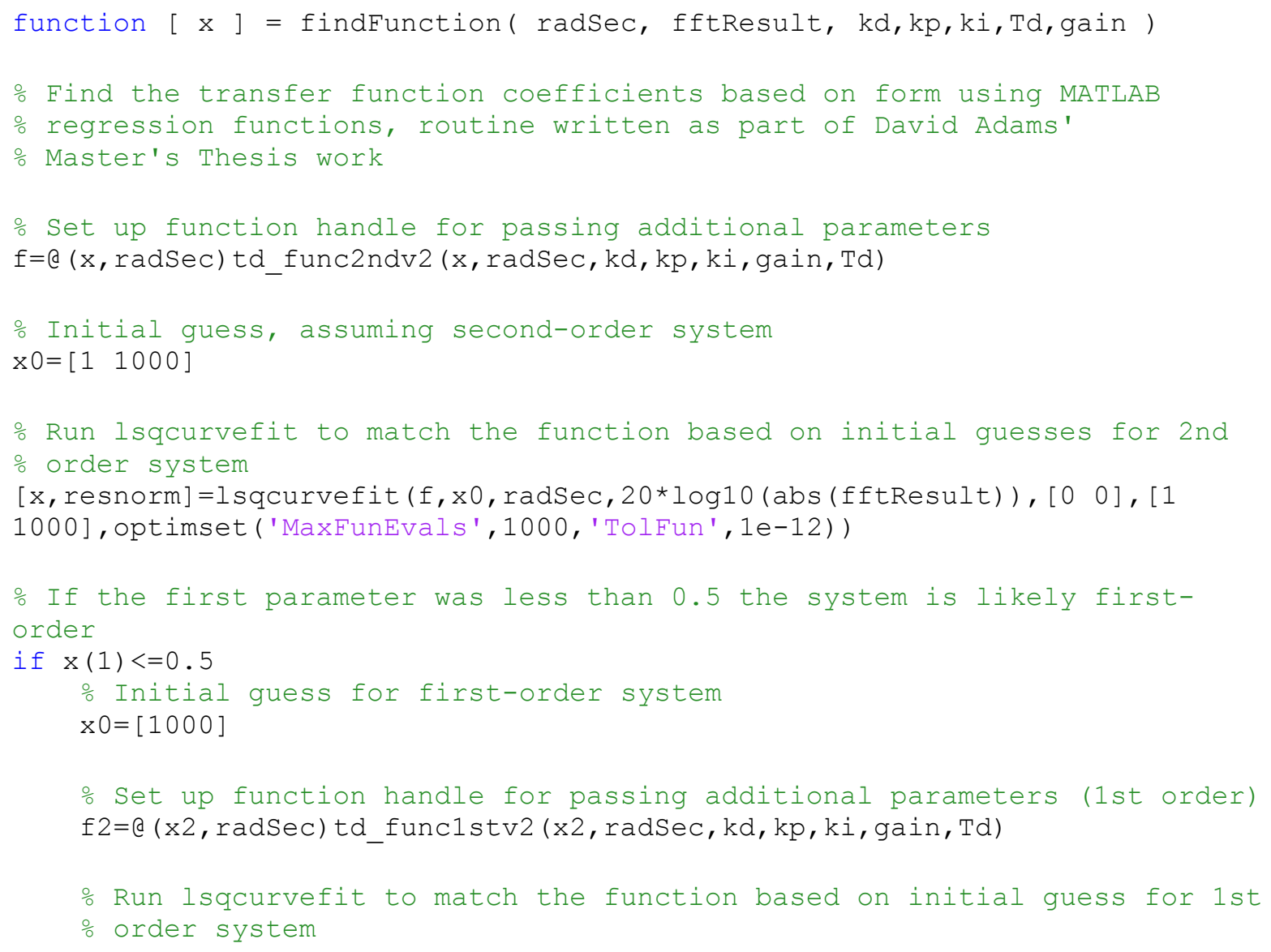


$[\mathrm{x} 2$, resnorm $]=$ lsqcurvefit $(\mathrm{f} 2, \mathrm{x} 0$, radSec, $20 * \log 10$ (abs (fftResult)), [0], [1000], optimset ('MaxFunEvals ',1000, 'TolFun', 1e-12))

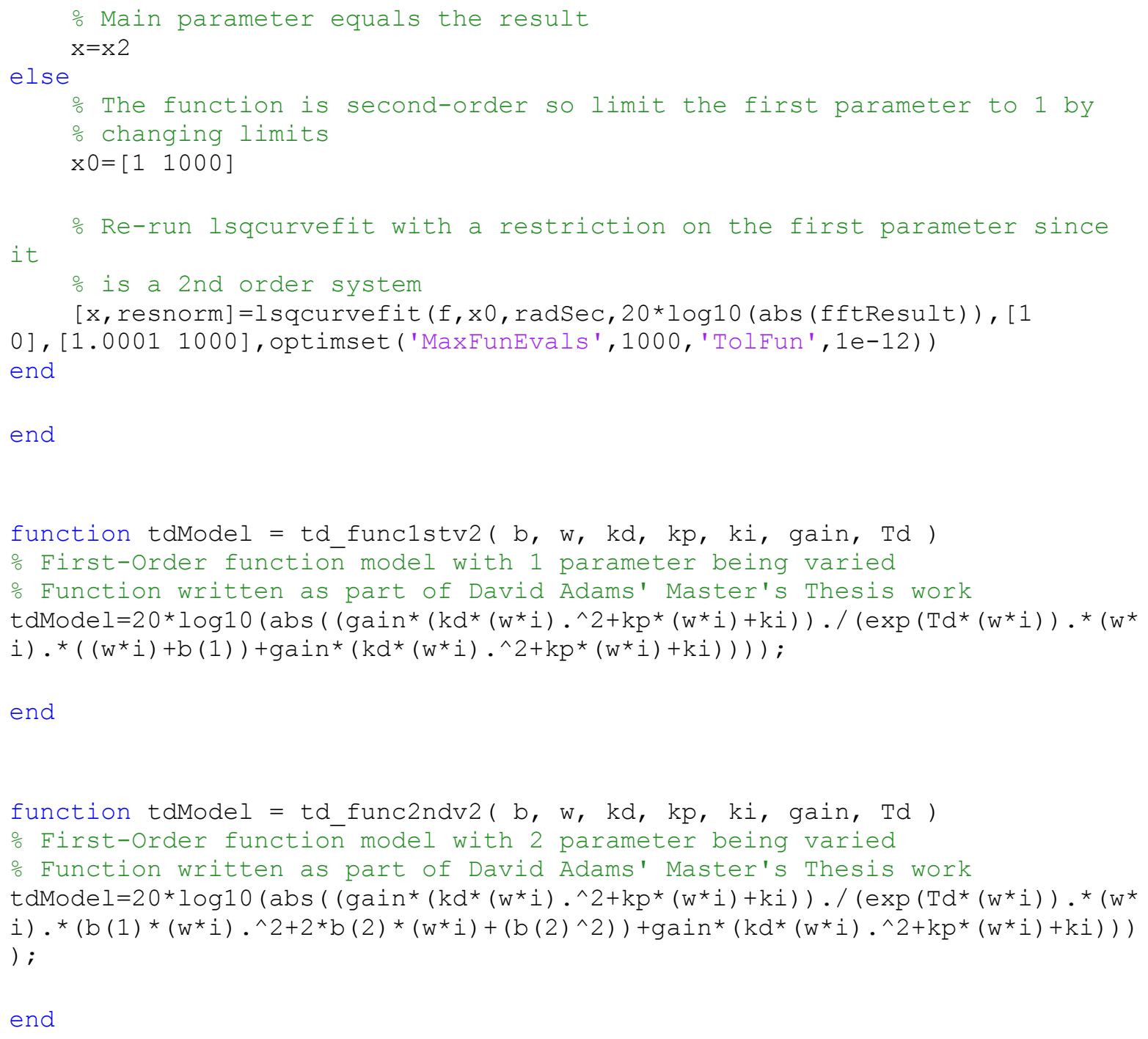




\section{Appendix C}

\section{Algorithm for Real-Time Auto Tuning With Time-Delay}

The actual algorithm that was run for the real-time auto tuning validation and testing ties together all of the sub topics contained in this thesis. This function assumes that the user has already collected the data from the system and is ready to pass that data to the auto tuning algorithm. This algorithm takes in the data, along with the known system parameters, and a commanded overshoot and returns the tuned PID values to place the compensator and the loop gain to achieve the commanded overshoot. This function computes sampling frequency and sample time to properly scale the FFT results and computes the frequency data needed for system identification. The algorithm then runs the system identification routine as discussed in Appendix B. Once the system is identified, the algorithm prints out the Bode Plots and calculates the compensator zero placement and the corresponding gain for the system. This result is then returned to be applied to the system to achieve a satisfactory response. The MATLAB algorithm written for this method has been included for reference and contains the gain calculation section near the end of the algorithm.

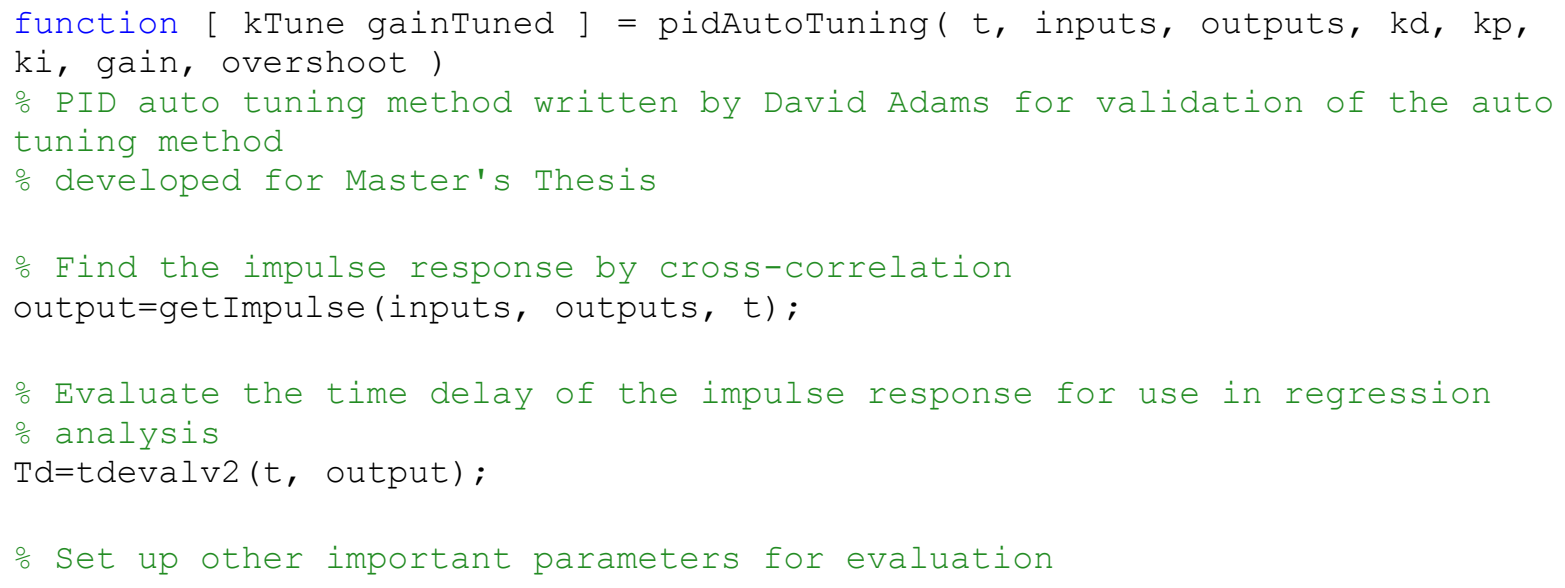




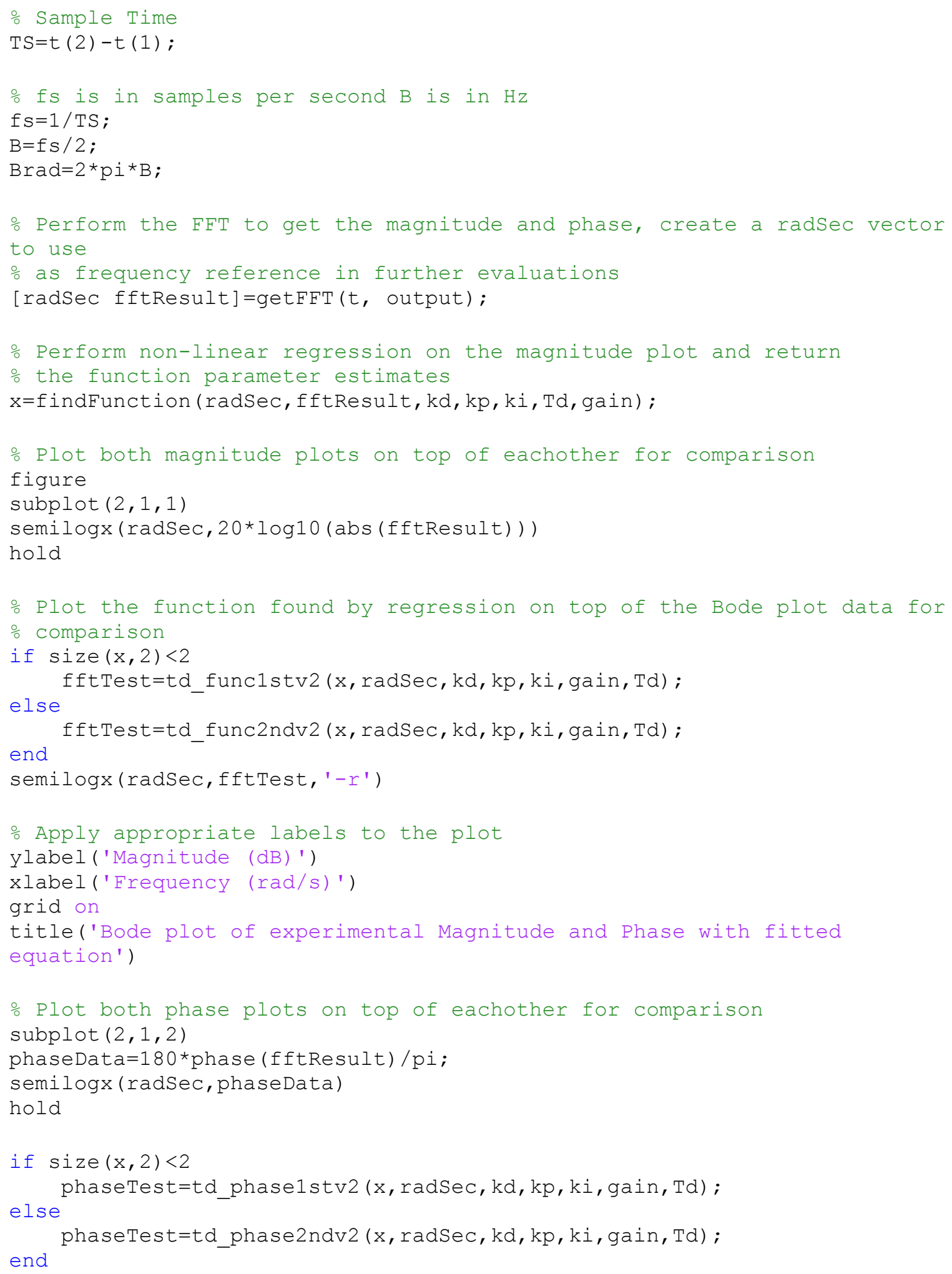




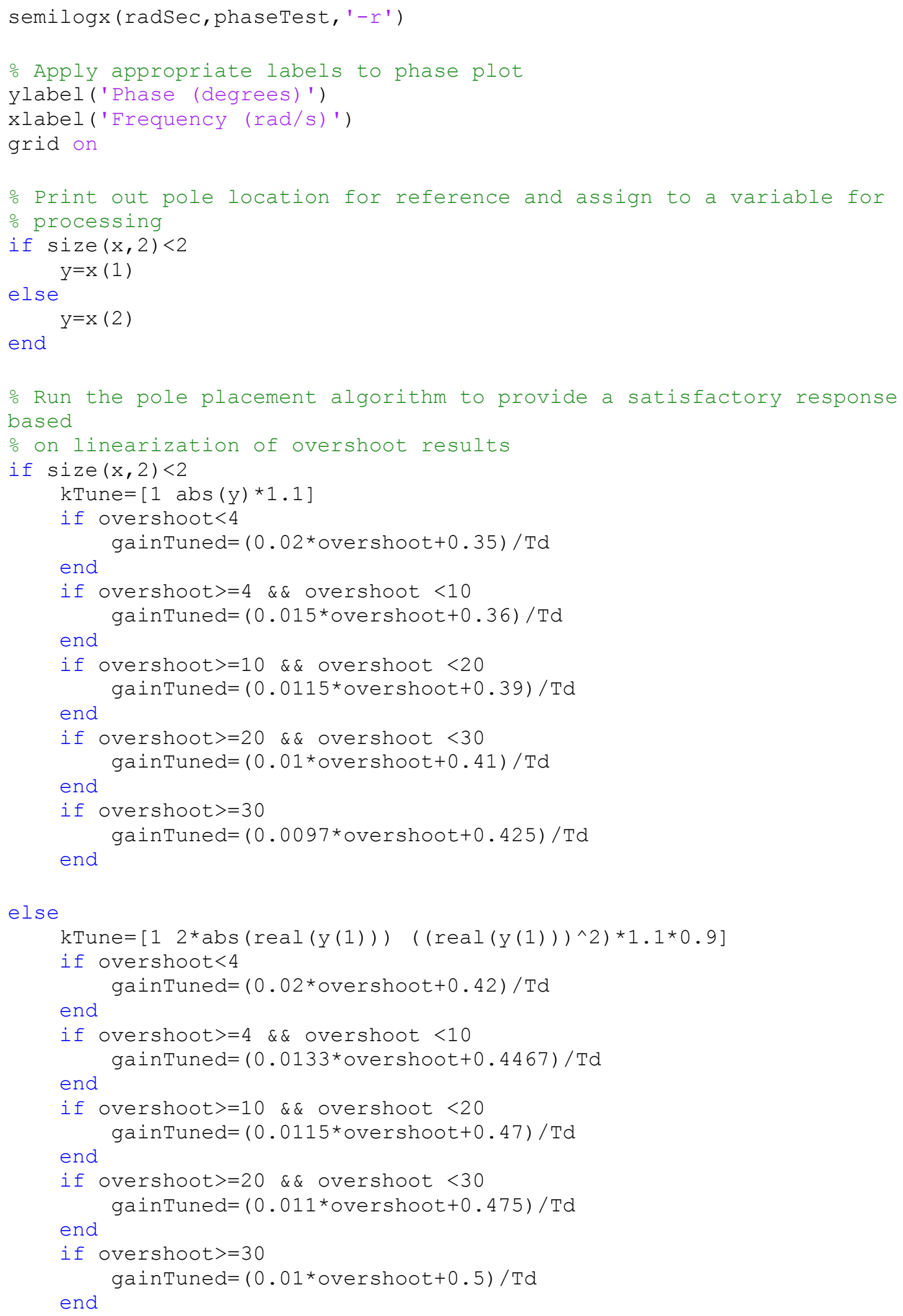


end

\% Function returns the recommended tuning parameters and the recommended \% gain found by the auto tuning algorithm

end 